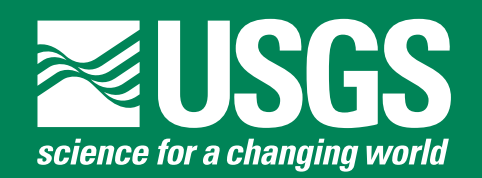

\title{
Migration of the Acadian Orogen and Foreland Basin Across the Northern Appalachians of Maine and Adjacent Areas
}

Professional Paper 1624 


\section{Availability of Publications of the U.S. Geological Survey}

Order U.S. Geological Survey (USGS) publications from the offices listed below. Detailed ordering instructions, along with prices of the last offerings, are given in the current-year issues of the catalog "New Publications of the U.S. Geological Survey."

\section{Books, Maps, and Other Publications}

\section{By Mail}

Books, maps, and other publications are available by mail from-

USGS Information Services

Box 25286, Federal Center

Denver, CO 80225

Publications include Professional Papers, Bulletins, WaterSupply Papers, Techniques of Water-Resources Investigations, Circulars, Fact Sheets, publications of general interest, single copies of permanent USGS catalogs, and topographic and thematic maps.

\section{Over the Counter}

Books, maps, and other publications of the U.S. Geological Survey are available over the counter at the following USGS Earth Science Information Centers (ESIC's), all of which are authorized agents of the Superintendent of Documents:

- Anchorage, Alaska-Rm. 101, 4230 University Dr.

- Denver, Colorado-Bldg. 810, Federal Center

- Menlo Park, California-Rm. 3128, Bldg. 3, 345 Middlefield Rd.

- Reston, Virginia-Rm. 1C402, USGS National Center, 12201 Sunrise Valley Dr.

- Salt Lake City, Utah-2222 West, 2300 South (books and maps available for inspection only)

- Spokane, Washington-Rm. 135, U.S. Post Office Building, 904 West Riverside Ave.

- Washington, D.C.-Rm. 2650, Main Interior Bldg., 18th and C Sts., NW.

Maps only may be purchased over the counter at the following USGS office:

- Rolla, Missouri-1400 Independence Rd.

\section{Electronically}

Some USGS publications, including the catalog "New Publications of the U.S. Geological Survey" are also available electronically on the USGS's World Wide Web home page at http://www.usgs.gov

\section{Preliminary Determination of Epicenters}

Subscriptions to the periodical "Preliminary Determination of Epicenters" can be obtained only from the Superintendent of
Documents. Check or money order must be payable to the Superintendent of Documents. Order by mail from-

Superintendent of Documents

Government Printing Office

Washington, DC 20402

\section{Information Periodicals}

Many Information Periodicals products are available through the systems or formats listed below:

\section{Printed Products}

Printed copies of the Minerals Yearbook and the Mineral Commodity Summaries can be ordered from the Superintendent of Documents, Government Printing Office (address above). Printed copies of Metal Industry Indicators and Mineral Industry Surveys can be ordered from the Center for Disease Control and Prevention, National Institute for Occupational Safety and Health, Pittsburgh Research Center, P.O. Box 18070, Pittsburgh, PA 15236-0070.

\section{Mines FaxBack: Return fax service}

1. Use the touch-tone handset attached to your fax machine's telephone jack. (ISDN [digital] telephones cannot be used with fax machines.)

2. Dial (703) 648-4999.

3. Listen to the menu options and punch in the number of your selection, using the touch-tone telephone.

4. After completing your selection, press the start button on your fax machine.

\section{CD-ROM}

A disc containing chapters of the Minerals Yearbook (199395), the Mineral Commodity Summaries (1995-97), a statistical compendium (1970-90), and other publications is updated three times a year and sold by the Superintendent of Documents, Government Printing Office (address above).

\section{World Wide Web}

Minerals information is available electronically at http://minerals.er.usgs.gov/minerals/

\section{Subscription to the catalog "New Publications of the U.S. Geological Survey"}

Those wishing to be placed on a free subscription list for the catalog "New Publications of the U.S. Geological Survey" should write to-

U.S. Geological Survey

903 National Center

Reston, VA 20192 


\section{Migration of the Acadian Orogen and Foreland Basin Across the Northern Appalachians of Maine and Adjacent Areas}

By Dwight C. Bradley, Robert D. Tucker, Daniel R. Lux, Anita G. Harris, and D. Colin McGregor

Professional Paper 1624 


\title{
U.S. Department of the Interior \\ Bruce Babbitt, Secretary
}

\author{
U.S. Geological Survey \\ Charles G. Groat, Director
}

Any use of trade, product, or firm names in this publication is for descriptive purposes only and does not imply endorsement by the U.S. Government.

United States Government Printing Office: 2000

For additional copies please contact:

\section{USGS Information Services}

Box 25286

Denver, CO 80225

For more information about the USGS and its products:

Telephone: 1-888-ASK-USGS

World Wide Web: http://www.usgs.gov/

Text edited by George A. Havach

Layout and design by Stephen L. Scott

Manuscript approved for publication, November 8, 1999

Library of Congress Cataloging-in-Publication Data

Migration of the Acadian Orogen and Foreland Basin across the Northern Appalachians of Maine and adjacent areas / Dwight C. Bradley... [et al.].

p. cm. -- (U.S. Geological Survey professional paper ; 1624) Includes bibliographical references.

1. Orogeny--Maine. 2. Geology, Stratigraphic--Silurian. 3. Geology, Stratigraphic--Devonian. 4. Geology, Structural--Maine. I. Bradley, Dwight Culver. II.

QE75.P9 v. 1624

[QE621.5.U]

$557.3 \mathrm{~s}-\mathrm{-dc} 21$

[551.7’32’09741] 


\section{CONTENTS}

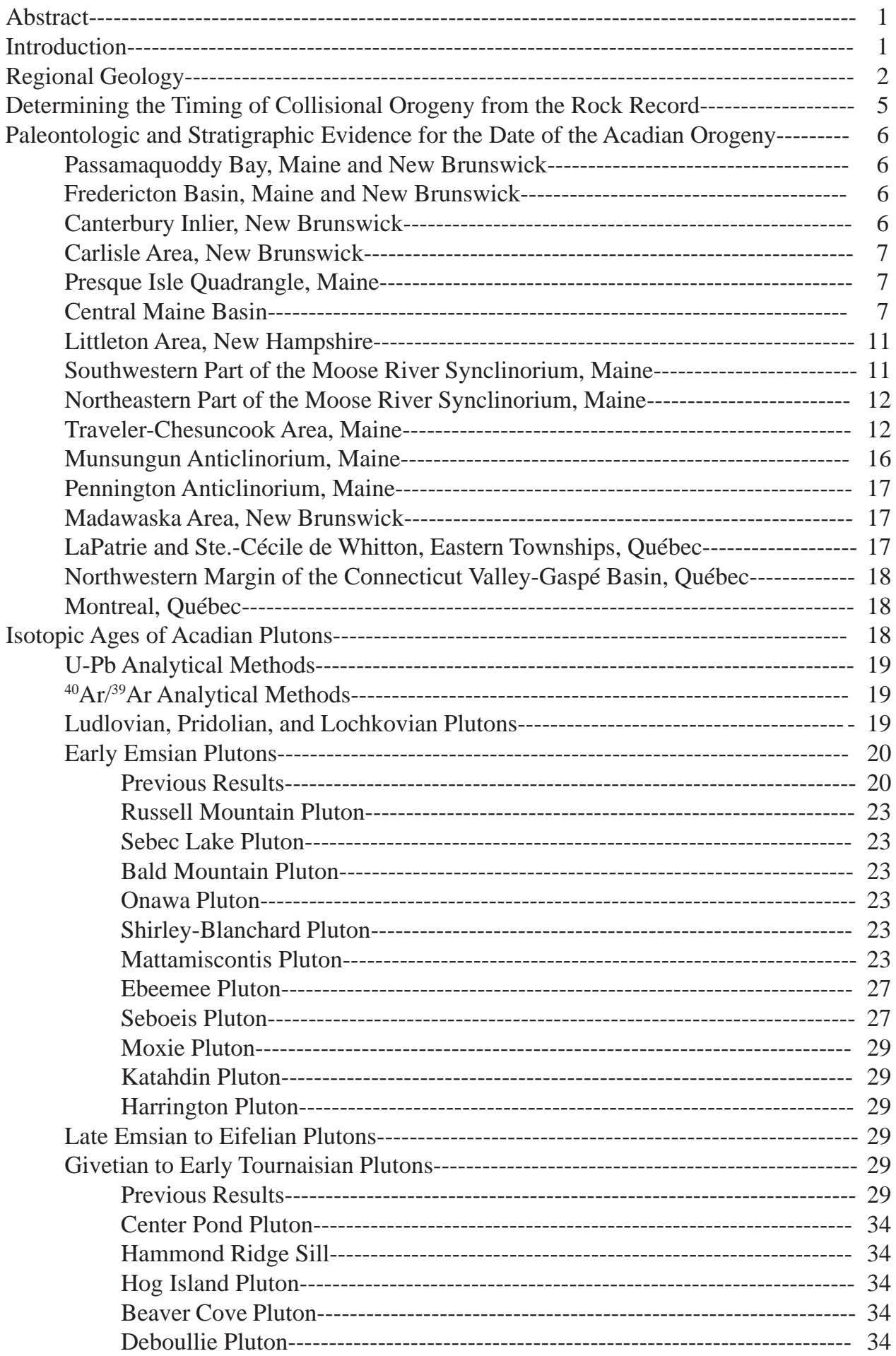




\section{CONTENTS}

Isotopic Ages of Acadian Plutons-Continued

Givetian to Early Tournaisian Plutons-Continued

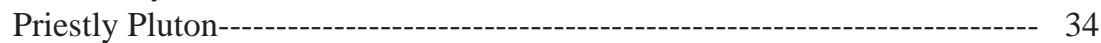

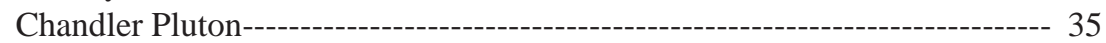

Seboeis Pluton------------------- 35

The Silurian-Devonian Time Scale: Basis for Correlations Between

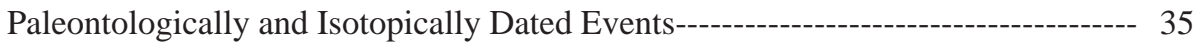

Location Through Time of the Acadian Deformation Front and Foreland Basin--------- 35

Palinspastic Considerations-----_-_-_-_- 36

Early Ludlow-------------------------------------------------------------------- 39

Early Lochkovian-------------------------------------------------------------- 40

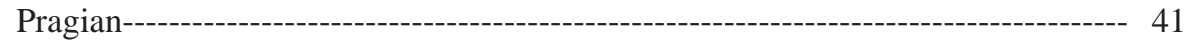

Early Emsian------------------------------------------------------------------------ 41

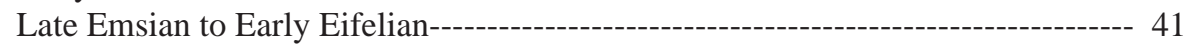

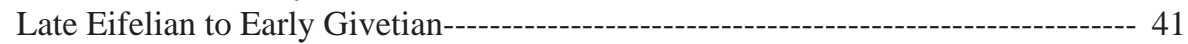

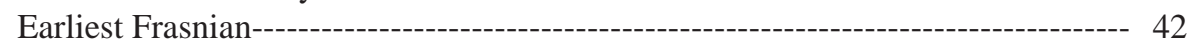

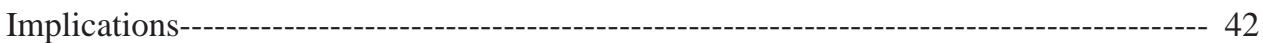

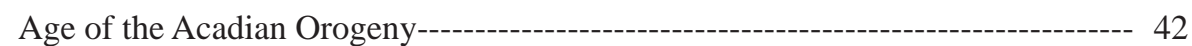

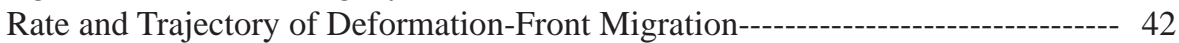

Physical Setting of Magmatism--------- 43

Coastal Volcanic Belt and the Ludlovian to Lochkovian Plutonic Belt------ 43

Piscataquis Volcanic Belt and the Emsian Plutonic Belt------------------ 43

Syncollisional Versus Precollisional Plate Geometry---------------------------- 43

Hinterland Deformational Regimes and Regional Partitioning of Acadian

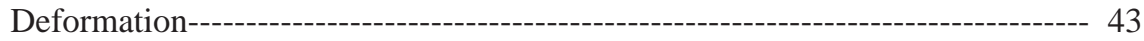

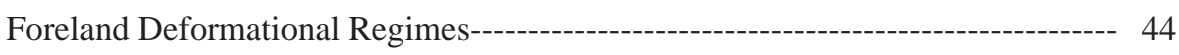

Suggestions for Further Work---

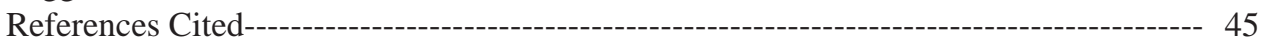

\section{PLATES}

[Plates follow references]

1. Photomicrographs of Late Silurian to early Middle Devonian conodonts from Maine and southern Québec,

Canada

2. Photomicrographs of Late Silurian to earliest Devonian conodonts from Maine

\section{FIGURES}

1. Geologic map of Maine and adjacent areas--

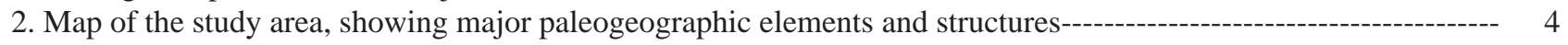

3. Cross-sectional diagram showing a conceptual model of two-plate collision--

4. Stratigraphic sections bearing on the age of Acadian deformation in the study area--

5. Correlation chart showing conodont and palynomorph age ranges for samples dated in this study-------------- 12

6. Map of Maine and adjacent areas, showing distribution of Silurian and Devonian plutons----------------------- 20

7. U-Pb concordia diagrams of igneous rocks---

8. ${ }^{40} \mathrm{Ar} /{ }^{\beta 9} \mathrm{Ar}$ age spectra of igneous rocks--- 22

9. Thin-section photomicrographs of contact-metamorphosed rocks adjacent to plutons-------------------------- 28

10. Map of the study area, showing successive positions of the Acadian deformation front--------------------- 36 
11. Map of the study area, showing successive positions of the leading edge of the Acadian foreland basin----------

12. Schematic, nonpalinspastic paleogeographic maps of the study area, showing migration of the orogen and foreland basin--

13. Map of the study area, showing local estimates of Acadian and younger shortening

\section{TABLES}

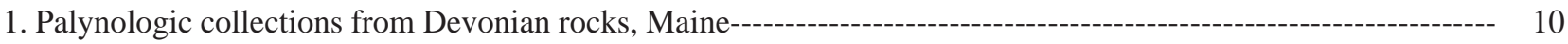

2. Conodont collections from Silurian and Devonian rocks, Maine and Québec------------- 13

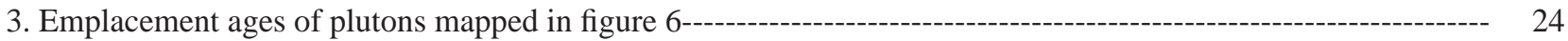

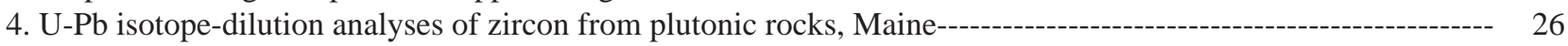

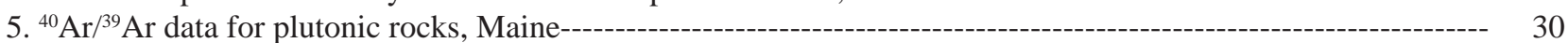

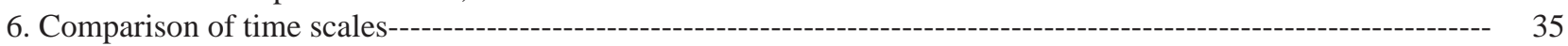

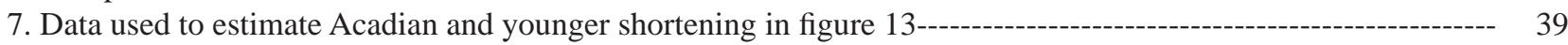




\title{
Migration of the Acadian Orogen and Foreland Basin Across the Northern Appalachians of Maine and Adjacent Areas
}

\author{
By Dwight C. Bradley, Robert D. Tucker, Daniel R. Lux, \\ Anita G. Harris, and D. Colin McGregor ${ }^{1}$
}

\section{Abstract}

We reconstruct seven sequential positions of the Acadian deformation front and foreland basin to illustrate the northwestward migration of the orogenic belt across Maine and adjacent areas from Late Silurian to Middle Devonian time. The reconstructions are based on (1) U-Pb and ${ }^{40} \mathrm{Ar} /{ }^{39} \mathrm{Ar}$ ages of pretectonic, syntectonic, and posttectonic plutons; (2) conodont and palynomorph ages of key strata that predate or postdate the local age of deformation; and (3) interpretations of the depositional settings of these strata: far foreland, foreland basin, inner trench slope, and orogenic hinterland. Tight correlations between isotopically and paleontologically dated events are made possible by recent improvements in the Silurian-Devonian time scale.

During early Ludlow time ( 423 Ma), the deformation front must have lain near the present midline of the Fredericton Basin, between the posttectonic Pocomoonshine pluton (423 Ma) to the southeast and the coeval graptolitic Smalls Falls Formation, $70 \mathrm{~km}$ across strike to the northwest. During early Lochkovian time ( 417 Ma), the deformation front was near the present midline of the Central Maine Basin, as recorded by the northwestward advance of slope deposits of the Carrabassett Formation across axially transported turbidites of the Madrid Formation. The early Emsian (407-406 Ma) deformation front lay along or near the Lobster anticlinorium; its position is bracketed between the posttectonic Russell Mountain pluton (406 Ma) to the southeast and the coeval brachiopod-bearing Tomhegan molasse, $50 \mathrm{~km}$ across strike to the northwest. At the Emsian-Eifelian boundary ( 394 Ma), the deformation front was located along the Pennington-Munsungun anticlinorium, northwest of the posttectonic Mapleton and Trout Valley Formations but southeast of the youngest nonmarine clastic rocks in the pretectonic Fish River Lake Formation. At the Eifelian-Givetian boundary ( 387.5 Ma), the deformation front was probably somewhere near the midline of the Connecticut Valley-Gaspé Basin, northwest of a belt of posttectonic plutons in Québec, including the 384-Ma Scotstown pluton, but southeast of three occurrences of deformed Eifelian carbonates along the basin's northwest-

${ }^{1}$ Geological Survey of Canada Contribution No. 1998178. ern margin. At the Givetian-Frasnian boundary ( 382.5 Ma), the deformation front was somewhere to the northwest of these Eifelian carbonate outcrops. The orogen thus migrated northwestward about $240 \mathrm{~km}$ across strike (present distance) in about 40.5 m.y. Meanwhile, on the outboard (southeasterly) side of the orogen, a boundary between deformed and undeformed rocks advanced southeastward at least $50 \mathrm{~km}$, probably during the Early and (or) Middle Devonian.

The migration pattern of the orogen and foreland basin suggests that during collision, a southeasterly plate that included the Acadian orogenic wedge and its Avalonian backstop overrode the Taconic-modified margin of North America. The implied minimum plate-convergence rate is about 6 $\mathrm{mm} / \mathrm{yr}$. The actual rate must have been considerably faster because this calculation was done on a nonpalinspastic base map; a more accurate estimate will have to await a careful assessment of Acadian shortening. If shortening reduced Maine to half its pre-Acadian width (a conservative estimate in light of the regional-scale tight to isoclinal folding), this situation would imply a plate-convergence rate of $12 \mathrm{~mm} / \mathrm{yr}$, at the slow end of the normal range of modern plate motions. The reconstructed positions of the orogen and foreland basin also constrain the setting of several Silurian and Devonian episodes of volcanism and plutonism, certain post-Acadian deformations in the Acadian orogenic hinterland, and certain pre-Acadian deformations in what was then the Acadian foreland.

\section{Introduction}

More than three decades after Wilson's (1966) first platetectonic interpretation of the Appalachians, a consensus on the plate geometry that led to the Acadian orogeny still has not emerged, though not for lack of trying. In this report, we present new results and review old evidence bearing on orogenic timing on a regional scale. By focusing on the syncollisional history, we can make new headway on the Acadian problem without getting bogged down in controversies regarding the precollisional plate geometry.

Donahoe and Pajari (1973) presented evidence that the Acadian orogeny was diachronous across strike, beginning in 
the Early Devonian along the Maine-New Brunswick coast and ending during the Middle Devonian in Québec, near the Gulf of the St. Lawrence. This finding was based on the age and distribution of fossiliferous strata known to predate and postdate deformation and on isotopic ages of posttectonic plutons. Five developments make it worthwhile to revisit this topic. First, the Silurian-Devonian time scale has undergone major revisions (Tucker and McKerrow, 1995; Tucker, and others, 1998). The new time scale is calibrated by U-Pb zircon ages of ash beds with good biostratigraphic control; most of the series and stage boundaries are 10 to $20 \mathrm{~m}$.y. older than on the time scale available to Donahoe and Pajari (1973), and the durations of individual series and stages differ widely as well. Second, many Acadian plutons have now been reliably dated by $\mathrm{U}-\mathrm{Pb}$ and ${ }^{40} \mathrm{Ar} /{ }^{39} \mathrm{Ar}$ methods. The new ages, with errors of 1 to 3 m.y., reveal that most of the Rb-Sr and conventional K-Ar ages used in Donohoe and Pajari's (1973) analysis were about as far off as the time scale. Third, certain key stratigraphic units that bear on the position of the deformation front over time have now been dated by using conodonts or spores with much greater precision and accuracy than was ever possible by using brachiopods, corals, and plants. Fourth, paleocurrent directions and depositional environments are now known for several key stratigraphic units, such as the Madrid and Carrabassett Formations (Hanson and Bradley, 1989, 1993; Bradley and Hanson, 1989); these data have helped us to locate the foreland basin during two time intervals - and, by inference, to locate the deformation front at the same times. Finally, as we will show, current understanding of orogenic wedges, flexural foreland basins, and orogenic backstops makes it possible to glean more information from conventional stratigraphic evidence about orogenic timing than was possible in the 1970's.

New U-Pb, ${ }^{40} \mathrm{Ar} /{ }^{39} \mathrm{Ar}$, conodont, and palynologic data, which were the basis for the regional tectonic conclusions by Bradley and others (1998) and Robinson and others (1998), are documented here. These new results, as well as previously published data, enable us to track seven sequential positions of the deformation front as the first wave of Acadian deformation migrated cratonward across the northern Appalachians during Late Silurian to Middle Devonian time. This information has implications for (1) the rates, trajectories, and amounts of plate convergence; (2) the syncollisional plate geometry; (3) strike-slip and thrust partitioning during collision; and (4) the relation between various suites of igneous rocks and the orogenic belt as it existed at the time of magmatism.

Acknowledgments.-Bradley and Harris were funded by the Gilbert Fellowship program of the U.S. Geological Survey; Tucker was funded by U.S. National Science Foundation grants EAR-9506693 and EAR-9304142. Reviews by Tom Armstrong and Allan Ludman substantially improved the manuscript. We thank Zhan Peng (Washington University), Dan Griffin (University of Maine, Orono), and Tim Plucinsky (U.S. Geological Survey) for help in their respective geochronology and mineral-separation labs, and Kevin Moore for help in the field. Many colleagues generously shared their knowledge and time, including Lindley Hanson (Salem State Col- lege), Dykstra Eusden (Bates College), Jim Hibbard (North Carolina State University), Allan Ludman (Queens College), Philip Osberg (University of Maine at Orono), Steve Pollock (University of Southern Maine), Peter Robinson (University of Massachusetts), David Roy (Boston College), Wally Bothner (University of New Hampshire), Charles ver Straeten (New York State Geological Survey), Tom Uyeno and Cees van Stall (Geological Survey of Canada), Kim Hannula (Middlebury College), Sandra Barr (Acadia University), and Michael Foose, Tom Armstrong, D.B. Stewart, Douglas Rankin, and Bob Moench (U.S. Geological Survey). Alison Till (U.S. Geological Survey) helped with interpretations of thin sections. None of this research would have been possible without the stratigraphic framework that largely rests on fossil identifications by Art Boucot and William Berry. Logistic support was provided by Dee Caldwell (Boston University), Bob Marvinney (Maine Geological Survey), Darrell Cowing (U.S. Geological Survey, Maine Water District office), and the Bradley family of Randolph, N.H.

\section{Regional Geology}

The study area is the Acadian-deformed belt of Maine, plus adjacent parts of Vermont, New Hampshire, the Eastern Townships of Québec, and New Brunswick. The regional geology was reviewed by Osberg and others (1989) and Robinson and others (1998). The area of figure 1 is subdivided into 10 map units, 7 of which are relevant to the timing of Acadian deformation. Middle Ordovician and older rocks are grouped together in figure 1 because they provide no useful constraints on the age of Acadian tectonism.

Deep-water Silurian strata define four basins: the Connecticut Valley-Gaspé Basin, the Aroostook-Matapedia Basin, the Fredericton Basin, and the Central Maine Basin (fig. 2), the last two of which have been identified by some workers (Bradley, 1983; Ludman and others, 1993) as the former site of an ocean whose closure resulted in the Acadian orogeny. Although this interpretation remains controversial, the nature of the depositional basement of these deep-water basins makes little difference for the present study of syncollisional tectonics.

Flanking the deep-water basins are four tracts where preSilurian rocks are now exposed. These belts-Avalonia, the Bronson Hill-Pennington anticlinorial belt, Miramichi, and the Taconic orogen (fig. 2) - either were undergoing active erosion during at least part of the Silurian or were the sites of shallow-water deposition. The shallow-water deposits include carbonate and locally derived siliciclastic rocks that range in age from Llandovery to Eifelian. One goal of our study was to obtain precise conodont ages from the youngest carbonate rocks in various key stratigraphic sections.

From Late Silurian to Middle Devonian time, much of the area of figure 1 was inundated by a thick succession of clastic rocks, which we regard as the fill of a migrating Acadian foreland basin (Bradley, 1983, 1987, 1997; Hanson and Bradley, 1989). The foreland-basin succession includes all of the Devonian flysch and Devonian molasse and part of 
the deep-water Silurian sequence. For want of sedimentologic studies, the Silurian deep-water deposits cannot yet be confidently subdivided into foreland-basin and pre-foreland-basin successions. A second focus of the present study was to obtain more tightly constrained fossil ages from key forelandbasin deposits. Palynology has proved to be most useful in this regard; several calcareous concretions from siliciclastic turbidites were processed for conodonts but were found to be barren.
Silurian and Devonian volcanic rocks occur in two broad belts (figs. 1, 2). Rocks of the Coastal volcanic belt were erupted into basement of the Avalonian terrane along the coast of Maine and New Brunswick. Farther north lies a second belt of Silurian-Devonian volcanic rocks that crop out along parts of the Bronson Hill-Pennington anticlinorial belt, the Aroostook-Matapedia Basin, the Miramichi anticlinorium, and the Connecticut Valley-Gaspé Basin. The northerly belt has been called the Piscataquis volcanic belt (or magmatic belt) in New

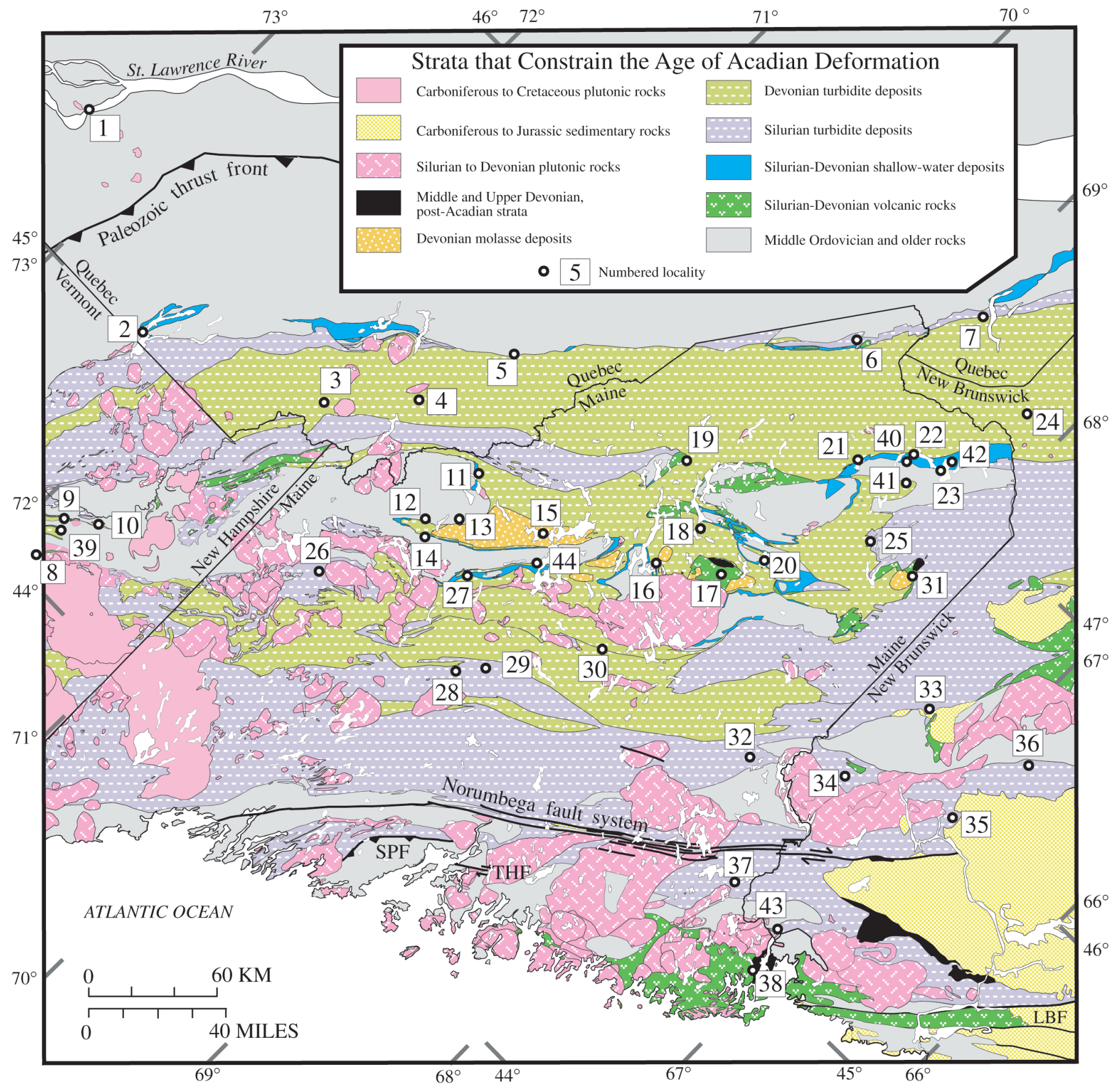

Figure 1.-Geologic map of Maine and adjacent parts of New Brunswick, Québec, New Hampshire, and Vermont, showing distribution of strata in study area that help constrain date of Acadian foreland-basin sedimentation and (or) Acadian deformation. Numbered localities are keyed to figure 4. LBF, Lubec-Belleisle Fault; SPF, Sennebec Pond Fault; THF, Turtle Head Fault. 
England (Bradley, 1983) and the Tobique volcanic belt in Canada (Keppie and Dostal, 1994). The Piscataquis volcanic rocks were erupted in a belt that had been accreted to North America during the Ordovician.

In all but the northernmost part of the study area (fig. 1), the rocks were deformed during a Silurian to Devonian orogeny that can be termed "Acadian" in the loose sense favored here. Where deformation was polyphase, the date of the first deformation event (D1) is of greatest interest for the present purposes. The style and intensity of Acadian deformation vary considerably across the study area; the common thread is that the deformation was dominantly contractional. In the low-grade rocks of northern and central Maine, Acadian structures are mostly upright, tight to isoclinal folds. At higher metamorphic grades in central New Hampshire, east-directed fold nappes predominate (Eusden and others, 1996); in southern New Hampshire, nappes have westerly vergence (Thompson and others, 1968; Robinson and others, 1998). Some strike-slip faulting also occurred during Late Silurian and Devonian times, as discussed below.

Silurian and Devonian (Acadian) plutons, ranging in composition from gabbro to granite, are widespread in the study area (fig. 1); many are demonstrably syntectonic or posttectonic. A major goal of our study was to date key plutons across a broad swath from the Maine coast to the Maine-Québec border, using U-Pb and ${ }^{40} \mathrm{Ar} /{ }^{39} \mathrm{Ar}$ methods. As we will show, the locus of plutonism shifted over time.

Of the various post-Acadian rocks in the study area (fig. 1), only Middle and Upper Devonian sedimentary and volcanic rocks that overlie Acadian-deformed rocks are relevant to

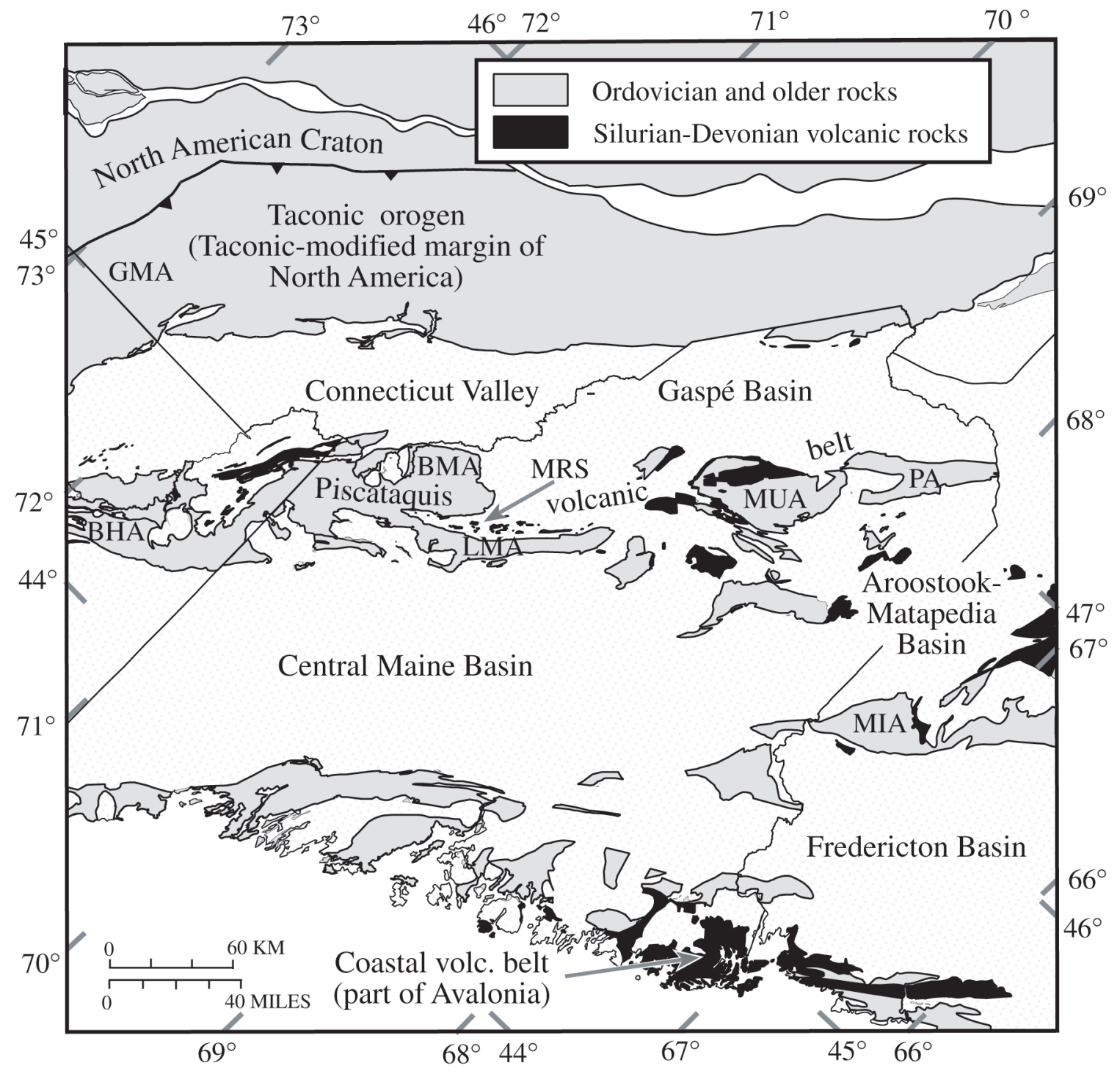

Figure 2.-Maine and adjacent parts of New Brunswick, Québec, New Hampshire, and Vermont, showing distribution of major Silurian-Devonian paleogeographic elements in study area. BHA, Bronson Hill anticlinorium; BMA, Boundary Mountains anticlinorium; GMA, Green Mountain-Sutton Mountain anticlinorium; LMA, Lobster Mountain anticlinorium; MIA, Miramichi anticlinorium; MRS, Moose River synclinorium; MUA, Munsungun anticlinorium; PA, Pennington anticlinorium. 
orogenic timing. In this report, we present new palynologic ages for two key post-Acadian units, ages that previously had been documented only in unpublished paleontologic reports of the Geological Survey of Canada. Other post-Acadian rocks in the study area include Carboniferous to Cretaceous plutons and Carboniferous to Jurassic sedimentary rocks.

\section{Determining the Timing of Collisional Orogeny from the Rock Record}

Traditionally, orogenesis in a given place has been judged to be younger than the youngest deformed strata but older than the oldest unit that unconformably overlies the deformed rocks. For most formations in the study area (fig. 1), we know more than whether they participated in Acadian deformation: interpretations of the tectonic setting of the dated rocks can supplement more traditional evidence for orogenic timing.

Typical contractional orogenic systems have three components: (1) an underriding plate, (2) an overriding plate, and, between them, (3) an actively deforming orogenic wedge (fig. 3). Two broad depositional regimes exist side by side on the underriding plate. The foreland basin is a marine or nonmarine sedimentary basin that flanks the orogen and is typically filled with orogenically derived sediment. (The terms "foreland basin" and "foredeep" are sometimes used interchangeably, but a foredeep, more properly, is an underfilled foreland basin characterized by deep-marine flysch sedimentation.) The term "far foreland" is coined here for the
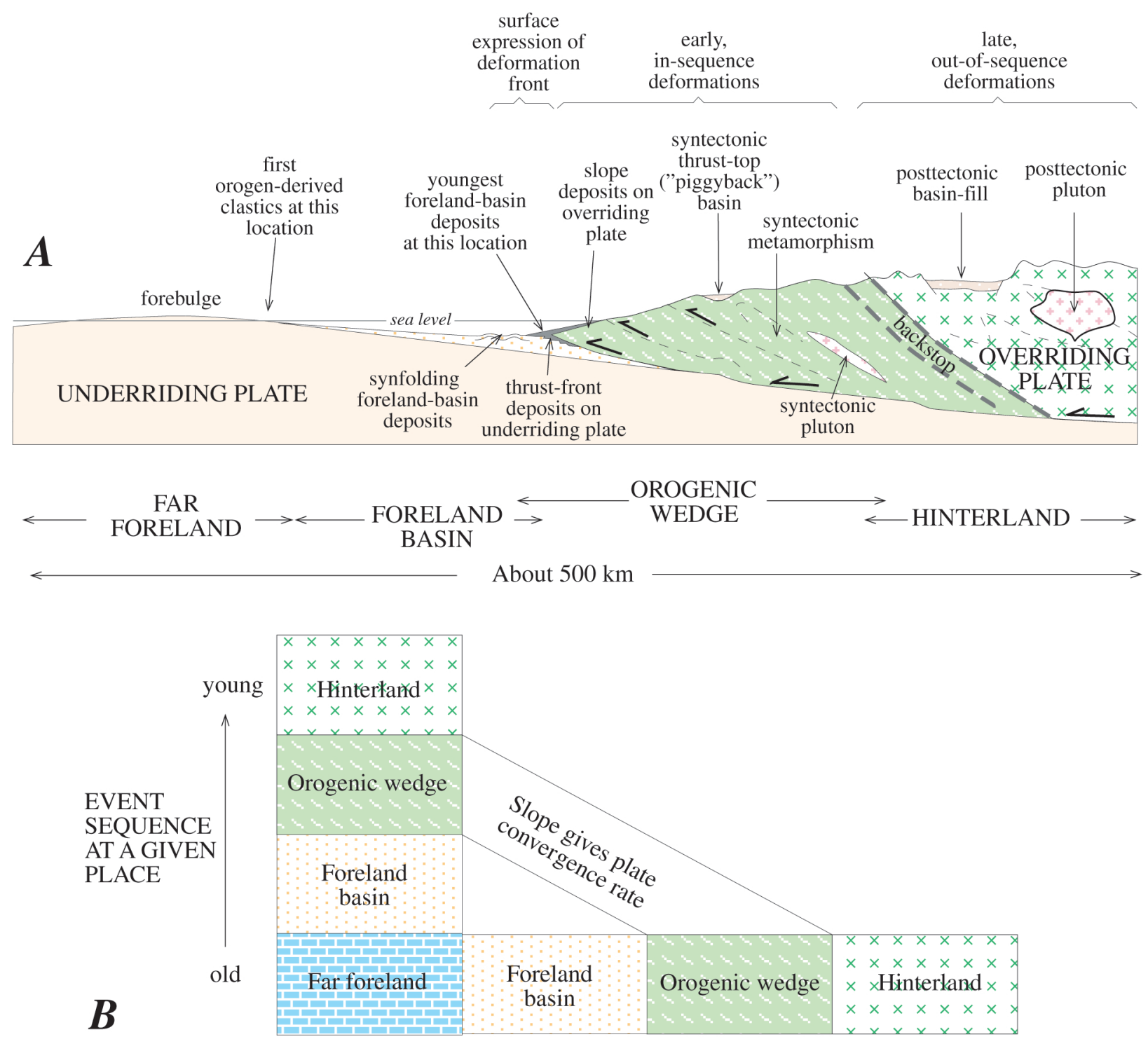

ACROSS-STRIKE DISTRIBUTION AT A GIVEN TIME

Figure 3.-Conceptual model of a two-plate collision. A, Cross section showing positions of far foreland, foreland basin, orogenic wedge, and hinterland, as discussed in text. B, Plate convergence causes each of these paleogeographic elements to migrate to left over time at a rate similar to plate-convergence rate, giving rise to succession of depositional and deformational events in a given rock sequence. 
region beyond the limit of orogen-derived sedimentation. A forebulge - a broad, gentle uplift formed by lithospheric flexure-may or may not be present in the distal part of a foreland basin or in the far foreland.

The deformation front is the surface expression of the gently dipping boundary between the underriding plate and orogenic wedge; it may be a sharp boundary at an emergent thrust fault or a broad zone of folding. Much, but not all, of the convergence between the underriding and overriding plates typically occurs at and just to the rear of the deformation front and is manifested as the D1 event that is of greatest interest here. Some additional convergence may be taken up farther to the rear, within the orogenic wedge, accounting for some D2 and later events. The boundary between the actively deforming orogenic wedge and the relatively rigid-or even extensional-hinterland on the overriding plate is called the backstop.

The model just outlined is sufficiently generalized that it applies to several plate-tectonic regimes, such as arc-passive margin collision, Andean-style foreland contraction, and arcarc collision. Figure 3 might be thought of as a single frame of a moving picture; as time goes on, new rocks of the underriding plate are fed into the orogenic system.

Most of the relevant Silurian-Devonian strata in the Acadian orogen can be placed with at least fair confidence in figure 3. In the descriptions of stratigraphic sections that follow, we focus not only on the conventional stratigraphic evidence (preorogenic versus postorogenic) but also on observations that enable us to place each section in the far foreland, foreland basin, orogenic wedge, or hinterland at a particular time. We use only those age constraints provided by fossils where they actually occur; the New England tradition of extrapolating fossil ages across strike hinders the recognition of diachronous facies, which are characteristic of all collisional orogens in the world.

\section{Paleontologic and Stratigraphic Evidence for the Date of the Acadian Orogeny}

\section{Passamaquoddy Bay, Maine and New Brunswick}

The Coastal volcanic belt section of southeastern Maine (loc. 38, figs. 1, 4) and southwestern New Brunswick consists of $8 \mathrm{~km}$ of interbedded bimodal volcanic and fossiliferous sedimentary rocks of late Llandovery to Lochkovian age. The following highlights (from Gates and Moench, 1981) are key to Acadian tectonic problems. The late Llandovery Quoddy Formation, mostly graptolitic shale, contains a few tuffaceous horizons that record the first known pulse of Silurian volcanism in the belt (loc. 38, fig. 4). Mixed volcanism and ever-shallowing marine sedimentation continued throughout Wenlock, Ludlow, and Pridoli time and the first part of the Lochkovian (Dennys, Edmunds, Leighton, Hershey, and lower Eastport Formations). In New Brunswick, the upper part of the Eastport Formation consists of fluvial sandstone, siltstone, and conglomerate (Pickerill and others, 1978). The Eastport Formation, which is the youngest pre-Acadian stratified unit, has been assigned a Lochkovian age on the basis of a restricted fauna of lingulids, gastropods, pelecypods, and ostracodes (Berdan, 1971; Pickerill and Pajari, 1976). We note that the Castine Volcanics in the Penobscot Bay region, which were once regarded as Silurian and thus part of the Coastal volcanic belt, have recently yielded a U-Pb zircon age of $505 \mathrm{Ma}$ (R.D. Tucker, unpub. data., 1997) and so are irrelevant to the Acadian orogeny. All the volcanic units were involved in Acadian folding and development of a regional east-northeast-striking cleavage (Pickerill and others, 1978, p. 42). The Red Beach pluton has yielded two conflicting isotopic ages, both suspect: a Givetian $\mathrm{Rb}-\mathrm{Sr}$ isochron age of 385 $\pm 6 \mathrm{Ma}$ (Spooner and Fairbairn, 1970; age recalculated herein) and a Lochkovian U-Pb age of $415 \pm 6 \mathrm{Ma}$ (Jurinski, 1990). The nominal U-Pb age is likely too old because the pluton truncates regional-scale Acadian folds that deform the Lochkovian Eastport Formation (Abbott, 1991); however, $\mathrm{Rb}-\mathrm{Sr}$ ages from coastal Maine plutons have consistently proved to be too young. Accordingly, the age of folding cannot be determined with confidence. The Perry Formation unconformably overlies both the Eastport Formation and the Red Beach pluton. The basal conglomerate of the Perry Formation contains boulders of unmistakable Red Beach granite. Plant fossils suggest a general Late Devonian age for the Perry Formation (Kasper and others, 1988, p. 127); an ash bed in the Perry Formation was processed for datable zircons but was barren.

\section{Fredericton Basin, Maine and New Brunswick}

In easternmost Maine, the thick, isoclinally folded turbidites of the Fredericton Basin (or "Trough" of some workers) are devoid of fossils (loc. 37, figs. 1, 4; Ludman and others, 1993). Nonetheless, some useful information regarding the timing of Acadian deformation can be gleaned. The youngest turbidite unit, the Flume Ridge Formation of presumed Silurian age (Ludman and others, 1993), was penetratively deformed before being intruded by the $423 \pm 2-\mathrm{Ma}$ Pocomoonshine pluton (see below). In New Brunswick, however, graptolites from turbidites (loc. 35, figs. 1, 4) reveal that sedimentation was still taking place in the northwestern part of the basin at this time. Specifically, the Burtts Corners Beds have yielded graptolites ranging in age from Cyrtograptus linnarssoni Zone (Wenlock) to Monograptus nilsonni Zone (early Ludlow) (Fyffe, 1995, p. 352-353).

\section{Canterbury Inlier, New Brunswick}

The Canterbury inlier (loc. 34, figs. 1, 4) lies along the southeast flank of the Miramichi anticlinorium. The inlier is separated by the Pokiok batholith from the main part of the Fredericton Basin, and the stratigraphy differs markedly. The Silurian-Devonian section is poorly fossiliferous, but 
the youngest stratified unit (Hartin Formation), which is of greatest interest here, is dated reasonably well. The Hartin Formation is composed of sandstone and slate, plus minor limestone, conglomerate, and felsic volcanic rocks. A brachiopod fauna indicates a Helderbergian age (that is, Lochkovian; A.J. Boucot, in Venugopal, 1979, p. 17). The Hartin Formation has been contact-metamorphosed by the Harkshaw and Skiff Lake phases of the Pokiok batholith (Venugopal, 1979, p. 28), which have yielded U-Pb ages of $411 \pm 2 \mathrm{Ma}$ (sphene) and 409 $\pm 2 \mathrm{Ma}$ (zircon), respectively (Bevier and Whalen, 1990a). Along strike in easternmost Maine, the Skiff Lake phase truncates Acadian structures that deform Silurian-Devonian strata (Ludman, 1990), and so appears to be broadly posttectonic. Acadian deformation in this area must therefore have occurred during the late Lochkovian or the Pragian. Given that Acadian deformation must have followed closely upon deposition of the Hartin Formation, a foreland-basin depositional setting seems likely.

\section{Carlisle Area, New Brunswick}

Acadian deformation is anomalously young in the Aroostook-Matapedia Basin in western New Brunswick (loc. 33, figs. 1, 4). The Late Ordovician and Silurian were times of deep-water sedimentation (Carys Mills and Smyrna Mills Formations; St. Peter, 1982). The Smyrna Mills Formation is overlain by the Costigan Mountain Formation of predominantly felsic volcanic rocks; it is undated but regarded as probably Lower Devonian on the basis of correlations with fossiliferous rocks to the northeast (St. Peter, 1982, p. 35). The youngest pre-Acadian unit is the Wapske Formation, estimated to be $4 \mathrm{~km}$ thick. It consists of slate, siltstone, sandstone, and conglomerate, with isolated mafic volcanic lenses (St. Peter, 1982). Clasts in conglomerate of the Wapske Formation can be traced to the Smyrna Mills, Costigan Mountain, and other older units (St. Peter, 1982, p. 40). The lower part of the Wapske Formation has yielded brachiopods from five localities that, according to A.J. Boucot (in St. Peter, 1982, p. 45), indicate a late Helderbergian (Becraft-Port Ewen) age. This interval corresponds approximately to that of the delta and pesavis conodont zones of the Lochkovian. Spores of late Emsian or early Eifelian age have been recovered from the upper, nonmarine part of the formation (D.C. McGregor, in St. Peter, 1982, p. 478). We suggest that the Wapske Formation was deposited in a foreland-basin setting and that it was derived, at least in part, from an Acadian orogenic source to the south, which must have existed by about $411 \mathrm{Ma}$.

\section{Presque Isle Quadrangle, Maine}

The Presque Isle-Mapleton section (loc. 31, figs. 1, 4) provides age constraints on the timing of Acadian foreland-basin sedimentation, the main Acadian folding, and postorogenic hinterland sedimentation. Deep-water sedimentation (slate, deep-water limestone, siliciclastic turbidites) was already underway by the Caradoc and continued into the Ludlow (Carys Mills and Spragueville Formations and Perham Group; Roy and Mencher, 1976). A gap in the megafossil record, spanning the late Ludlow, Pridoli, and early Lochkovian, corresponds to the "Salinic Disturbance" of Boucot and others (1964). Whatever its cause, this hiatus did not last as long as once suspected-no more than 6 m.y., judging from the new time scale (Tucker and others, 1998). The next rocks to be deposited were volcanic rocks (Dockendorff Group), deltaic sandstone (Chapman Sandstone), and prodeltaic turbidites (Swanback Formation). Boucot and others (1964) showed these three units to be lateral equivalents of Lochkovian ("New Scotland") age. Roy (1980) interpreted the Swanback and Chapman Formations as easterly derived flysch and molasse, respectively, which we assign to the Acadian foreland basin. Regional Acadian folding took place some time after deposition of the Lochkovian strata. The folded rocks are unconformably overlain by the Mapleton Formation, a local accumulation of nonmarine conglomerate and sandstone (Boucot and others, 1964). Conglomerate clasts in the Mapleton include fossiliferous clasts from the Chapman Sandstone and various older units (Boucot and others, 1964, p. 61-62; Roy and White, 1987). On the basis of plant fossils, the Mapleton was previously assigned a Middle Devonian, possibly Givetian age (Kasper and others, 1988). We now assign the Mapleton a slightly older age, early or middle Eifelian (Grandispora velatus-Rhabdosporites langii Zone), on the basis of spores from material submitted by Eli Mencher to D.C. McGregor during the early 1960's (table 1; McGregor, 1962; new slides examined, 1989). The following forms were identified: Apiculiretusispora gaspiensis, Ancyrospora sp., Calamospora cf. C. atava (Naumova) McGregor, Calyptosporites sp., Emphanisporites annulatus, E. rotatus, Grandispora velata, Stenozonotriletes sp., Dibolisporites echinaceus, and Acinosporites macrospinosus?. The Mapleton is folded into a gentle syncline of middle Eifelian or younger age, which is nearly coaxial with an older, tighter syncline of the main phase of Acadian deformation (Boucot and others, 1964, p. 73).

\section{Central Maine Basin}

The stratigraphy of the Central Maine Basin is best known from its northwest flank in the Rangeley and Phillips quadrangles (loc. 26, figs. 1, 4; Moench, 1971), where the Silurian-Devonian deep-water section is about $8 \mathrm{~km}$ thick and most of the formations have their type localities (Moench and Pankiwskyj, 1988). Key sedimentary-facies interpretations and paleocurrent data for these units, however, are from $150 \mathrm{~km}$ to the east in the Jo Mary Mountain area (loc. 30, fig. 1; Hanson and Bradley, 1989), where the rocks are at much lower metamorphic grade and sedimentary features are better preserved. Complicating matters still further, the fossil control that is critical for present purposes is from yet a third area, Kingsbury-Guilford (locs. 28, 29, figs. 1, 4), which before Acadian shortening lay many tens of kilometers from the basin's northwestern margin. The following discussion draws on information from all of these places. 
Along the northwest flank of the basin, the SilurianDevonian section is divisible into lower and upper parts on the basis of provenance and paleocurrents (Hanson and Bradley, 1993). Rocks assigned to the lower sequence (Greenvale Cove, Rangeley, Perry Mountain, and Smalls Falls Formations; see fig. 4) are known or inferred to have been derived from the northwest. A northwesterly provenance is well established for the Llandovery Rangeley Formation (at loc. 26, figs. 1,4), which contains conglomerate clasts from the Attean pluton some $50 \mathrm{~km}$ to the northwest (near loc. 12, fig. 1; Moench and Pankiwskyj, 1988). Meager paleocurrent data suggest that the Smalls Falls is the youngest unit of northwest derivation (Hanson and Bradley, 1993). It consists of rustyweathering turbiditic sandstone; the type Smalls Falls Formation is assigned a Ludlow age on the basis of fossils in broadly similar strata from a more southeasterly strike belt, as discussed below. The upper sequence, which was derived from outboard sources, consists of two widespread units-the Madrid and Carrabassett Formations-with poorly understood younger strata of local distribution. The Madrid Formation, in central Maine, is a thick succession of sandstone-dominated, siliciclastic turbidites; it becomes increas-

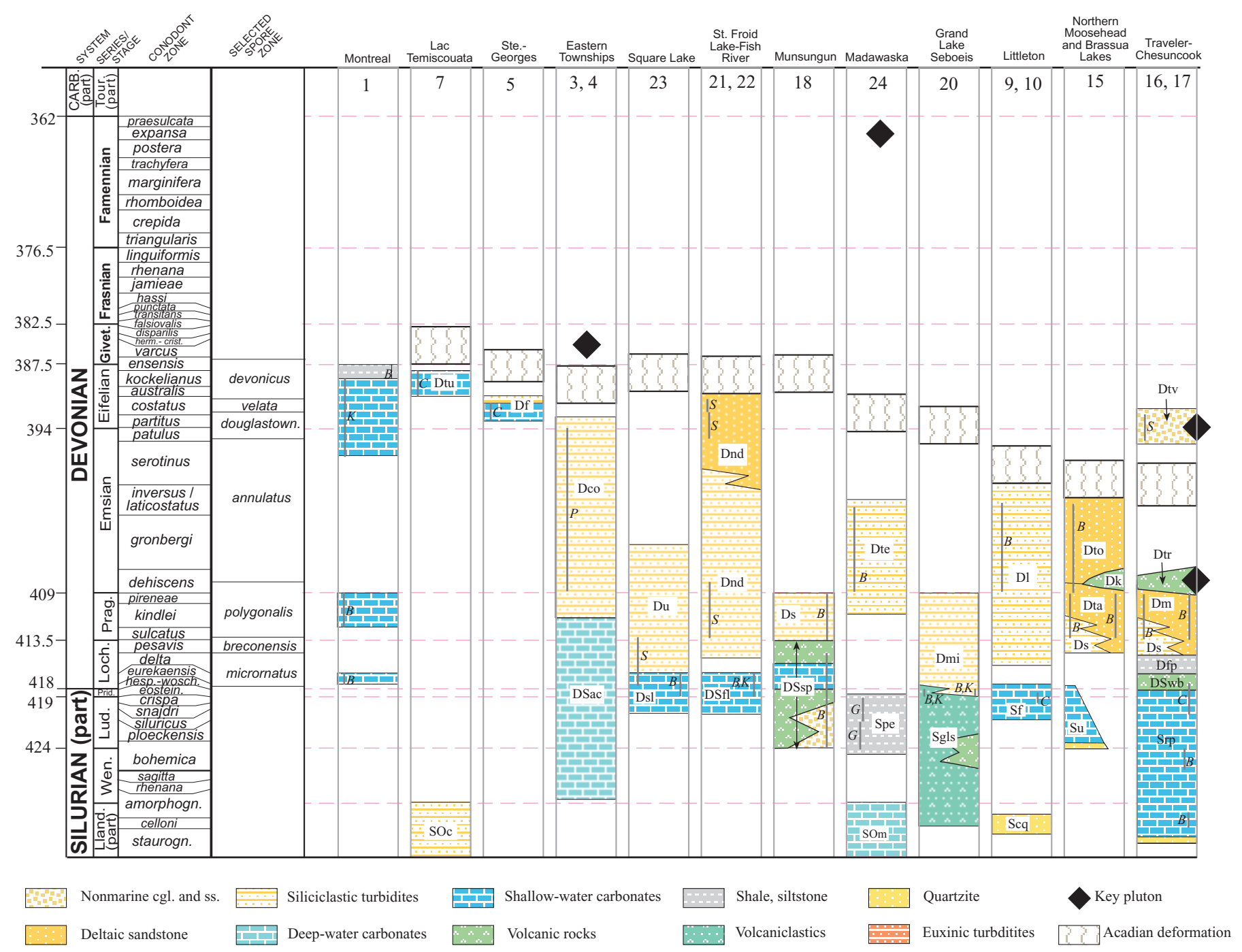

Figure 4.- Stratigraphic sections bearing on date of Acadian deformation and (or) foreland-basin sedimentation in study area (fig. 1). Numerical ages of various stage boundaries from time scale of Tucker and others (1998); spore zones from Richardson and McGregor (1986). Units: Dbk, Beck Pond Limestone; Dbr, Bear Pond Limestone Member; Dc, Carrabassett Formation; Dch, Chapman Sandstone; Dco, Compton Formation; De, Eastport Formation; Deh, Edmunds Hill Andesite; Df, Famine Limestone; Dfp, Frost Pond Formation; Dh, Hildreths Formation; Dha, Hartin Formation; Dhr, Hersey Formation; Dhv, Hedgehog Volcanics; Dk, Kineo Rhyolite; DI, Littleton Formation; Dm, Matagamon Sandstone; Dmc, McKenney Ponds Limestone Member; Dmi, Millimagassett Lake Formation; Dmp, Mapleton Formation; Dnd, Nadeau Thoroughfare beds (informal); Dpe, Perry Formation; Ds, Seboomook Group (formerly Formation); DSac, Ayres Cliff Formation; DScm, Costigan Mountain Formation; DSfl, lower part of the Fish River Lake Formation; DShl, Hersey and Leighton Formations; Dsl, Square Lake Limestone; DSm, Madrid Formation; DSpb, Parker Bog Formation; DSsp, Spider Lake Formation; Dsw, Swanback Formation; DSwb, West Branch Volcanics; Dta, Tarratine Formation; Dte, Temiscouata Formation; Dtf, The Forks Formation; Dto, Tomhegan Formation; Dtr, Traveler Rhyolite; Dtu, Touladi Limestone; Dtv, Trout Valley Formation; Du, unnamed Devonian strata; Dw, Wapske Formation; Sb, Burtts Corners beds; Scc, Cross Creek beds; Scl, Canterbury Limestone; Scq, Clough Quartzite; Sd, Dennys Formation; Se, Edmunds Formation; Sf, Fitch Formation; Sfr, 
ingly calcareous to the southwest in western Maine and New Hampshire. Paleocurrents clearly show a southwesterly direction of flow, along the basin axis (Bradley and Hanson, 1989). The gradationally overlying Carrabassett Formation consists of another kilometer or so of chaotic, pelitic olistostrome with subordinate, coherent sand and silt turbidites. Paleocurrents show overall northerly flow (Hanson and Bradley, 1993). Hanson and Bradley (1989) interpreted the Madrid and Carrabassett Formations as having been deposited along a forelandbasin axis and on a north-facing slope, respectively; however, neither the Madrid nor the Carrabassett Formation has yielded useful fossils. The Madrid Formation is early Ludlow or younger. The Carrabassett Formation has yielded nondiagnostic brachiopods and plant fragments consistent with an Early Devonian age (Espenshade and Boudette, 1967, p. F12-F13), and is cut by the earliest Emsian Sebec Lake pluton.

The depositional history of the central part of the Central Maine Basin is not as well understood as that of the northwest flank because we lack detailed sedimentologic studies and deformation and metamorphism in the basin are more intense. A few observations bear on the timing of Acadian deformation. Most of the area is underlain by the Sangerville Formation, an isoclinally folded succession of siliciclastic and calcareous turbidites many kilometers thick. The Sangerville

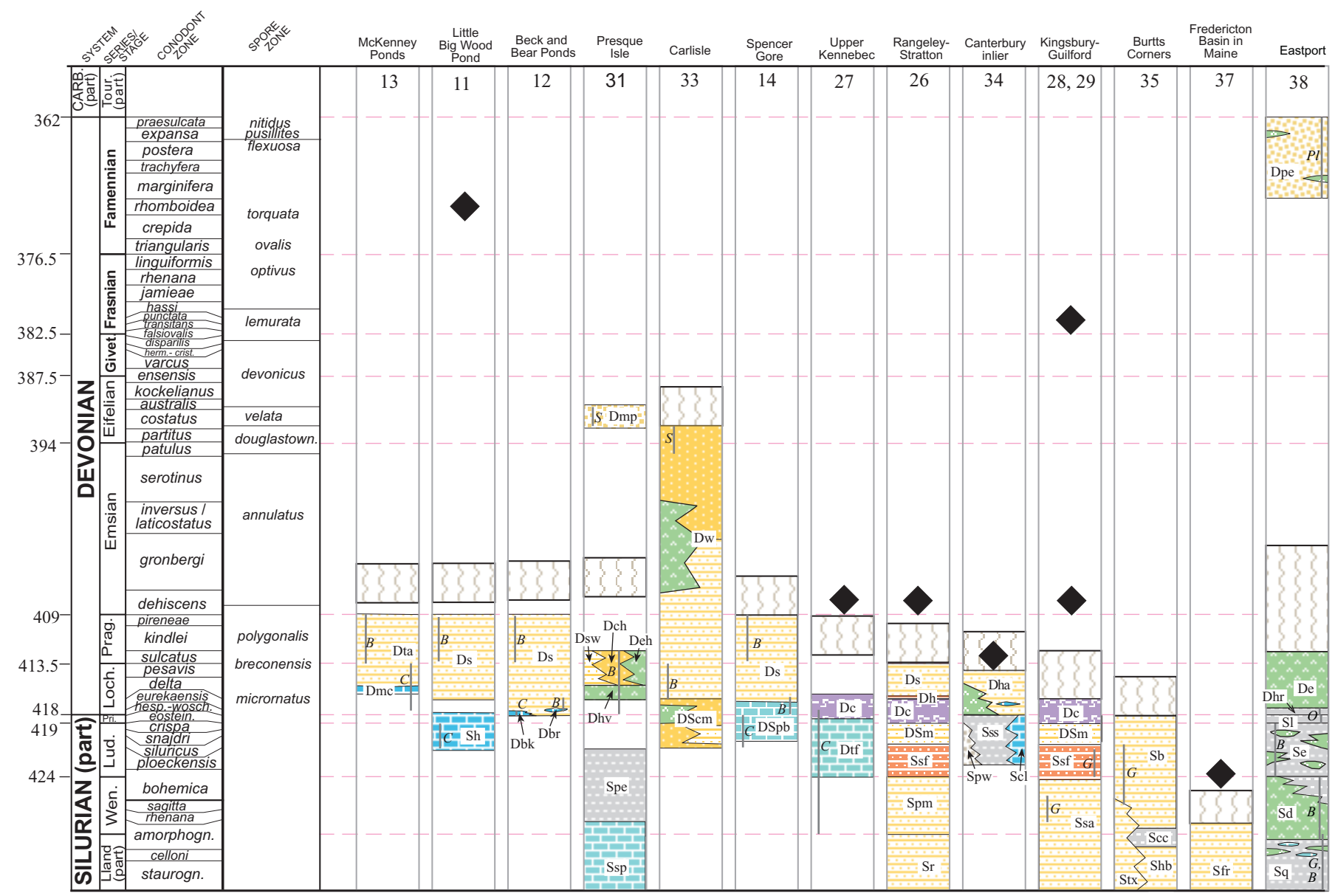

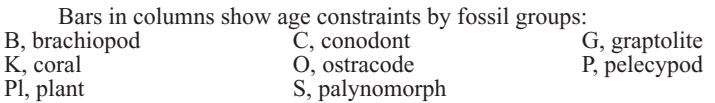

Flume Ridge Formation; Sgls, Grand Lake Seboeis Formation; Sh, Hardwood Mountain Formation; Shb, Hayes Brook beds; SI, Leighton Formation; SOc, Cabano Formation; SOm, Matapedia Group; Spe, Perham Formation; Spm, Perry Mountain Formation; Spw, Pocowogamis Conglomerate; Sq, Quoddy Formation; Sr, Rangeley Formation; Srp, Ripogenus Formation; Ssa, Sangerville Formation; Ssf, Smalls Falls Formation; Ssp, Spragueville Formation; Sss, Scott Siding Slate; Stx, Taxis River beds; Su, unnamed Silurian strata. References (numbers at top of columns): 1, Boucot and others (1986); 3, 4, Hueber and others (1990); 5, Uyeno and Lespérance (1997); 7, Uyeno and Lespérance (1997): 9, 10, Boucot and Arndt (1960); 11, Albee and Boudette (1972) and this report; 12, Boucot and others (1959) and this report; 13, Boucot and Heath (1969) and this report; 14, Boucot and Heath (1969) and this report; 15, Boucot and Heath (1969); 16, 17, Griscom (1976), Rankin (1968), and this report; 18, Hall (1970); 20, Hibbard (1993); 21, 22, Boone (1970) and this report; 23, this report; 24, St. Peter and Boucot (1981); 26, Moench and Pankiwksyj (1988); 27, Marvinney (1984) and this report; 28, 29, Pankiwskyj and others (1976), Ludman (1978) and this report; 31, Boucot and others (1964) and this report; 33, St. Peter (1982); 34, Venugopal (1979); 35, Fyffe (1995); 37, West and others (1992); 38, Gates and Moench (1981). 


\begin{tabular}{|c|c|c|c|c|c|c|c|c|}
\hline $\begin{array}{r}\text { Rock } \\
\text { unit }\end{array}$ & $\underset{\text { number }}{\text { GSC }}$ & $\begin{array}{l}\text { Station, } \\
\text { collector }\end{array}$ & $\begin{array}{l}\text { Location } \\
\text { (fig. 1) }\end{array}$ & $\begin{array}{l}\text { Latitude, } \\
\text { Longitude }\end{array}$ & Location & Lithology and structure & Age & Reference \\
\hline $\begin{array}{l}\text { Fish River Lake } \\
\text { Formation. }\end{array}$ & $0-106531$ & $\begin{array}{l}\text { 95MDw116A, } \\
\text { Bradley }\end{array}$ & 22 & $\begin{array}{l}47^{\circ} 00^{\prime} 52^{\prime \prime} \\
68^{\circ} 35^{\prime} 18^{\prime \prime}\end{array}$ & $\begin{array}{l}\text { Eagle Lake } 7.5^{\prime} \text { quadrangle. } \\
\text { Nadeau Thoroughfare railroad } \\
\text { cuts, } 90-120 \mathrm{~m} \mathrm{NE} \text {. of bridge. }\end{array}$ & $\begin{array}{l}\text { Medium sandstone, friable, plant } \\
\text { bearing, } 65^{\circ} \text { bedding dip, no } \\
\text { cleavage }\end{array}$ & $\begin{array}{l}\text { Most likely, and no older than, mid-late Emsian to very } \\
\text { early Eifelian, douglastownense-eurypterota Zone. } \\
\text { Spores from succeeding velata-langii Zone, early } \\
\text { Eifelian, are absent. }\end{array}$ & $\begin{array}{c}\text { McGregor } \\
\text { (1996) }\end{array}$ \\
\hline Do. & $0-106532$ & $\begin{array}{l}\text { 95MDw117, } \\
\text { Bradley }\end{array}$ & 22 & $\begin{array}{l}47^{\circ} 01^{\prime} 04^{\prime \prime} \\
68^{\circ} 35^{\prime} 05^{\prime \prime}\end{array}$ & $\begin{array}{l}\text { Eagle Lake } 7.5^{\prime} \text { quadrangle. } \\
\text { Nadeau Thoroughfare railroad } \\
\text { cuts, } 490-520 \mathrm{~m} \text { NE. of bridge. }\end{array}$ & $\begin{array}{l}\text { Medium sandstone, micaceous, } \\
\text { friable, plant bearing, } 51^{\circ} \text { bedding } \\
\text { dip, no cleavage. }\end{array}$ & $\begin{array}{l}\text { Most likely, and no older than, mid late Emsian to very } \\
\text { early Eifelian, douglastownense-eurypterota Zone. } \\
\text { Acritarchs suggest marine waters. }\end{array}$ & Do. \\
\hline Do. & $0-106533$ & $\begin{array}{l}\text { 95MDw118, } \\
\text { Bradley }\end{array}$ & 22 & $\begin{array}{l}47^{\circ} 01^{\prime} 14^{\prime \prime} \\
68^{\circ} 34^{\prime} 53^{\prime \prime}\end{array}$ & $\begin{array}{l}\text { Eagle Lake } 7.5^{\prime} \text { quadrangle. } \\
\text { Nadeau Thoroughfare railroad } \\
\text { cuts, } 910-950 \mathrm{~m} \text { NE. of bridge. }\end{array}$ & $\begin{array}{l}\text { Medium sandstone, micaceous, } \\
\text { friable, plant bearing, } 50^{\circ} \text { bedding } \\
\text { dip, no cleavage. }\end{array}$ & $\begin{array}{l}\text { Probably assignable to, and no older than, mid late } \\
\text { Emsian to very early Eifelian, douglastownense- } \\
\text { eurypterota Zone. Acritarchs suggest marine waters. }\end{array}$ & Do. \\
\hline Do. & $0-106536$ & $\begin{array}{l}\text { 95MDw124, } \\
\text { Bradley }\end{array}$ & 22 & $\begin{array}{l}47^{\circ} 02^{\prime} 30^{\prime \prime} \\
68^{\circ} 33^{\prime} 48^{\prime \prime}\end{array}$ & $\begin{array}{l}\text { Eagle Lake } 7.5^{\prime} \text { quadrangle. Eagle } \\
\text { Lake, south shore, near Camps of } \\
\text { Acadia. }\end{array}$ & $\begin{array}{l}\text { Gray micaceous siltstone, } 65^{\circ} \\
\text { bedding dip, strong cleavage. }\end{array}$ & Spores present, Late Silurian or younger--- & Do. \\
\hline Do. & $0-106537$ & $\begin{array}{l}\text { 95MDw125, } \\
\text { Bradley }\end{array}$ & 22 & $\begin{array}{l}47^{\circ} 00^{\prime} 52^{\prime \prime} \\
68^{\circ} 35^{\prime} 44^{\prime \prime}\end{array}$ & $\begin{array}{l}\text { Eagle Lake } 7.5^{\prime} \text { quadrangle. } \\
\text { Nadeau Thoroughfare, under } \\
\text { Route } 11 \text { bridge. }\end{array}$ & $\begin{array}{l}\text { Gray siltstone, outcrop-scale folds } \\
\text { with axial-planar cleavage. }\end{array}$ & $\begin{array}{l}\text { Probably middle Pragian to earliest Emsian, } \\
\text { polygonalisemsiensis Zone. Could be next younger } \\
\text { zone, but less likely. }\end{array}$ & Do. \\
\hline Do. & 6274 & $\begin{array}{l}\text { MK-62-8, } \\
\text { Mencher }\end{array}$ & 22 & $\begin{array}{l}47^{\circ} 58^{\prime} 37^{\prime \prime} \\
68^{\circ} 38^{\prime} 09^{\prime \prime}\end{array}$ & $\begin{array}{l}\text { Island Pond } 7.5^{\prime} \text { quadrangle. St. } \\
\text { Froid Lake, W. shore; exact } \\
\text { location uncertain. }\end{array}$ & $\begin{array}{l}\text { Lithology unknown. Strongly } \\
\text { cleaved, gray, micaceous siltstone } \\
\text { observed near here in } 1995 .\end{array}$ & Early Eifelian, douglastownense-eurypterota Zone & $\begin{array}{l}\text { McGregor } \\
\text { (1968). }\end{array}$ \\
\hline Do. & 6309 & $\begin{array}{l}\text { MK-62-9, } \\
\text { Mencher }\end{array}$ & 22 & $\begin{array}{l}47^{\circ} 58^{\prime} 35^{\prime \prime} \\
68^{\circ} 38^{\prime} 09^{\prime \prime}\end{array}$ & $\begin{array}{l}\text { Island Pond } 7.5^{\prime} \text { quadrangle. St. } \\
\text { Froid Lake, W. shore, } 30 \mathrm{~m} \mathrm{~S} \text {. of } \\
\text { MK-62-8; exact location } \\
\text { uncertain. }\end{array}$ & $\begin{array}{l}\text { Lithology unknown. Strongly } \\
\text { cleaved, gray, micaceous siltstone } \\
\text { observed near here in } 1995 .\end{array}$ & $\begin{array}{l}\text { Late Emsian to early Eifelian, douglastownense- } \\
\text { eurypterota Zone. }\end{array}$ & $\begin{array}{c}\text { McGregor } \\
(1962)\end{array}$ \\
\hline Do. & 6612 & $\begin{array}{l}\text { CL-186, } \\
\text { Mencher }\end{array}$ & 40 & $\begin{array}{l}46^{\circ} 58^{\prime} 26^{\prime \prime} \\
68^{\circ} 34^{\prime} 32^{\prime \prime}\end{array}$ & $\begin{array}{l}\text { Winterville } 7.5^{\prime} \text { quadrangle. Route } \\
11,480 \mathrm{~m} \mathrm{~N} \text {. of Winterville. }\end{array}$ & Lithology unknown; float sample. & Early to middle Eifelian, velata-langii Zone & $\begin{array}{l}\text { McGregor } \\
(1963)\end{array}$ \\
\hline $\begin{array}{l}\text { Fish River Lake } \\
\text { Formation. }\end{array}$ & 7754 & $\begin{array}{l}\text { EM-82, } \\
\text { Mencher }\end{array}$ & 22 & $\begin{array}{l}47^{\circ} 02^{\prime} 58^{\prime \prime} \\
68^{\circ} 30^{\prime} 02^{\prime \prime}\end{array}$ & $\begin{array}{l}\text { Eagle Lake } 7.5^{\prime} \text { quadrangle. Eagle } \\
\text { Lake, N. shore, } 80 \mathrm{~m} \text { from east } \\
\text { boundary of quadrangle. }\end{array}$ & Unknown-- & Middle Pragian or early Emsian- & $\begin{array}{l}\text { McGregor } \\
(1968)\end{array}$ \\
\hline Do. & 7760 & $\begin{array}{l}\text { EM-980, } \\
\text { Mencher }\end{array}$ & 22 & $\begin{array}{l}47^{\circ} 57^{\prime} 24^{\prime \prime} \\
68^{\circ} 39^{\prime} 38^{\prime \prime}\end{array}$ & $\begin{array}{l}\text { Island Pond } 7.5^{\prime} \text { quadrangle. Red } \\
\text { River, } 640 \mathrm{~m} \text { S. of Labbe Brook. }\end{array}$ & Unknown-_- & Emsian- & Do. \\
\hline Mapleton Formation. & 6275 & $\begin{array}{l}\text { MK-62-10, } \\
\text { Mencher }\end{array}$ & 31 & $\begin{array}{l}46^{\circ} 46^{\prime} 51^{\prime \prime} \\
68^{\circ} 04^{\prime} 57^{\prime \prime}\end{array}$ & $\begin{array}{l}\text { Presque Isle } 15^{\prime} \text { quadrangle. Same } \\
\text { locality as Stop } 2 \text { of Roy and } \\
\text { White (1987). }\end{array}$ & Unknown-__________- & Early to middle Eifelian, velata-langii Zone & $\begin{array}{l}\text { McGregor } \\
(1992)\end{array}$ \\
\hline $\begin{array}{l}\text { Trout Valley } \\
\text { Formation. }\end{array}$ & $0-105776$ & $\begin{array}{l}\text { Unknown, } \\
\text { Mencher }\end{array}$ & 17 & Unknown & Unknown- & Unknown-- & $\begin{array}{l}\text { Late Emsian to early Eifelian, douglastownense- } \\
\text { eurypterota Zone. }\end{array}$ & Do. \\
\hline $\begin{array}{l}\text { Unit DSus of Osberg } \\
\text { and others (1985). }\end{array}$ & $0-106534$ & $\begin{array}{l}\text { 95MDw119, } \\
\text { Bradley }\end{array}$ & 23 & $\begin{array}{l}47^{\circ} 06^{\prime} 36^{\prime \prime}, \\
68^{\circ} 22^{\prime} 57^{\prime \prime}\end{array}$ & $\begin{array}{l}\text { Square Lake } 7.5^{\prime} \text { quadrangle. } 2.2 \\
\text { km N. of Burnt Landing on } \\
\text { Square Lake access road. }\end{array}$ & $\begin{array}{l}\text { Tan laminated siltstone, } 90^{\circ} \text { bedding } \\
\text { dip, weak cleavage. }\end{array}$ & Small spores suggest Late Silurian to Lochkovian-....... & $\begin{array}{l}\text { McGregor } \\
(1996)\end{array}$ \\
\hline Do. & $0-106535$ & $\begin{array}{l}\text { 95MDw120, } \\
\text { Bradley }\end{array}$ & 23 & $\begin{array}{l}47^{\circ} 07^{\prime} 26^{\prime \prime} \\
68^{\circ} 22^{\prime} 52^{\prime \prime}\end{array}$ & $\begin{array}{l}\text { Square Lake } 7.5^{\prime} \text { quadrangle. } 4.7 \\
\mathrm{~km} \mathrm{~N} \text { of Burnt Landing on } \\
\text { Square Lake access road. }\end{array}$ & $\begin{array}{l}\text { Dark siltstone, } 80^{\circ} \text { bedding dip, } \\
\text { weak cleavage. }\end{array}$ & $\begin{array}{l}\text { Undoubtedly Early Devonian, probably Lochkovian } \\
\text { or early Pragian. }\end{array}$ & Do. \\
\hline Do. & 7758 & $\begin{array}{l}\text { EM-856, } \\
\text { Mencher }\end{array}$ & 41 & $\begin{array}{l}\text { Approx } \\
46^{\circ} 57^{\prime} \\
68^{\circ} 27^{\prime}\end{array}$ & $\begin{array}{l}\text { Portage } 15^{\prime} \text { quadrangle. Lumber } \\
\text { road approx. } 5.4 \mathrm{~km} \text { and } 1.3 \\
\mathrm{~km} \mathrm{E} \text {. from } \mathrm{NW} \text {. corner of } \\
\text { quadrangle. }\end{array}$ & Unknown- & Probably Pragian-- & $\begin{array}{l}\text { McGregor } \\
\text { (1968). }\end{array}$ \\
\hline Do. & 7766 & $\begin{array}{l}\text { DR-1, } \\
\text { Mencher }\end{array}$ & 42 & $\begin{array}{l}46^{\circ} 37^{\prime} 24^{\prime \prime} \\
68^{\circ} 24^{\prime} 17^{\prime}\end{array}$ & $\begin{array}{l}\text { Ashland } 15^{\prime} \text { quadrangle. Sheridan } \\
\text { Road opposite Ashland } \\
\text { Cemetery in Ashland. }\end{array}$ & Unknown- & Probably Pragian or Emsian- & Do. \\
\hline
\end{tabular}


has yielded graptolites from 13 localities (Pankiwskyj and others, 1976); the most diagnostic collection (loc. 28, figs. 1, 4 ) is middle Wenlock. The overlying Smalls Falls Formation has also yielded graptolites from several localities; the most diagnostic collection (loc. 29, figs. 1, 4) is early Ludlow (Pankiwskyj and others, 1976). The overlying Madrid and Carrabassett Formations are the youngest units along the central part of the basin. The Carrabassett Formation is absent in the most southerly strike belt of Madrid Formation either because it has since been eroded away (Moench and Pankiwskyj, 1988) or because it was never deposited (Hanson and Bradley, 1989).

\section{Littleton Area, New Hampshire}

The classic Silurian-Devonian section near Littleton, New Hampshire (loc. 9, figs. 1, 4; Billings, 1937), constrains the timing of both Acadian foreland-basin sedimentation and deformation. The Lower Silurian Clough Quartzite unconformably overlies Ordovician rocks and is overlain by the Fitch Formation, which consists of calcareous metasiltstone, quartzite, granofels, and some limestone. Harris and others (1983, p. 731) reported an age of late Ludlow to mid-Pridoli for the Fitch Formation in the Littleton area, on the basis of a conodont fauna representing the Ozarkodina remscheidensis eosteinhornensis Zone; along strike in Massachusetts, strata assigned to the Fitch Formation are as young as the woschmidti to eurekaensis Zones of the Lochkovian (Elbert and others, 1988). The Clough and Fitch are relatively thin, shallow-marine deposits; the Fitch has been interpreted as a far-foreland deposit (Bradley, 1983). The overlying Littleton Formation is an approximately $1.6-\mathrm{km}-$ thick flysch succession (a minimum thickness, because the top is not preserved) deposited in a foreland-basin setting (Bradley, 1983). It has yielded Emsian brachiopods at two localities in the Littleton area (loc. 9, figs. 1,4) and at a third locality in Whitefield (loc. 10, figs. 1, 4), where the fossils occur only a few tens of meters above the base (Boucot and Arndt, 1960, p. 41-43). At Gale River (loc. 39, fig. 1) in the next strike belt to the east, a tuff from low in the Littleton Formation has yielded an Emsian U-Pb zircon age of $407 \pm 2$ Ma (R.D. Tucker and D. Rankin, unpub. data, 1998). Still farther east, at Beaver Brook (loc. 8, fig. 1), a brachiopod fauna suggests a slightly older age ("Oriskany, possibly Esopus"; Boucot and Rumble, 1980, p. 192-194) than elsewhere (locs. 9, 10, fig. 1). The Littleton thus appears to be diachronous across strike at the latitude of its type area.

\section{Southwestern Part of the Moose River Synclinorium, Maine}

In the southwestern part of the Moose River synclinorium, western interior Maine, local carbonate-bearing units overlie Ordovician and older rocks, providing good age constraints on the onset of Acadian foreland-basin sedimentation. At Beck Pond (loc. 12, figs. 1, 4), the post-Ordovician section begins with the Beck Pond Limestone (Boucot and others, 1959) and includes reef limestone intercalated with boulder conglomerate derived from the nearby Attean pluton of Ordo- vician age. A rich brachiopod fauna initially suggested an "upper Helderberg, New Scotland" age (Boucot and Heath, 1969); however, conodonts from the upper part of the Beck Pond (member 5 of Boucot and others, 1959) include Belodella sp., Decoriconus sp., Dvorakia sp., Icriodus sp. indet., and Ozarkodina remscheidensis eosteinhornensis, indicating, instead, a very latest Pridoli to early Lochkovian age (pl. 1, figs. 1-13; table 2). The Beck Pond Limestone is overlain by a considerable thickness, probably 2 to $3 \mathrm{~km}$, of Lower Devonian flysch assigned to the Seboomook Group (formerly Formation; Pollock, 1987). Boucot and others (1959) interpreted the contact between Member 5 of the Beck Pond and overlying slate of the Seboomook as an angular unconformity. Upon digging out this contact (at loc. 10 of Boucot and others, 1959), we observed, instead, that reefal limestone debris interfingers with black silty shale. The conodonts thus date the top of the Beck Pond Limestone, the base of the Seboomook flysch sequence, and the onset of Acadian foreland-basin sedimentation.

About $1 \mathrm{~km}$ to the north (loc. 12, figs. 1, 4), the Bear Pond Limestone (Boucot and others, 1959) is a carbonate lens or buildup not far above the base of the Seboomook Group. It is known from a single outcrop that exposes about $10 \mathrm{~m}$ of section. A rich brachiopod fauna again indicates an "upper Helderberg, New Scotland" age (Boucot and Heath, 1969) for the buildup and, by implication, for the immediately overlying and underlying siltstone and sandstone of the Seboomook Group. A large conodont sample yielded a meager conodont fauna of Belodella devonica, Ozarkodina remscheidensis eosteinhornensis, and fragments of Icriodus sp. indet. or Pedavis sp. indet., allowing a possible age range from late Ludlow to earliest Pragian (table 2). Nearby, Boucot and Heath (1969, p. 36) reported several brachiopod occurrences that suggest a Pragian age for all but the lowest beds of the Seboomook Group in the Moose River synclinorium. The Seboomook Group is the youngest pre-Acadian stratified unit in the area; it is not known to extend upward beyond the Pragian.

About $15 \mathrm{~km}$ to the northeast, the post-Ordovician section (loc. 13, figs. 1, 4) begins with the McKenney Ponds Limestone Member of the Tarratine Formation. Boucot and Heath (1969) reported a "Becraft-Oriskany" (late Lochkovian to Pragian) age based on brachiopods. This assignment can be somewhat refined on the basis of our conodont collections (table 2). The most diagnostic of three samples (USGS colln. 12516-SD, table 2), located 2 m stratigraphically below siltstone of the Tarratine Formation, yielded Belodella devonica, Icriodus sp. indet., Ozarkodina remscheidensis, and Pseudooneotodus beckmanni (pl. 1, figs. 19-26). A Lochkovian, but not earliest Lochkovian, age is indicated by the icriodids, which are of a post-I. woschmidtii morphotype. The limestone member is gradationally overlain by sandstone of the main body of the Tarratine Formation, which both interfingers with and overlies the Seboomook Group and likewise is interpreted as part of the forelandbasin fill. The Tarratine Formation has yielded Oriskany-age (Pragian) brachiopods from several nearby localities (Boucot and Heath, 1969). 


\section{Northeastern Part of the Moose River Synclinorium, Maine}

The northeastern part of the Moose River synclinorium in Maine provides crucial stratigraphic constraints on the age of the youngest foreland-basin deposits. In the area of northern Moosehead and Brassua Lakes (loc. 15, figs. 1, 4), Ordovician and older basement rocks are overlain by poorly dated Silurian calcareous rocks and Devonian(?) red shale (Boucot and Heath, 1969). The Acadian foreland-basin succession begins with the Tarratine Formation, which is about $2 \mathrm{~km}$ thick in this area (Boucot and Heath, 1969, pl. 19). The Tarratine Formation has yielded abundant Oriskany-age — that is, Pragian—brachiopods (Boucot and Heath, 1969, p. 27). The Kineo Rhyolite, sandwiched between the Tarratine Formation, below, and the Tomhegan Formation, above, represents a pulse of silicic magmatism in the foreland basin. The Kineo Rhyolite has yielded only discordant zircon ages, marked by inheritance. It is comparable in stratigraphic position, composition, and eruptive environment to the 407- to 406-Ma Traveler Rhyolite (Rankin and Tucker, 1995) $65 \mathrm{~km}$ to the northeast (loc. 17, figs. 1, 4); the Kineo and Traveler are presumably coeval. The Kineo ranges in thickness from $0 \mathrm{~m}$ at its north limit to about 1,200 $\mathrm{m}$ just a few kilometers to the south. It is overlain by the main body of the Tomhegan Formation, consisting of crossbedded, probably deltaic sandstone (L. Hanson and D. Bradley, unpub. data). Boucot and Heath (1969, p. 17) estimated the thickness at about $1,800 \mathrm{~m}$-a minimum, because the top is not preserved. Brachiopods in the Tomhegan indicate a "Schoharie" (late Emsian; Boucot and Heath, 1969, p. 20) age. The Tomhegan is the youngest pre-Acadian unit in this part of Maine, indicating that the Acadian deformation here was no earlier than the late Emsian.

\section{Traveler-Chesuncook Area, Maine}

Along the west side of the Katahdin batholith (loc. 16, figs. 1, 4), Silurian sedimentation began with deposition of shallow marine conglomerate, limestone, and siltstone assigned by Griscom (1976) to the Ripogenus Formation. Various localities within the Ripogenus Formation have yielded brachiopods of Llandovery (C3-C5), late Wenlock, and possible Ludlow age (Boucot and Heath, 1969, p. 53). A conodont sample (95MDW130) from Ripogenus Dam (loc. 16, figs. 1, 4; table 2) yielded Decoriconus sp. indet., Dvorakia sp. indet., Oulodus sp. indet., Ozarkodina excavata, and Panderodus sp. indet. (pl. 2, figs. 1-5); this collection permits an age range of late Ludlow to early Lochkovian. The Ripoge-

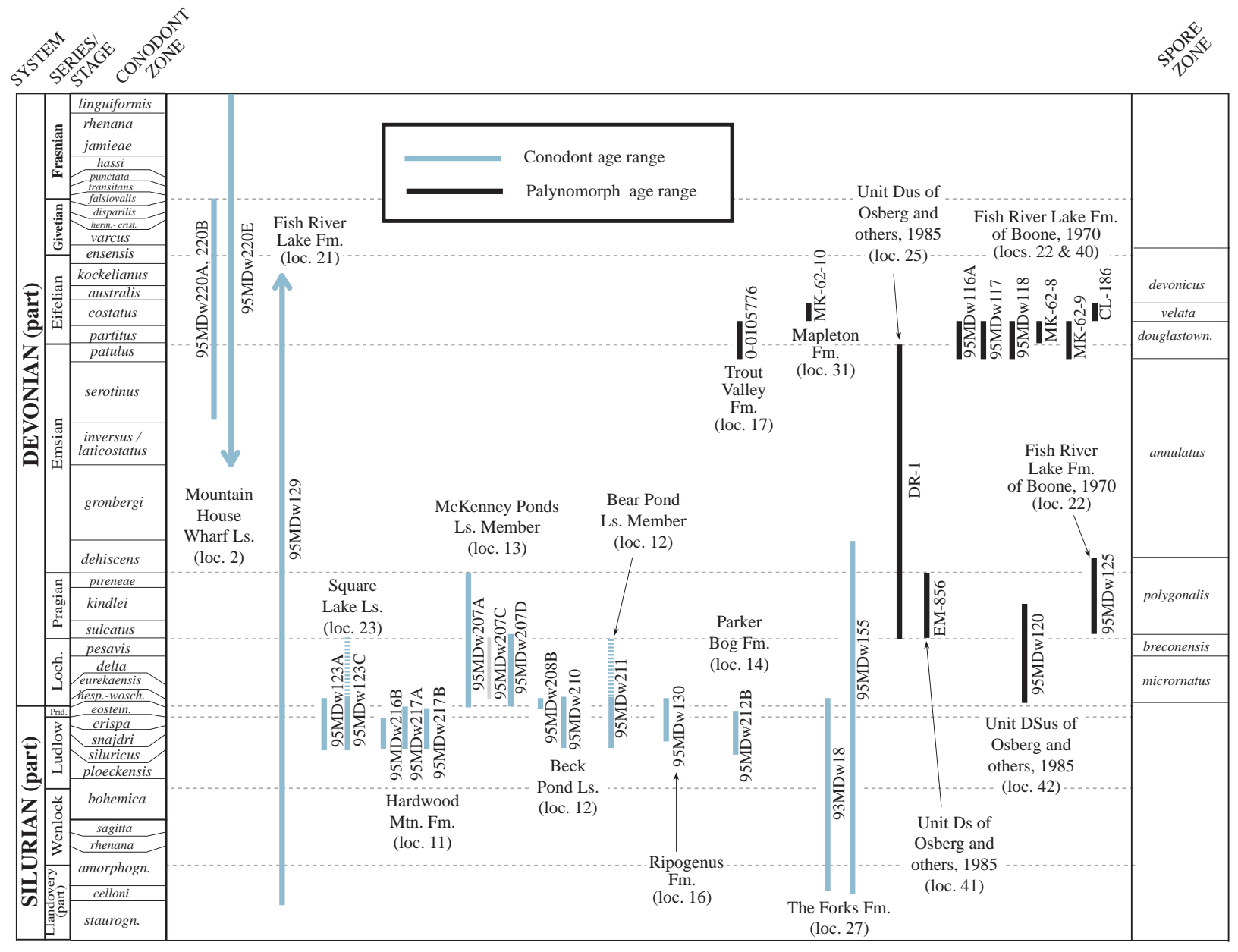

Figure 5.-Correlation chart showing conodont age ranges for samples dated in this report, and palynomorph age ranges for samples dated in this report and for samples from northern Maine that were previously discussed in internal paleontologic reports of the Geological Survey of Canada (McGregor, 1962, 1963, 1968, 1992). 
Table 2. Conodont collections from Silurian and Devonian rocks, Maine and Québec.

[See plates 1 and 2 for illustrations of key conodonts; see figure 5 for the Silurian and Devonian conodont zonation and a graphical display of the age range of each sample. CAI, conodont-alteration index; USGS, U.S. Geological Survey]

\begin{tabular}{|c|c|c|c|c|c|c|c|c|}
\hline $\begin{array}{l}\text { Loc. no. } \\
\text { in fig. } 1\end{array}$ & $\begin{array}{l}\text { Field no. } \\
\text { (USGS } \\
\text { colln. no.) }\end{array}$ & $\begin{array}{l}\text { County } \\
\text { quadrangle } \\
\text { lat./long. }\end{array}$ & $\begin{array}{l}\text { Formation, lithology, and } \\
\text { locality description }\end{array}$ & Conodonts & Age & Biofacies & CAI & Remarks \\
\hline 23 & $\begin{array}{l}\text { 95MDw123A } \\
\text { (12511-SD) }\end{array}$ & $\begin{array}{l}\text { Aroostook } \\
\text { Square Lake } \\
\text { West } \\
47^{\circ} 03^{\prime} 52^{\prime \prime \prime} \\
68^{\circ} 22^{\prime} 57^{\prime \prime}\end{array}$ & $\begin{array}{l}\text { Square Lake Limestone- } \\
\text { prominent lakeshore } \\
\text { outcrop of biostromal } \\
\text { limestone containing } \\
\text { abundant brachiopods, } \\
\text { corals, crinoids, and } \\
\text { bryozoans. }\end{array}$ & $\begin{array}{l}145 \text { Belodella spp. including B. cf. B. resima (Philip) (pl. 2, } \\
\text { figs. 27-32) } \\
21 \text { Decoriconus fragilis (Branson and Mehl) (pl. 1, figs. } \\
22-26 \text { ) } \\
4 \text { Dvorakia sp. (pl. 2, figs. 36, 37) } \\
\text { Ozarkodina excavata (Branson and Mehl)? } \\
\text { 1 Sa and } 1 \text { Sb element fragments } \\
\text { Ozarkodina remscheidensis remscheidensis (Ziegler) } \\
10 \text { Pa, } 5 \text { Pb, } 2 \text { M, and } 2 \text { Sc elements (chiefly fragments) } \\
9 \text { Pseudooneotodus beckmanni (Bischoff and Sannemann) } \\
\text { (pl. 2, fig. 38) } \\
42 \text { indet. bar, blade, platform and coniform fragments }\end{array}$ & $\begin{array}{l}\text { late Ludlow-early } \\
\text { Lochkovian ( } \text { siluricus } \\
\text { Zone to at least } \\
\text { woschmidti Zone). }\end{array}$ & $\begin{array}{l}\text { Belodellid biofacies: } \\
\text { this collection, with } \\
\text { an abundance of } \\
\text { coniform elements, } \\
\text { is typical of reefoid } \\
\text { deposits in the } \\
\text { Silurian and earliest } \\
\text { Devonian. }\end{array}$ & 1.5 & $\begin{array}{l}8.7 \mathrm{~kg} \text { processed; } 4.02 \mathrm{~kg} \\
+20 \text { mesh and } 220 \mathrm{~g} \\
20-200 \text { mesh insoluble } \\
\text { residue. }\end{array}$ \\
\hline 23 & $\begin{array}{l}\text { 95MDw123C } \\
\text { (12512-SD) }\end{array}$ & $\begin{array}{l}\text { Aroostook } \\
\text { Square Lake } \\
\text { West } \\
47^{\circ} 03^{\prime} 52^{\prime \prime \prime} \\
68^{\circ} 22^{\prime} 57^{\prime \prime}\end{array}$ & $\begin{array}{l}\text { Square Lake Limestone- } \\
\text { grainstone, from low } \\
\text { outcrops at water line } \\
\text { about } 50 \mathrm{~m} \text { west and } \\
\text { stratigraphically above } \\
\text { 95MDw123A. }\end{array}$ & $\begin{array}{l}1 \mathrm{~Pb} \text { Oulodus } \mathrm{sp} . \text { indet. } \\
\text { Ozarkodina remscheidensis (Ziegler) } \\
8 \mathrm{~Pa}(\text { o. r. remscheidensis }), 1 \mathrm{~Pa}(\text { O. r. eosteinhornensis }), 1 \\
\mathrm{~Pb}, 1 \mathrm{Sa} \text { and } 1 \mathrm{Sc} \text { elements (chiefly fragments) } \\
15 \text { indet. bar, blade, and platform fragments }\end{array}$ & $\begin{array}{l}\text { late Ludlow through } \\
\text { earliest Pragian } \\
\text { (siluricus Zone into } \\
\text { lowermost sulcatus } \\
\text { Zone }\end{array}$ & $\begin{array}{l}\text { Indeterminate- } \\
\text { too few conodonts. } \\
\text { Conodonts indicate } \\
\text { high-energy, normal- } \\
\text { marine depositional } \\
\text { setting. }\end{array}$ & 1.5 & $\begin{array}{l}3.8 \mathrm{~kg} \text { processed; } 400 \mathrm{~g} \\
+20 \text { mesh and } 62 \mathrm{~g} 20-200 \\
\text { mesh insoluble residue. }\end{array}$ \\
\hline 21 & 95MDw129 & $\begin{array}{l}\text { Aroostook } \\
\text { Fish River Lake } \\
46^{\circ} 50^{\prime} 52^{\prime \prime \prime} \\
68^{\circ} 46^{\prime} 02^{\prime \prime}\end{array}$ & $\begin{array}{l}\text { Fish River Lake Forma- } \\
\text { tion-silty limestone } \\
\text { containing crinoid and } \\
\text { stromatoporoid debris. }\end{array}$ & $\begin{array}{l}2 \text { Sc element fragments of post-Ordovician Paleozoic morpho- } \\
\text { type } \\
\text { several phosphatic bryozoan pearls }\end{array}$ & $\begin{array}{l}\text { Middle Ordovician- } \\
\text { Mississippian on the } \\
\text { basis of the bryozoan } \\
\text { pearls. }\end{array}$ & $\begin{array}{l}\text { Indeterminate- } \\
\text { too few conodonts. }\end{array}$ & 3 & $\begin{array}{l}5.2 \mathrm{~kg} \text { processed; } 3.3 \mathrm{~kg} \\
+20 \text { mesh and } 466 \mathrm{~g} \\
20-200 \text { mesh insoluble } \\
\text { residue. }\end{array}$ \\
\hline 16 & $\begin{array}{l}\text { 95MDw130 } \\
\text { (12513-SD) }\end{array}$ & $\begin{array}{l}\text { Piscataquis } \\
\text { Harrington Lake } \\
45^{\circ} 52^{\prime} 55^{\prime \prime} / \\
69^{\circ} 10^{\prime} 38^{\prime \prime}\end{array}$ & $\begin{array}{l}\text { Ripogenus Formation- } \\
\text { limestone. Sample is from } \\
2 \text {-m-thick limestone with } \\
\text { shaly partings separated } \\
\text { from an overlying } 5-\mathrm{m}- \\
\text { thick conglomerate by } 1 \mathrm{~m} \\
\text { of calcareous sandstone. } \\
\text { At } 90^{\circ} \text { bend in Frost Pond } \\
\text { Rd. }\end{array}$ & $\begin{array}{l}2 \text { Decoriconus sp. indet. } \\
6 \text { Dvorakia sp. indet. (pl. 2, figs. 1, 2) } \\
1 \mathrm{~Pb} \text { element Oulodus sp. indet. } \\
\text { Ozarkodina excavata (Branson and Mehl) } \\
1 \mathrm{~Pa}, 1 \mathrm{M}, 1 \mathrm{Sa}, 6 \mathrm{Sb} \text {, and } 4 \mathrm{Sc} \text { elements (pl. 2, figs. 3, 4) } \\
59 \text { Panderodus sp. indet. } \\
1 \mathrm{Sb} \text { element of a digyrate apparatus (pl. 2, fig. 5) } \\
34 \text { indet. bar, blade, and platform fragments }\end{array}$ & $\begin{array}{l}\text { late Ludlow-early } \\
\text { Lochkovian (siluricus } \\
\text { Zone-earliest Early } \\
\text { Devonian) }\end{array}$ & $\begin{array}{l}\text { Panderodid } \\
\text { biofacies: normal- } \\
\text { marine, probably } \\
\text { relatively shallow- } \\
\text { water depositional } \\
\text { environment. }\end{array}$ & $\begin{array}{l}\text { chiefly } \\
5.5 ; \\
\text { minor } 5 \\
\text { and } 6\end{array}$ & $\begin{array}{l}11.5 \mathrm{~kg} \text { processed; } 4.7 \mathrm{~kg} \\
+20 \text { mesh and } 195 \mathrm{~g} \\
20-200 \text { mesh insoluble } \\
\text { residue. }\end{array}$ \\
\hline 27 & $\begin{array}{l}\text { 95MDw155 } \\
\text { (12514-SD) }\end{array}$ & $\begin{array}{l}\text { Somerset } \\
\text { The Forks } \\
45^{\circ} 19^{\prime} 58^{\prime \prime} / \\
69^{\circ} 58^{\prime} 05^{\prime \prime}\end{array}$ & $\begin{array}{l}\text { The Forks Formation-- } \\
\text { calcareous sandstone to } \\
\text { sandy limestone. Roadcut } \\
\text { on E side of US 201, 0.3 } \\
\text { mi S of Kennebec River } \\
\text { bridge; sample from } \\
\text { prominent 20-cm-thick, } \\
\text { white calcareous } \\
\text { sandstone. Stratigraph- } \\
\text { ically higher than } \\
\text { 95MDw180. }\end{array}$ & $\begin{array}{l}\text { Ozarkodina excavata (Branson and Mehl) } \\
5 \mathrm{~Pa}, 1 \mathrm{~Pb}, 1 \mathrm{M} \text {, and } 1 \mathrm{Sc} \text { elements } \\
46 \text { indet. bar, blade, and platform fragments }\end{array}$ & $\begin{array}{l}\text { late Llandovery-early } \\
\text { Emsian. }\end{array}$ & $\begin{array}{l}\text { Indeterminate- } \\
\text { too few conodonts. } \\
\text { Normal-marine, } \\
\text { relatively high- } \\
\text { energy depositional } \\
\text { setting. }\end{array}$ & 6.5 & $\begin{array}{l}12.1 \mathrm{~kg} \text { processed; } 4.9 \mathrm{~kg} \\
+20 \text { mesh and } 1.2 \mathrm{~kg} \\
20-200 \text { mesh insoluble } \\
\text { residue. }\end{array}$ \\
\hline 27 & $\begin{array}{l}\text { 93MDw18 } \\
\text { (12334-SD) }\end{array}$ & $\begin{array}{l}\text { Somerset } \\
\text { The Forks } \\
45^{\circ} 18.33^{\prime \prime} \\
69^{\circ} 59.2^{\prime}\end{array}$ & $\begin{array}{l}\text { The Forks Formation from } \\
\text { roadcut on E side of US } \\
201,4.9 \text { mi N of Caratunk. } \\
\text { Recessive calcareous } \\
\text { horizon } 75 \mathrm{ft} . \mathrm{N} \text { of } \\
\text { telephone pole no. } \\
234,445,131 .\end{array}$ & $\begin{array}{l}3 \text { Decoriconus sp. indet. } \\
\text { Ozarkodina excavata (Branson and Mehl) } \\
5 \mathrm{~Pa}, 2 \mathrm{M} \text {, and } 1 \mathrm{Sb} \text { elements } \\
2 \text { Panderodus sp. indet. } \\
33 \text { indet. bar, blade, and platform fragments } \\
\text { Phosphatized steinkerns: } \\
1 \text { gastropod and } 1 \text { pelmatozoan ossicle }\end{array}$ & $\begin{array}{l}\text { late Llandovery-early } \\
\text { Lochkovian. }\end{array}$ & $\begin{array}{l}\text { Indeterminate- } \\
\text { too few conodonts. } \\
\text { Preservation and } \\
\text { composition of fauna } \\
\text { suggest a high- } \\
\text { energy, normal- } \\
\text { marine setting. }\end{array}$ & $5.5-6.5$ & $\begin{array}{l}9.9 \mathrm{~kg} \text { processed; } 4.02 \mathrm{~kg} \\
+20 \text { mesh and } 737 \mathrm{~g} \\
20-200 \text { mesh insoluble } \\
\text { residue. }\end{array}$ \\
\hline
\end{tabular}


Table 2. Conodont collections from Silurian and Devonian rocks, Maine and Québec-Continued.

\begin{tabular}{|c|c|c|c|c|c|c|c|c|}
\hline $\begin{array}{l}\text { Loc. no. } \\
\text { in fig. } 1\end{array}$ & $\begin{array}{l}\text { Field no. } \\
\text { (USGS } \\
\text { colln. no.) }\end{array}$ & $\begin{array}{l}\text { County } \\
\text { quadrangle } \\
\text { lat./long. }\end{array}$ & $\begin{array}{l}\text { Formation, lithology, and } \\
\text { locality description }\end{array}$ & Conodonts & Age & Biofacies & CAI & Remarks \\
\hline 13 & $\begin{array}{l}\text { 95MDw207A } \\
\text { (12515-SD) }\end{array}$ & $\begin{array}{l}\text { Somerset } \\
\text { Enchanted Pond } \\
45^{2} 27^{\prime} 155^{\prime \prime} / \\
70^{\circ} 12^{2} 233^{\prime \prime}\end{array}$ & \begin{tabular}{l|} 
McKenney Ponds \\
Limestone Member of \\
Tarratine Formation. \\
Sample from middle of 3 \\
caves below outlet of pond \\
and $~ 5 \mathrm{~m}$ below lowest \\
exposure of Tarratine \\
Formation siltstone.
\end{tabular} & $\begin{array}{l}14 \text { Belodella devonica (Stauffer) } \\
8 \text { Dvorakia sp. indet. } \\
34 \text { I element fragments of Icriodus sp. indet. of Lochkovian- } \\
\text { Pragian morphotype (pl. 2, figs. } 16-18 \text { ) } \\
3 \text { Pseudooneotodus beckmanni (Bischoff and Sannemann) }\end{array}$ & Lochkovian-Pragian. & $\begin{array}{l}\text { Post mortem } \\
\text { transport within or } \\
\text { from the icriodid } \\
\text { biofacies; relatively } \\
\text { high-energy normal- } \\
\text { marine depositional } \\
\text { setting. }\end{array}$ & 5 & $\begin{array}{l}11.4 \mathrm{~kg} \text { processed; } 200 \mathrm{~g} \\
+200 \text { mesh and } 96 \mathrm{~g} 20-200 \\
\text { mesh insoluble residue. } \\
\text { Heavy-mineral } \\
\text { concentratec cheffly } \\
\text { phosphatic brachiopod } \\
\text { fragents and euhedral } \\
\text { pink zircons. }\end{array}$ \\
\hline 13 & $\begin{array}{l}\text { 95MDw207C } \\
\text { (12516-SD) }\end{array}$ & $\begin{array}{l}\text { Somerset } \\
\text { Enchanted Pond } \\
45^{\circ} 7^{\prime} 155^{\prime \prime \prime} \\
70^{\circ} 12^{2} 23^{\prime \prime}\end{array}$ & \begin{tabular}{l|} 
McKenney Ponds \\
Limestone Member of \\
Tarratine Formation. \\
Sample is from the \\
southwesterly of the 3 \\
caves and from an \\
irregularly bedded \\
bioclastic limestone, $3.5 \mathrm{~m}$ \\
stratigraphically above \\
95MDw207D.
\end{tabular} & $\begin{array}{l}37 \text { Belodella devonica (Stauffer) } \\
67 \text { I (all fragments) and } 5 \text { coniform elements of Icriodus sp. } \\
\text { indet of Lochkovian, but not early Lochkovian morphotype } \\
\text { (not I I woschmidti) (pl. 1, figs. 19-24) } \\
2 \text { Pa elements Ozarkodina remscheidensis (Ziegler) (pl. 1, } \\
\text { figs. 25, 26) } \\
2 \text { Pseudooneotodus beckmanni (Bischoff and Sannemann) }\end{array}$ & $\begin{array}{l}\text { Lochkovian, but not } \\
\text { early Lochkovian. }\end{array}$ & & 5 & $\begin{array}{l}19.5 \mathrm{~kg} \text { processed; } 20 \mathrm{~g} \\
+20 \text { mesh and } 77 \mathrm{~g} 20-200 \\
\text { mesh insoluble residue. } \\
\text { Heavy-mineral concentrate } \\
\text { includes phosphatic } \\
\text { brachiopod fragments. }\end{array}$ \\
\hline 13 & $\begin{array}{l}\text { 95MDw207D } \\
\text { (12517-SD) }\end{array}$ & $\begin{array}{l}\text { Somerset } \\
\text { Enchanted Pond } \\
45^{\circ} 27^{\prime} 15 " \prime \\
70^{\circ} 12^{2} 23^{\prime \prime}\end{array}$ & $\begin{array}{l}\text { McKenney Ponds } \\
\text { Limestone Member of } \\
\text { Tarratine Formation. } \\
\text { Sample is from the } \\
\text { southwesterly of the } 3 \\
\text { caves and from an } \\
\text { irregularly bedded } \\
\text { bioclastic cilimestone, about } \\
3.5 \mathrm{~m} \text { below 95MDW207C, } \\
\text { and from approximately } \\
\text { the same level as } \\
95 \mathrm{MDD} 207 \mathrm{~A} \text {. }\end{array}$ & $\begin{array}{l}14 \text { Belodella devonica (Stauffer) coniform elements } \\
1 \text { small Pelement fragment } \text { (criodus sp. indet. } \\
\text { Ozarkodina remschiedensis (Ziegler) } \\
23 \text { pa and } 1 \text { Pb elements } \\
1 \text { Pseudooneotodus beckmanni (Bischoff and Sannemann) } \\
32 \text { indet. bar, blade, and platform fragments }\end{array}$ & Lochkovian. & $\begin{array}{l}\text { Ozarkodinid } \\
\text { biofaciess normal- } \\
\text { marine, shelf } \\
\text { depositional } \\
\text { environment }\end{array}$ & 5 & $\begin{array}{l}10.7 \mathrm{~kg} \text { processed; } 180 \mathrm{~g} \\
+20 \text { mesh and } 29 \mathrm{~g} 20-200 \\
\text { mesh insoluble residee. } \\
\text { Heavy-mineral concentrate } \\
\text { includes phosphatic } \\
\text { brachiopod fragments. }\end{array}$ \\
\hline 12 & $\begin{array}{l}\text { 95MDw208B } \\
\text { (12518-SD) }\end{array}$ & $\begin{array}{l}\text { Somerset } \\
\text { King and } \\
\text { Bartlett Lake } \\
45^{\circ} 22^{\prime} 05^{\prime \prime \prime} \\
70^{\circ} 20^{\prime} 18^{\prime \prime}\end{array}$ & $\begin{array}{l}\text { Beck Pond Limestone. } \\
\text { Carbonate matrix } \\
\text { conglomerate with clasts } \\
\text { of granite and reef debris. } \\
\text { This is outcrop 10 of } \\
\text { Boucot and others (1959). } \\
\text { This sample is from the } \\
\text { youngest member (Bouct } \\
\text { and others' Member 5) of } \\
\text { the Beck Pond. }\end{array}$ & 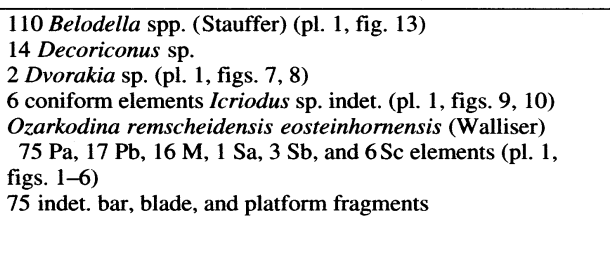 & $\begin{array}{l}\text { Very latest Pridoli- } \\
\text { early Lochkovian } \\
\text { (based on overlap of } \\
\text { icriodids and } \\
\text { Decoriconus). }\end{array}$ & $\begin{array}{l}\text { Ozarkodinid } \\
\text { biofacies: normal- } \\
\text { marine, shallow- } \\
\text { water shelf } \\
\text { depositional } \\
\text { environment. }\end{array}$ & $4.5-5$ & $\begin{array}{l}41.2 \mathrm{~kg} \text { processed; } 7.6 \mathrm{~kg} \\
+20 \text { mesh and } 1.7 \mathrm{~kg} \\
20-200 \text { mesh insoluble } \\
\text { residue. Heavy-mineral } \\
\text { concentrate includes } \\
\text { phosphatic zooecial linings } \\
\text { and pearls of bryozanans, } \\
\text { detrital muscovite, biotite, } \\
\text { chlorite, and euhedral } \\
\text { zircon. }\end{array}$ \\
\hline 12 & $\begin{array}{l}\text { 95MDw210 } \\
\text { (12519-SD) }\end{array}$ & $\begin{array}{l}\text { Somerset } \\
\text { King and } \\
\text { Bartlett Lake } \\
45^{2} 22^{2} 1111 ! \\
70^{2} 20^{4} 1^{\prime \prime}\end{array}$ & $\begin{array}{l}\text { Beck Pond Limestone. } \\
\text { Sandy limestone } \\
\text { containing abundant } \\
\text { bryozoans and corals. This } \\
\text { is the same locale as } \\
\text { outcrop } 32 \text { of Boucot and } \\
\text { others } 12999 \text { ) and is from } \\
\text { the oldest member (Boucot } \\
\text { and others' 'Member 1) of } \\
\text { the Beck Pond. }\end{array}$ & $\begin{array}{l}5 \mathrm{~Pa} \text { elements } \text { Ozarkodina remscheidensis eosteinhornensis } \\
\text { (Walliser) }\end{array}$ & $\begin{array}{l}\text { late Ludlow-early } \\
\text { Lochkovian (siluricus } \\
\text { Zone to at least } \\
\text { woschmidti Zone). }\end{array}$ & $\begin{array}{l}\text { Indeterminate- } \\
\text { too few conodonts. }\end{array}$ & $4.5-5$ & $\begin{array}{l}8.3 \mathrm{~kg} \text { processed; } 340 \mathrm{~g} \\
+20 \text { mesh and } 208 \mathrm{~g} \\
20-200 \text { mesh insoluble } \\
\text { residue. }\end{array}$ \\
\hline
\end{tabular}


Table 2. Conodont collections from Silurian and Devonian rocks, Maine and Québec_-Continued.

\begin{tabular}{|c|c|c|c|c|c|c|c|c|}
\hline $\begin{array}{l}\text { Loc. no. } \\
\text { in fig. } 1\end{array}$ & $\begin{array}{l}\text { Field no. } \\
\text { (USGS } \\
\text { colln. no.) }\end{array}$ & $\begin{array}{l}\text { County } \\
\text { quadrangle } \\
\text { lat./long. }\end{array}$ & $\begin{array}{l}\text { Formation, lithology, and } \\
\text { locality description }\end{array}$ & Conodonts & Age & Biofacies & CAI & Remarks \\
\hline 12 & $\begin{array}{l}\text { 95MDw211 } \\
\text { (12520-SD) }\end{array}$ & $\begin{array}{l}\text { Somerset } \\
\text { Spencer Lake } \\
45^{\circ} 22^{4} 40^{\prime \prime} \\
70^{\circ} 20^{\prime} 34^{\prime \prime}\end{array}$ & $\begin{array}{l}\text { Bear Pond Limestone } \\
\text { Member of Seboomook } \\
\text { Group (formerly } \\
\text { Seboomook Formation). } \\
\text { This is outcrop 42 of } \\
\text { Boucot and others (1959). }\end{array}$ & $\begin{array}{l}1 \text { Belodella devonica (Stauffer) } \\
1 \text { Pa element Ozarkodina remscheidensis eosteinhornensis } \\
\text { (Walliser) } \\
3 \text { P element fragments Icriodus sp. indet. or Pedavis sp. indet. }\end{array}$ & \begin{tabular}{l|} 
late Ludlow-earliest \\
Pragian (no older than \\
siluricus Zone)
\end{tabular} & $\begin{array}{l}\text { Indeterminate- } \\
\text { too few conodonts. }\end{array}$ & 5 & $\begin{array}{l}11.1 \mathrm{~kg} \text { processed; } 1.43 \mathrm{~kg} \\
+20 \text { mesh and 2.2 } \mathrm{kg} \\
20-200 \text { mesh insoluble } \\
\text { residue. Heavy-mineral } \\
\text { concentrate includes } \\
\text { phosphatic zooecial linings } \\
\text { of bryozoans and ling } \\
\text { phosphatic tubes, and rare } \\
\text { euhedral zircon. }\end{array}$ \\
\hline 14 & $\begin{array}{l}\text { 95MDw212B } \\
\text { (12521-SD) }\end{array}$ & $\begin{array}{l}\text { Somerset } \\
\text { King and } \\
\text { Bartlett Lake } \\
\sim 45^{\circ} 18.7^{\prime} / \\
\sim 70^{\circ} 19.6^{\prime}\end{array}$ & $\begin{array}{l}\text { Parker Bog Formation- } \\
\text { limestone interbedded with } \\
\text { siliceous siltstone or tuff. } \\
\text { From south bank of } \\
\text { Spencer Streanm detailed } \\
\text { outcrop map in Buocot and } \\
\text { others (1958). From the } \\
\text { thickest carbonate bed } \\
\sim 7 m \text { downstream from a } \\
\text { prominent point. }\end{array}$ & 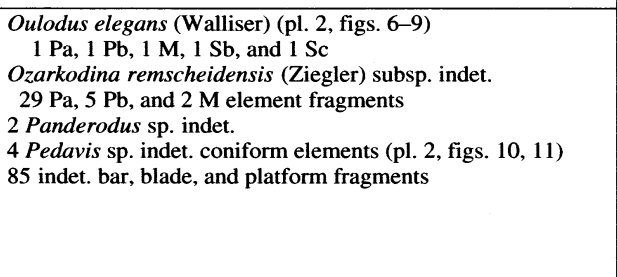 & $\begin{array}{l}\text { late Ludlow into late } \\
\text { Pridoli (from at least } \\
\text { snajdri Zone into late } \\
\text { Pridolian). }\end{array}$ & $\begin{array}{l}\text { Ozarkodinid } \\
\text { biofacies; normal- } \\
\text { marine, shelf } \\
\text { depositional } \\
\text { environnent. } \\
\text { Condition of } \\
\text { conodonts indicates } \\
\text { relatively high- } \\
\text { energy depositional } \\
\text { regime. }\end{array}$ & $\begin{array}{l}\text { Chiefly } \\
5.5 \text { and } \\
\text { minor } 5 \\
\text { and } 6\end{array}$ & $\begin{array}{l}8.5 \mathrm{~kg} \text { processed; } 1.56 \mathrm{~kg} \\
+20 \text { mesh and } 973 \mathrm{~g} \mathrm{~g} \\
20-200 \text { mesh insoluble } \\
\text { residue. Heavy-mineral } \\
\text { concentrate: chiefly } \\
\text { ferruginous siltstone and } \\
\text { detrital mica. }\end{array}$ \\
\hline 11 & $\begin{array}{l}\text { 95MDw216B } \\
\text { (12523-SD) }\end{array}$ & $\begin{array}{l}\text { Somerset } \\
\text { Jackman } \\
45^{3} 383232 " I \\
70^{\circ} 20^{\prime} 50^{\prime \prime}\end{array}$ & $\begin{array}{l}\text { Hardwood Mountain } \\
\text { Formation from outlet } \\
\text { stream of Sugar Bertg } \\
\text { Pond, near Little Big } \\
\text { Wood Pond. Rubble crop } \\
\text { of coral-bearing limestone. }\end{array}$ & 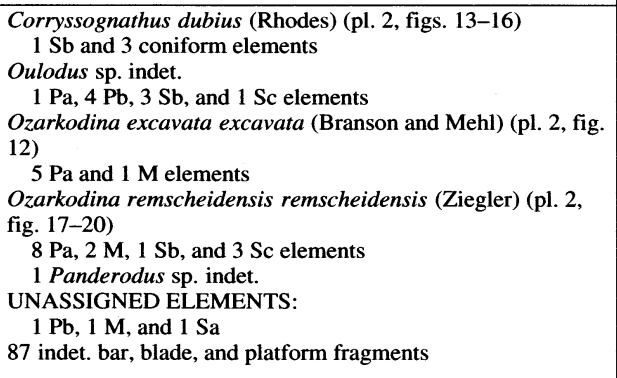 & $\begin{array}{l}\text { late Ludlow (late } \\
\text { Gorstian through } \\
\text { Ludfordian). } \\
\text { Corryssognathus } \\
\text { dubius is the most } \\
\text { biostratigraphically } \\
\text { diagnostic species and } \\
\text { is restricted to the late } \\
\text { Ludlow. }\end{array}$ & $\begin{array}{l}\text { Ozarkodinid- } \\
\text { oulodid; relatively } \\
\text { shallow-water, } \\
\text { moderate- to high- } \\
\text { energy shelf } \\
\text { depositional setting. }\end{array}$ & 5 & $\begin{array}{l}16.6 \mathrm{~kg} \text { processed; } 100 \mathrm{~g} \\
+20 \text { mesh and } 126 \mathrm{~g} \\
20-200 \text { mesh insoluble } \\
\text { residue. Heavy-mineral } \\
\text { concentrate includes rare } \\
\text { subrounded to euhedral } \\
\text { zircon. }\end{array}$ \\
\hline 11 & $\begin{array}{l}\text { 95MDw217A } \\
\text { (12524-SD) }\end{array}$ & $\begin{array}{l}\text { Somerset } \\
\text { Jackman } \\
45^{3} 38355^{\prime \prime \prime} \\
70^{\circ} 20^{\prime} 58^{\prime \prime}\end{array}$ & $\begin{array}{l}\text { Hardwood Mountain } \\
\text { Formation from Sugar } \\
\text { Berth Pond. Shaly } \\
\text { limestone, 5 above } \\
\text { 95MDw216B and } \\
\text { youngest sample collected } \\
\text { at } 5 \text { MDDw216 or } \\
\text { 95MD217. }\end{array}$ & $\begin{array}{l}2 \text { Sb elements } \text { Oulodus sp. indet. } \\
2 \text { Pa elements } \text { Ozarkodina confluens Branson and Mehl (pl. 2, } \\
\text { fig. } 21) \\
\text { Ozarkodina remscheidensis remscheidensis (Ziegler) } \\
8 \text { P Pa, } 1 \text { Pr, and } 1 \text { Sb robust elements } \\
27 \text { Panderodus sp. indet. } \\
27 \text { indet. bar, blade, and platform fragments }\end{array}$ & late Ludlow-Pridoli. & $\begin{array}{l}\text { Indeterminate- } \\
\text { too few conodonts. } \\
\text { Normal-marine, } \\
\text { relatively high } \\
\text { energy depositional } \\
\text { environment. }\end{array}$ & 5 & $\begin{array}{l}24.0 \mathrm{~kg} \text { processed; } 3.97 \mathrm{~kg} \\
+20 \text { mesh and } 220 \mathrm{~g} \\
20-200 \text { mesh insoluble } \\
\text { residue. Heavy-mineral } \\
\text { concentrate: chiefly } \\
\text { ferruginous micaceous and } \\
\text { argillaceous flakes and } \\
\text { common phosphatic } \\
\text { bryozoan pearls. }\end{array}$ \\
\hline 11 & $\begin{array}{l}\text { 95MDw217B } \\
\text { (12525-SD) }\end{array}$ & $\begin{array}{l}\text { Somerset } \\
\text { Jackman } \\
45^{3} 3835^{\prime \prime \prime} \\
70^{3} 20^{\prime \prime} 58^{\prime \prime}\end{array}$ & $\begin{array}{l}\text { Hardwood Mountain } \\
\text { Formation near Little Big } \\
\text { Wood Pond. Shaly } \\
\text { limestone } 10 \mathrm{~m} \text { below } \\
\text { 95MDw216B. }\end{array}$ & $\begin{array}{l}\text { Oulodus sp. indet. } \\
1 \text { Pa, } 1 \text { Pb, } 1 \mathrm{M}, 1 \mathrm{Sb} \text {, and } 1 \mathrm{Sc} \text { elements } \\
\text { Ozarkodian remscheidensis remschieidensis (Ziegler) } \\
12 \mathrm{~Pa}, 5 \mathrm{~Pb} \text {, and } 1 \mathrm{M} \text { elements (mostly fragments) } \\
\text { UNASSIGGED ELMENTS: } \\
1 \mathrm{~Pb}, 1 \mathrm{Sb} \text {, and } 1 \mathrm{Sc} \text { Sc } \\
21 \text { indet. bar, blade, and platform fragments }\end{array}$ & $\begin{array}{l}\text { late Ludlow-Pridoli; if } \\
95 \mathrm{MDW} 217 \mathrm{~B} \text { is } \\
\text { indeed stratigraph- } \\
\text { ically below } 216 \mathrm{~B}, \\
\text { then the age of } 217 \mathrm{~B} \text { is } \\
\text { late Ludlow. }\end{array}$ & $\begin{array}{l}\text { Ozarkodinid; } \\
\text { normal-marine shelf } \\
\text { depositional setting. }\end{array}$ & 5 & $\begin{array}{l}16.9 \mathrm{~kg} \text { processed; } 2.36 \mathrm{~kg} \\
+20 \text { mesh and } 202 \mathrm{~g} \\
20-200 \text { mesh insoluble } \\
\text { residue. }\end{array}$ \\
\hline
\end{tabular}




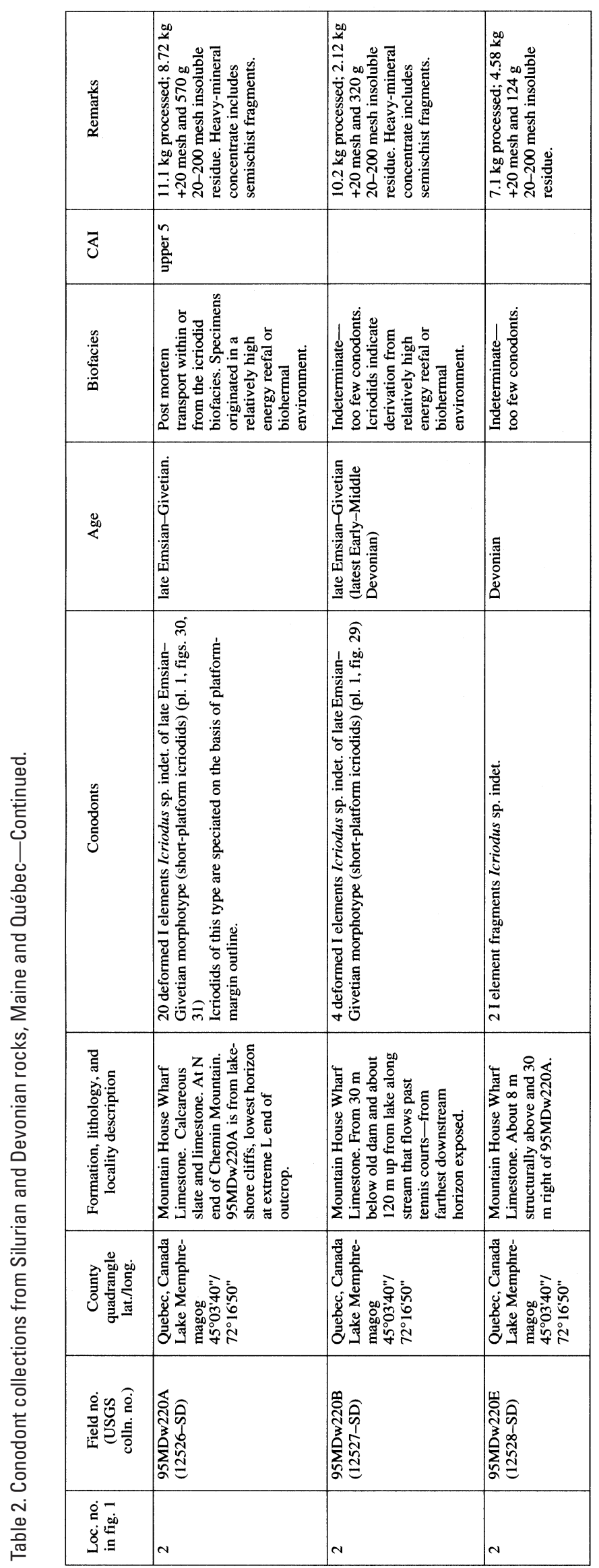

nus is gradationally overlain by siltstone and andesite (West Branch Formation), followed by red shale (Frost Pond Shale); these units are barren of fossils but are probably Lochkovian. The overlying Seboomook Group, which begins the Acadianderived clastic succession, consists of thin-bedded turbidites that have yielded Oriskany-age (Pragian) brachiopods from a locality at Chesuncook Lake (R.B. Neumann, in Griscom, 1976, p. 147-148). The Seboomook Group is overlain progradationally by the Matamagon Sandstone, which has also yielded Pragian brachiopods (for example, R.B. Neumann, in Griscom, 1976, p. 151-152). North of the Katahdin pluton (loc. 17, figs. 1, 4), the Seboomook and Matagamon together compose a prodelta and delta deposited by west-directed paleocurrents (Hall and others, 1976; Pollock and others, 1988). The Matagamon Sandstone is overlain conformably by the Traveler Rhyolite. There appears to have been no hiatus between the two units because clastic dikes of Matagamon Sandstone intrude upward into the basal rhyolite (Rankin, 1968). Concordant U-Pb zircon ages of $406 \mathrm{Ma}$ from the Black Cat Member and 407 Ma from the Pogy Member (Rankin and Tucker, 1995) indicate an early Emsian age for the Traveler Rhyolite and, most likely as well, for the uppermost beds of the Matagamon Sandstone. The Silurian through upper Emsian strata were involved in the regional Acadian folding, which produced a regional northeast-striking cleavage.

The Traveler Rhyolite is overlain by essentially undeformed nonmarine sandstone and conglomerate of the Trout Valley Formation. Although an angular unconformity cannot be demonstrated at outcrop, 1:62,500-scale mapping shows that the Trout Valley Formation truncates major structures in the Traveler (Rankin, 1968). Kasper and others (1988) reviewed the history of age assignments for the Trout Valley on the basis of plant fossils. Palynomorphs from the Trout Valley (McGregor, 1992) indicate a latest Emsian or earliest Eifelian age, corresponding to the douglastownense-eurypterota spore assemblage zone or the patulus and partitus conodont zones (table 1). The Trout Valley is, therefore, significantly younger than the Traveler Rhyolite.

\section{Munsungun Anticlinorium, Maine}

The Munsungun anticlinorium is cored by Ordovician and older rocks, which are unconformably overlain by a complex assortment of locally derived clastic, volcanic, and carbonate rocks (loc. 18, figs. 1, 4). Hall (1970) assigned these rocks to the East Branch Group and to the laterally equivalent Spider Lake Formation. Corals and brachiopods from several fossil occurrences give ages of Ludlow, Pridoli, and "middle or upper Helderberg" (Hall, 1970, p. 35). Next follows the Seboomook Group, the youngest unit in the area, which has yielded brachiopods of Oriskany-age (Pragian) age from three places (loc. 18, figs. 1, 4; Hall, 1970, p. 41). Thus, Acadian deformation must be Emsian or younger along the Munsungun anticlinorium.

A similar succession has been reported from the Millimagassett Lake area, at the south end of the Munsungun anticlinorium (loc. 20, figs. 1, 4). Here, the Grand Lake Seboeis 
Group consists of slate, sandstone, conglomerate, calcareous slate, and volcanic rocks. Hibbard (1994, p. 216) cited nine fossil collections that give a range of "Late Silurian to earliest Devonian." (No details were published). The Grand Lake Seboeis Group grades up into, but may interfinger with, the Millimagassett Lake Formation, which has yielded Late Silurian, most likely Pridoli, brachiopods and corals near its base (Hibbard, 1993); presumably, its upper part extends at least into the Lochkovian, if not the Pragian. According to Hibbard (1994), the Millimagassett Lake Formation is a flysch succession resembling the Seboomook Group. It appears to be the oldest such flysch yet identified along the anticlinorial belt, possibly 2 to 3 m.y. older than the Seboomook Group at Beck Pond (loc. 12, figs. 1, 4) and 5 to 10 m.y. older than along the Munsungun anticlinorium (loc. 18, figs. 1, 4). In the Millimagassett Lake area, Hibbard (1994) reported evidence for an early Acadian sinistral shear deformation that was followed by the main, regional Acadian folding on northeasttrending axes. Both phases of deformation are Lochkovian or younger.

\section{Pennington Anticlinorium, Maine}

To determine the timing of Acadian foreland-basin sedimentation and deformation along the northwestern limb of the Pennington anticlinorium (locs. 21-23, figs. 1, 4), we must first unravel some stratigraphic problems. The Silurian to Devonian Fish River Lake Formation of Boone (1970) consists of sandstone, siltstone, conglomerate, mafic and silicic volcanic rocks, and impure limestone. Unnamed unit DSus, as shown on the bedrock geologic map of Maine (Osberg and others, 1985), occurs along strike from the Fish River Lake Formation at the same broad stratigraphic level. New information reported here suggests that both the Fish River Lake and unit DSus are made up of two unrelated successions: an older one that includes volcanic and calcareous rocks, and a younger one composed of plant-bearing siltstone, sandstone, and conglomerate.

Carbonate rocks from the Fish River Lake Formation have yielded brachiopods of Late Silurian ("Cobleskill," Pridoli) and Early Devonian ("New Scotland," Lochkovian) age (A.J. Boucot, in Boone, 1970, p. 33-34). We processed a shaly limestone from one of Boucot's Cobleskill-age locations (loc. 21, figs. 1, 4) for conodonts, but the sample yielded only nondiagnostic Silurian-Devonian forms (table 2). Within unit DSus, the informally named Square Lake Limestone occurs in a single lakeshore exposure (loc. 23, figs. 1, 4). A.J. Boucot (in Boone, 1970) placed the brachiopod and coral fauna "definitely within the lower Helderberg series" (that is, woschmidti Zone to at least eurekaensis Zone of the Lochkovian). Conodonts from the most diagnostic of two samples of reef limestone give an age range of late Ludlow to early Lochkovian (pl. 2, figs. 22-32, 36-38; loc. 23, figs. 1, 4; table 2).

Plant-bearing sandstone and siltstone from the Fish River Lake Formation of Boone (1970) and unit DSus have yielded spores, some of which are considerably younger than the brachchiopods (table 1; McGregor, 1962, 1963, 1968, 1996) This conclusion is based on material submitted (without lithologic descriptions) to D.C. McGregor by Eli Mencher in the 1960's and by D.C. Bradley in 1995. In unit DSus, the most closely dated of two siltstone samples is clearly Early Devonian, probably Lochkovian or early Pragian (table 1). Bedding and a strong cleavage (presumably Acadian) in these rocks both are subvertical. In the Fish River Lake Formation, two samples of dark, thin-bedded siltstone are of late Pragian-early Emsian age (polygonalis-emsiensis Zone). These rocks, which resemble the Seboomook Group, are also folded and cleaved. Three sandstone samples from 1995 and two samples of unknown lithology from the 1960's are of late Emsian or early Eifelian age (douglastownense-eurypterota Zone). The 1995 samples of this age are from a succession of fluvial or marginal-marine plant-bearing sandstone and conglomerate at Nadeau Thoroughfare (loc. 22 , figs. 1,4$)$, which dip moderately $\left(50^{\circ}\right)$ but lack cleavage. Finally, one sample of unknown lithology submitted to McGregor by Mencher is early or middle Eifelian (velata-langii Zone).

Piecing together these various facts, we suggest that farforeland carbonate sedimentation was underway during the Late Silurian and at least part of the Lochkovian. An influx of orogenically derived siliciclastic materials began during late Lochkovian or early Pragian time. Deposition of the plant-bearing sandstone probably lasted from about late Emsian into the early or middle Eifelian. Taken together, the palyniferous strata can be interpreted as components of a prograding foreland-basin sequence, akin to the somewhat older SeboomookMatagamon succession farther south.

\section{Madawaska Area, New Brunswick}

Two useful constraints on the date of the Acadian orogeny are available from northwesternmost New Brunswick, where the Aroostook-Matapedia and Connecticut Valley-Gaspé Basins come together (loc. 24, figs. 1, 4). The Upper Ordovician and Silurian are represented by various deep-water facies (St. Peter, 1977). The youngest Silurian rocks are mostly green slate and calcareous siltstone of the Perham Formation, which has yielded graptolites as young as middle to late Ludlow (St. Peter, 1977, p. 30). There follows a gap in the fossil record spanning the Pridoli, Lochkovian, and Pragian. The overlying Temiscouata Formation consists of several kilometers of siliciclastic turbidites characterized by abundant slump folds; Hesse and Dalton (1995) interpreted the Temiscouata as a submarine foreland-basin succession. It has yielded brachiopods now assigned an Emsian age (St. Peter and Boucot, 1981, p. 91). The Temiscouata Formation has been penetratively deformed; Acadian deformation must be Emsian or younger in age.

\section{LaPatrie and Ste.-Cécile de Whitton, Eastern Townships, Québec}

In the Eastern Townships of Québec, the axial region of the Connecticut Valley-Gaspé Basin is occupied by a thick succession of Acadian-deformed sandstone and slate assigned to the Compton Formation (Slivitski and St.-Julien, 1987). Sandstone from the Compton Formation has yielded Emsian 
plant fossils (from locs. 3, 4, figs. 1, 4; Hueber and others, 1990). Although the age of onset of foreland-basin sedimentation in this area is unknown, the presence of Emsian forelandbasin deposits suggests that the orogen was not far off at the time. Acadian deformation in the Eastern Townships must have taken place between 409-394 Ma (possible age range of the Compton Formation) and the 384 2 2-Ma Scotstown pluton, which is the oldest postfolding pluton that intrudes the Compton Formation (Simonetti and Doig, 1990).

\section{Northwestern Margin of the Connecticut Valley-Gaspé Basin, Québec}

Three localities along the northwestern margin of the Connecticut Valley-Gaspé Basin in Québec provide important constraints on the age of Acadian foreland-basin sedimentation and deformation. The most informative strata are at St.-Georges (loc. 5, figs. 1, 4), where the Eifelian Famine Formation unconformably overlies the Ordovician Magog Group (Uyeno and Lespérance, 1997). The Famine begins with a basal conglomerate, overlain by limestone, which is coral bearing at the bottom but becomes progressively shalier upsection. The maximum preserved thickness is $215 \mathrm{~m}$. Uyeno and Lesperance (1997) documented a conodont fauna of costatus Zone age, a refinement on the previously quoted Eifelian age (Oliver, 1971), which was based on corals and brachiopods. The Famine Formation is the youngest unit at St.-Georges; it was involved in regional Acadian deformation, which thus must postdate the costatus Zone.

In the Lac Temiscouata area (loc. 7, figs. 1, 4), the situation is similar to that at St.-Georges. The key unit, comparable to the Famine, is the Touladi Limestone, which rests with slight angular unconformity on various older units ranging in age from possibly Ordovician to Late Silurian (Uyeno and Lespérance, 1997). The Touladi consists of mostly bioclastic limestone, with a maximum preserved thickness of $105 \mathrm{~m}$. Recent conodont studies by Uyeno and Lespérance (1997) indicate a position in either the australis or kockelianus Zone of the Eifelian, an assignment that refines but does not significantly alter the Eifelian age quoted by Boucot and Drapeau (1968).

Devonian limestone at Lac Memphremagog (loc. 2, fig. 1) suggests a similar interpretation, although biostratigraphic control is not so tight. There, the Mountain House Wharf Limestone is a dark, slaty limestone surrounded by, and probably faulted against, Ordovician rocks (Boucot and Drapeau, 1968). It has yielded a brachiopod and coral fauna of Eifelian age (Boucot and Drapeau, 1968, p. 9). Three of four large conodont samples yielded only poorly preserved forms that could be assigned only a middle Emsian to Givetian age (table 2).

The coral-bearing shallow-water limestone at St.Georges, Lac Temiscouata, and Lac Memphremagog was probably deposited in a far-foreland setting. The upwarddeepening trend at St.-Georges most likely records the beginnings of foreland-basin subsidence. Reckoning that an interval of foreland-basin sedimentation would have preceded deformation, the Acadian orogeny most likely took place at St.-
Georges during the Givetian or, conceivably, the late Eifelian. Deformation at Lac Temiscouata probably took place a few million years later than at St.-Georges.

\section{Montreal, Québec}

Devonian strata do not crop out anywhere north of the Mohawk River in central New York but were once present in Montreal, $330 \mathrm{~km}$ to the north (loc. 1, figs. 1, 4). The Ste. Hélène Island breccia (Boucot and others, 1986) is a Cretaceous diatreme that was emplaced into a region of gentle foreland deformation about $20 \mathrm{~km}$ cratonward of the Paleozoic thrust front. The diatreme contains an assortment of xenoliths that reveal the former existence of a Devonian succession similar to that in eastern and central New York State. This diatreme, in the words of J.B. Thompson, is a "drill hole in the sky." The inferred sequence is Lochkovian (Helderbergian) limestone, Pragian (Oriskany-age) sandstone and limestone, Emsian (Bois Blanc or Schoharie age) limestone, Eifelian (early and late Onondagan) limestone, and Givetian (Hamilton-age) micritic limestone, siltstone, and sandstone. The latter siltstone and sandstone resemble the Hamilton, which in the Catskills of eastern New York State constitutes the base of the main foreland-basin sequence. By implication, the leading edge of the foreland basin was near Montreal during Givetian time.

\section{Isotopic Ages of Acadian Plutons}

When the present study was first conceived, only a few high-precision $\mathrm{U}-\mathrm{Pb}$ and ${ }^{40} \mathrm{Ar} /{ }^{39} \mathrm{Ar}$ ages were available for Silurian and Devonian plutons in the study area (fig. 1) - not enough to reveal a clear regional age pattern for the plutons. Accordingly, we set out to date all the significant Acadian plutons in a swath from the Maine coast to the MaineQuébec border. New U-Pb and (or) ${ }^{40} \mathrm{Ar} /{ }^{39} \mathrm{Ar}$ ages are reported here for 17 plutons. Other new ages from the geochronology laboratory of Washington University, St. Louis, were reported by Tucker and others (in press) for plutons near and on the Maine coast, by Rankin and Tucker (1995) for the Katahdin area, by Solar and others (1998) for western interior Maine, and by Eusden and others (in press) for northern New Hampshire. Figure 8 and table 3, which summarize the best available emplacement ages, clearly reveal plutonic belts of about 420 Ma near the coast and 407-406 Ma farther inland, which are essential in positioning the deformation front. Other plutons are significantly younger than about $406 \mathrm{Ma}$, some of which also occur in discrete belts, but they are not so useful in tracking the Acadian front.

Acadian plutons fall into three broad and somewhat overlapping categories, pretectonic, syntectonic, and posttectonic, with respect to the local first phase (D1) of Acadian deformation. A pluton might be classified as posttectonic, even though the Acadian orogeny was still underway elsewhere during the pluton's emplacement. We considered three types of anecdotal evidence in assessing the age of plutonism relative to Acadian 
deformation: (1) map-scale relations to Acadian folds and faults, (2) plutonic-rock fabrics, and (3) metamorphic textures in the contact aureole.

\section{U-Pb Analytical Methods}

$\mathrm{U}-\mathrm{Pb}$ analyses (fig. 7; table 4) were performed at the geochronology laboratory of Washington University, St. Louis. Zircon was extracted from 5- to 10-kg samples, using standard techniques of density and magnetic separation. Zircon grains were selected for analysis on the basis of size, color, clarity, and morphology. Sample sizes varied, depending on U content, grain size, and age, but most analyses were performed on fractions of 5 to 20 crystals, generally with less than 200 ng of radiogenic $\mathrm{Pb}$. All analyses were air abraded (Krogh, 1982) and cleaned sequentially in warm $4 \mathrm{~N} \mathrm{HNO}_{3}$, water, and distilled acetone to remove components carrying common $\mathrm{Pb}$. Washed and weighed zircon fractions were then loaded in Teflon bombs, spiked with a mixed ${ }^{205} \mathrm{~Pb}-{ }^{235} \mathrm{U}$ tracer solution, and digested in 48 percent $\mathrm{HF}$ and $7 \mathrm{~N} \mathrm{HNO}_{3}$ for 72 hours at $210^{\circ} \mathrm{C}$. After digestion and conversion to chloride form, $\mathrm{Pb}$ and $\mathrm{U}$ were purified by using ion-exchange techniques, as described in Krogh (1973). Total-procedure blanks for $\mathrm{Pb}$ and $\mathrm{U}$, measured during the period of analysis, were 1-8 and less than $1 \mathrm{pg}$, respectively; total common- $\mathrm{Pb}$ abundances are reported for each analysis in table 4 . Initial- $\mathrm{Pb}$ compositions result in insignificant changes to the calculated ages.

Isotopic-ratio measurements of $\mathrm{Pb}$ and $\mathrm{U}$ were made in a VG Sector 54 thermal-ionization mass spectrometer with enhanced pumping capacity, seven movable collectors, and a Daly-type detector with ion-counting capability. Lead and uranium were loaded together on outgassed single-Re filaments with silica gel and phosphoric acid, and all measurements were made by the method of peak hopping in ion-counting mode. Ion-beam intensities ranged from $0.5 \times 10^{-13}$ to $1.5 \times 10^{-13} \mathrm{~A}$ for ${ }^{206} \mathrm{~Pb}^{+}$and from $0.5 \times 10^{-13}$ to $2.5 \times 10^{-13} \mathrm{~A}$ for ${ }^{235} \mathrm{U}$ (measured as $\mathrm{UO}_{2}^{+}$). Daly bias and nonlinearity were periodically monitored with U.S. National Institute of Standards \& Technology and Central Bureau for Nuclear Measurements isotopic reference materials, and correction factors and errors for Daly gain were used in data reduction.

Errors for the ${ }^{238} \mathrm{U} /{ }^{206} \mathrm{~Pb},{ }^{235} \mathrm{U} /{ }^{207} \mathrm{~Pb}$, and ${ }^{207} \mathrm{~Pb} /{ }^{206} \mathrm{~Pb}$ ages were estimated by using the method of Ludwig (1980); all age uncertainties are quoted at the 95-percent-confidence level. Cited ages are the mean ${ }^{207} \mathrm{~Pb} /{ }^{206} \mathrm{~Pb}$ age of concordant or slightly discordant analyses weighted according to the inverse variance of each analysis (Ludwig, 1992); the quoted age error is the standard error of the average value calculated by using the assigned error for each analysis. The reliability of all cited ages may be evaluated by the mean square of weighted deviates (MSWD), which is a measure of the observed scatter to that predicted by the assigned errors to each analysis. For all samples, the MSWD is much less than 1, indicating that assigned errors may be overestimated.

\section{${ }^{40} \mathrm{Ar} /{ }^{39} \mathrm{Ar}$ Analytical Methods}

${ }^{40} \mathrm{Ar} /{ }^{39} \mathrm{Ar}$ analyses (fig. 8; table 5) were performed at the geochronology laboratory at the University of Maine, Orono. Samples were separated by using standard magnetic and density separation techniques. The purity of the samples was estimated to be greater than 99.5 percent. Samples, flux monitors (interlaboratory standard SBG-7), and K and Ca salts were encapsulated in $\mathrm{Al}$ foil and sealed in silica glass vials. These vials were irradiated in the L67 position of the Ford Nuclear Reactor at the University of Michigan. Micas and flux monitors weighed approximately $35 \mathrm{mg}$. Samples were heated in a molybdenum crucible within the ultra-high-vacuum system on line to the mass spectrometer using radio-frequency induction. Temperatures have an estimated uncertainty of $\pm 50^{\circ} \mathrm{C}$. Inert gases were purified by using standard gettering techniques. The Ar-isotopic composition was measured digitally, using a Nuclide 6-60-SGA 1.25 mass spectrometer. All data were corrected for mass discrimination and interfering $\mathrm{Ar}$ isotopes produced during irradiation (Dalrymple and others, 1981). The decay constants recommended by Steiger and Jäger (1977) were used to calculate the ages. Error calculations, which included both the uncertainty in the analytical measurement and the uncertainty in the $J$ value, are reported at the $2 \sigma$ level. Each sample was analyzed in a minimum of 8 and no more than 15 increments. A plateau age represents the mean of ages in consecutive increments that do not differ on the basis of $2 \sigma$ analytical uncertainties.

\section{Ludlow, Pridoli, and Lochkovian Plutons}

Until a few years ago, the numerous gabbroic to granitic plutons along the Maine coast were all assigned to the Devonian, but in light of modern ${ }^{40} \mathrm{Ar} /{ }^{39} \mathrm{Ar}$ and $\mathrm{U}-\mathrm{Pb}$ dating and the new time scale, the oldest group of plutons now is known to range from Ludlow to Lochkovian. The first pluton in Maine to yield an undoubted Silurian age was the Pocomoonshine gabbrodiorite (PO, fig. 6), which yielded an ${ }^{40} \mathrm{Ar} /{ }^{39} \mathrm{Ar}$ amphibole plateau age of $422.7 \pm 3 \mathrm{Ma}$ (West and others, 1992). The pluton is posttectonic: it intruded already-deformed turbidites of the presumably Lower Silurian Flume Ridge Formation and "stitches" the fault contact between the Fredericton Basin and the St. Croix belt to the south (West and others, 1992). New zircon ages from the Utopia (423.0 $1.0 \mathrm{Ma}$; UT, fig.

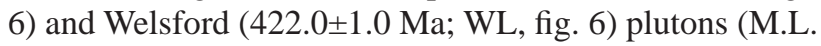
Bevier, written commun., 1996) extend the belt of known Ludlow plutons into southern New Brunswick. Several plutons in the Penobscot Bay area have also yielded concordant zircon ages of 424 to $417 \mathrm{Ma}$ - that is, Late Silurian to earliest Devonian-including the Somesville $(424.0 \pm 2.0 \mathrm{Ma}$; SV, fig. 6), Blinn Hill (424.0 $\pm 2.0 \mathrm{Ma}$; BH, fig. 6), Lake St. George

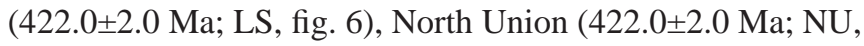

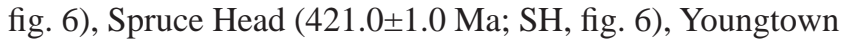

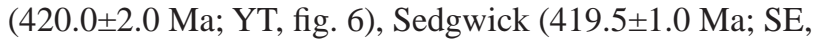
fig. 6), South Penobscot (419.2 \pm 2.2 Ma; SP, fig. 6), Cadillac

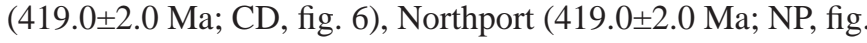

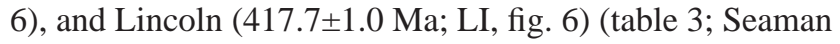


and others, 1995; Stewart and others, 1995b; Tucker and others, in press) plutons. The North Union and Lake St. George plutons are composed of granitic gneiss inferred to have been emplaced syntectonically (Tucker and others, in press). West and others (1995) reported widespread Late Silurian to earliest Devonian ${ }^{40} \mathrm{Ar} /{ }^{39} \mathrm{Ar}$ ages from metamorphic hornblende outboard of the Sennebec Pond Fault, essentially coeval with the plutons listed above.

\section{Early Emsian Plutons}

\section{Previous Results}

Nearly two dozen closely dated plutons of Emsian age define a northeast-southwest belt that can be traced across the entire strike length of figure 6 and beyond, into Massachusetts. Previously, there was some indication of plutonic activity at about 400 to $410 \mathrm{Ma}$ along this trend, but little

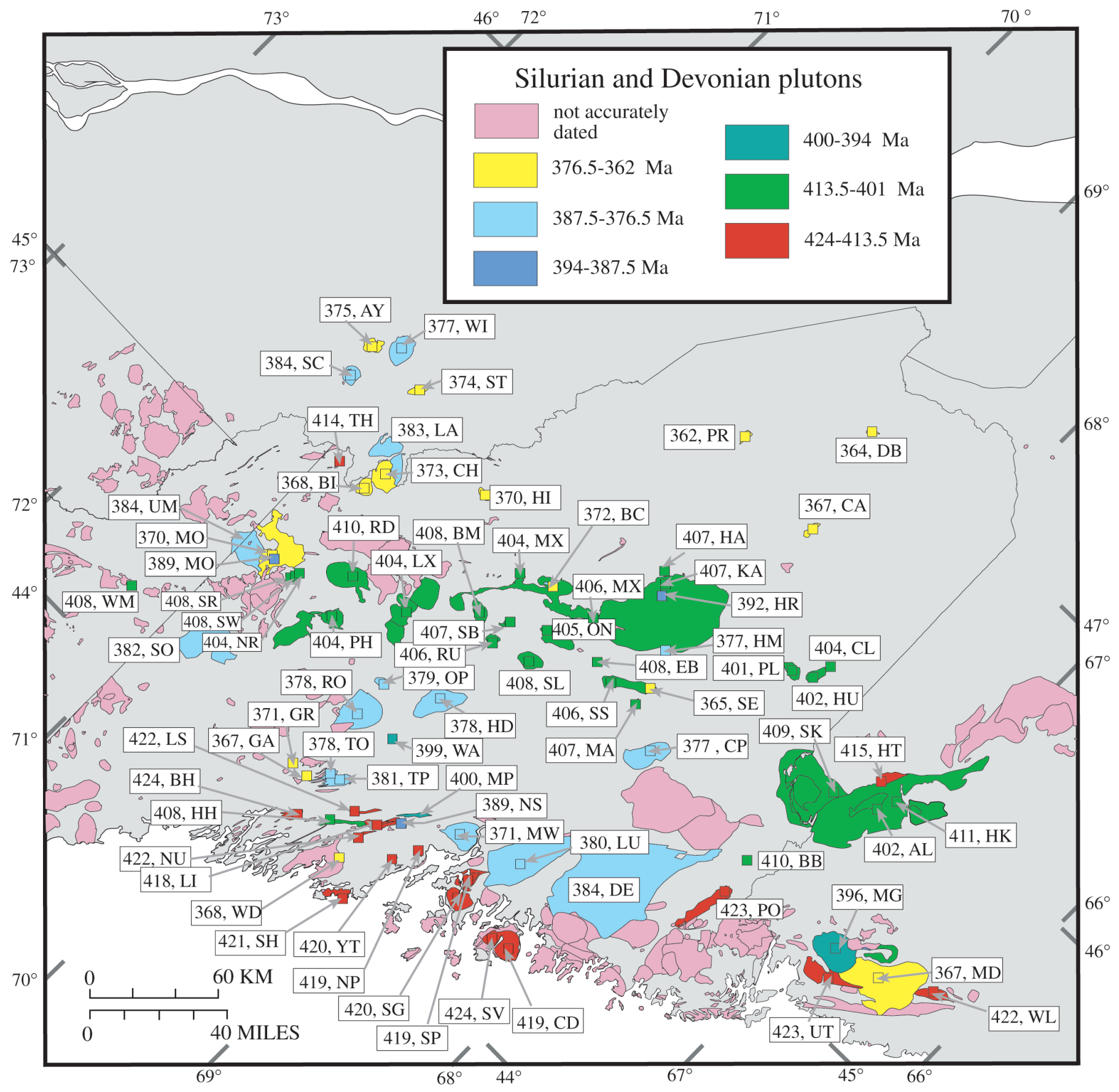

Figure 6.-Maine and adjacent parts of New Brunswick, Québec, New Hampshire, and Vermont, showing distribution of Late Silurian, Devonian, and earliest Carboniferous plutons. Boxes show best estimate of intrusive age (in $\mathrm{Ma}$; concordant $\mathrm{U}-\mathrm{Pb}$ or ${ }^{40} \mathrm{Ar} /{ }^{39} \mathrm{Ar}$ plateau) and two-letter abbreviation, keyed to table 3. 


\section{EMSIAN INTRUSIONS}
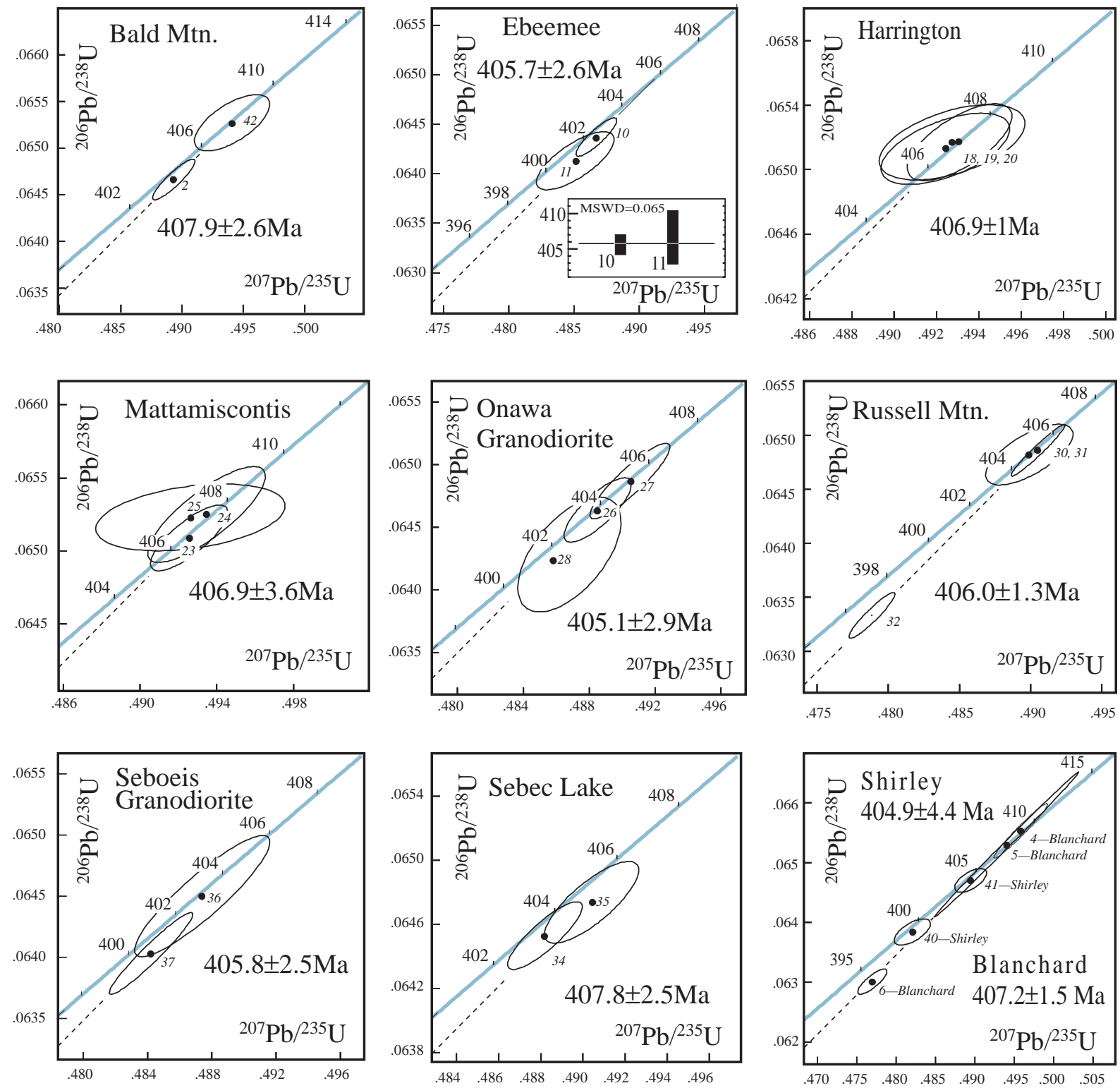

FRASNIAN AND FAMENNIAN INTRUSIONS
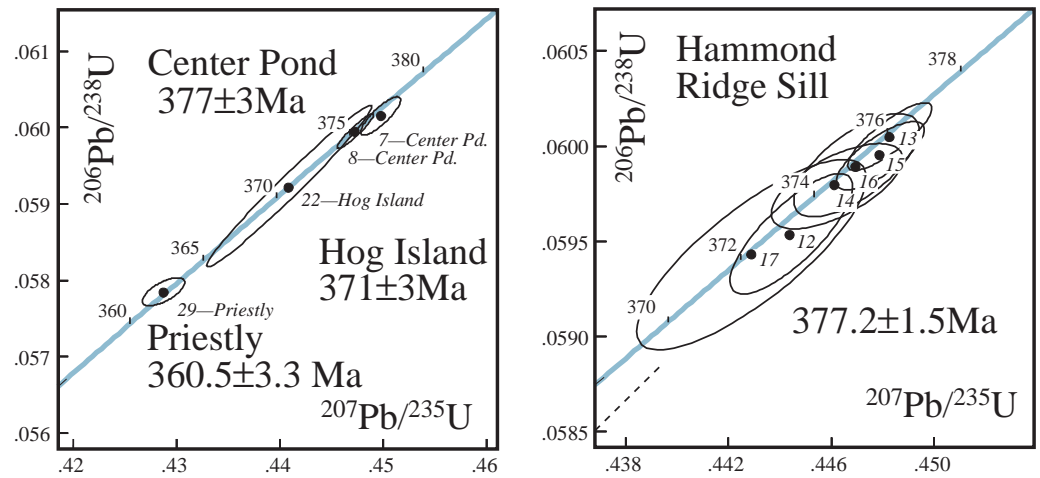

Figure 7.- U-Pb concordia diagrams of intrusive rocks dated in this report. 
evidence to suggest that most plutons along the belt would turn out to fall within the same narrow age range. In western New Brunswick, Bevier and Whalen (1990a) reported U-Pb ages of $409 \pm 2 \mathrm{Ma}$ for the Skiff Lake phase of the Pokiok batholith and 402 $\pm 1 \mathrm{Ma}$ for the Allandale phase (SK and AL, respectively, fig. 6). In eastern Maine, Hubacher and Lux (1987) obtained ${ }^{40} \mathrm{Ar} /{ }^{39} \mathrm{Ar}$ ages, which are now known to be Emsian, from three plutons that truncate regional Acadian structures: the Pleasant Lake (400.5 $\pm 4.5 \mathrm{Ma}$; PL, fig. 6), Hunt Ridge (401.5 \pm 4 Ma; HU, fig. 6), and Cochrane Lake

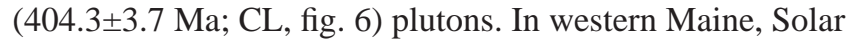
and others (1998) recently reported Emsian U-Pb ages from the South Roxbury granite (408.2 $\pm 2.5 \mathrm{Ma}$; SR, fig. 6), Swift River granite (407.9 \pm 1.9 Ma; SW, fig. 6), Redington pluton (407.6 $\pm 4.6 \mathrm{Ma}$; RD, fig. 6), North Roxbury granite (404.3 $\pm 1.9 \mathrm{Ma}$; NR, fig. 6), Lexington pluton (404.3 \pm 1.8 Ma; LX, fig. 6), and various phases of the Phillips pluton (405.3 $\pm 1.8,403.6 \pm 2.2$, and 403.5 $\pm 1.6 \mathrm{Ma}$; PH, fig. 6). In the Presidential Range of northern New Hampshire, the Wamsutta pluton (WM, fig. 6), which was emplaced during F1 Acadian folding, has yielded a U-Pb zircon age of 408.4 $\pm 1.9 \mathrm{Ma}$ (Eusden and others, in press). In New Hampshire, outside the area of figure 6, Emsian U-Pb ages have been obtained for the Spaulding pluton (408.4 \pm 1.9 Ma on zircon; Robinson and Tucker, 1996), the Ashuelot pluton (403 \pm 3 Ma on monazite; Robinson and Tucker, 1996), and the Cardigan pluton (404 \pm 2 Ma on zircon; R.D. Tucker, unpub. data). Finally, the Prescott gabbro, which postdates the nappe phase of regional Acadian
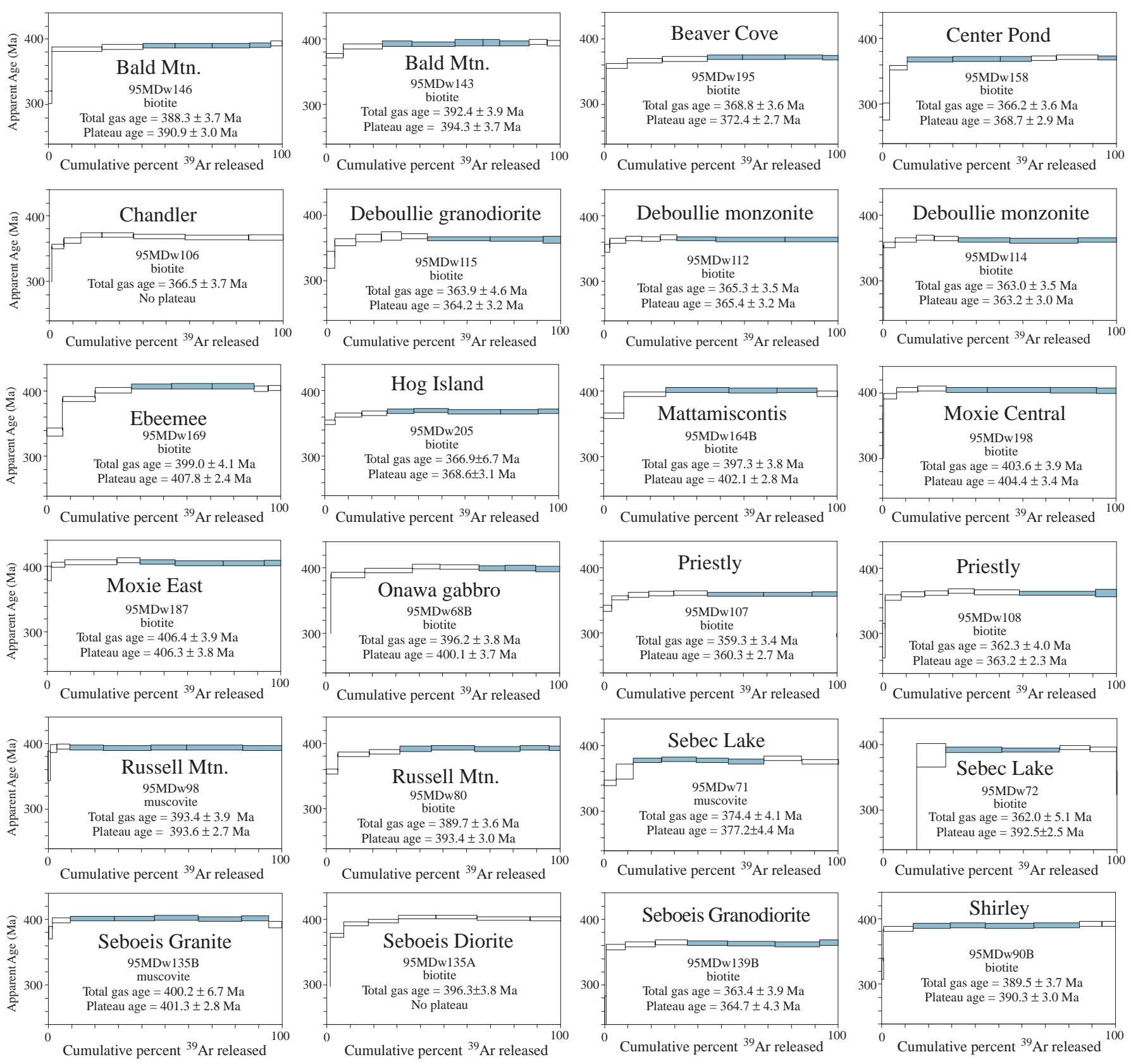
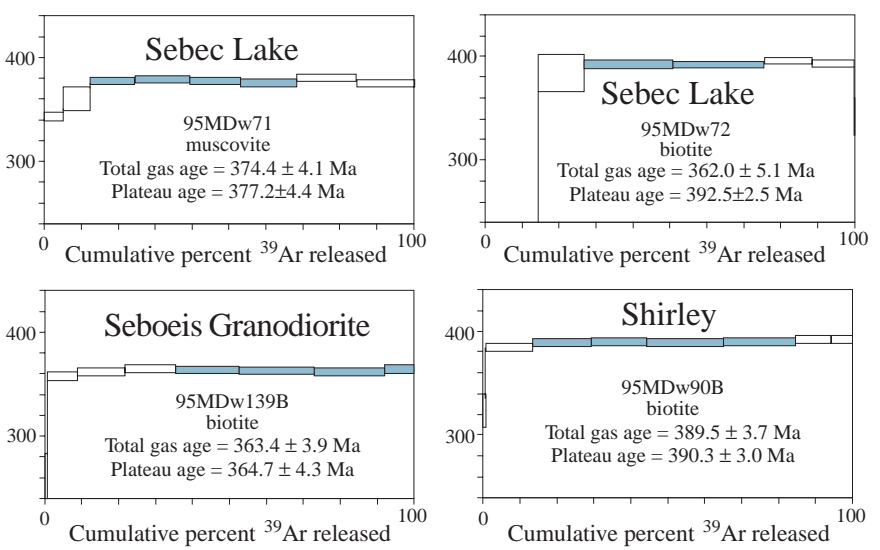

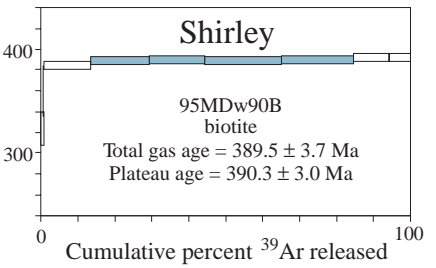

Figure 8. $-{ }^{40} \mathrm{Ar} /{ }^{39} \mathrm{Ar}$ age spectra of intrusive rocks dated in this report. Shaded rectangles correspond to data used to calculate plateau ages. 


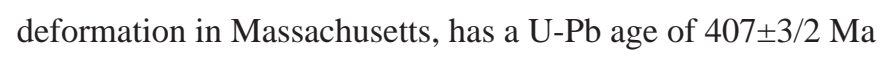
(Tucker and Robinson, 1990). Far outboard of the main belt of Emsian magmatism are two outliers: the Haskell Hill pluton (U-Pb age, 408 $\pm 5 \mathrm{Ma}$; HH, fig. 6; Tucker and others, in press) and the Berry Brook gabbro-diorite $\left({ }^{40} \mathrm{Ar} /{ }^{39} \mathrm{Ar}\right.$ age, $410 \mathrm{Ma}$; BB, fig. 6; Ludman and Idleman, 1998).

\section{Russell Mountain Pluton}

The Russell Mountain pluton (RU, fig. 6) is a unfoliated two-mica granodiorite. Mapping by Ludman (1978) showed that the pluton truncates regional folds in the Madrid and Carrabassett Formations, and so, on this basis, it must postdate at least some Acadian deformation. Contact-metamorphic rocks on the northwestern margin, however, have a schistose foliation and aligned chiastolite with biotite strain shadows (fig. $9 A$ ). Because metamorphic biotite is incipient or absent outside the aureole (Ludman, 1978), some deformation must have taken place while the pluton was being emplaced. Whether deformation in the contact aureole was caused by pluton emplacement or was a regional event is unknown. Two concordant zircon fractions yield a mean ${ }^{207} \mathrm{~Pb} /{ }^{206} \mathrm{~Pb}$ age of 406.0 $\pm 1.3 \mathrm{Ma}$ (fig. 7). Two biotite fractions yielded ${ }^{40} \mathrm{Ar} /{ }^{39} \mathrm{Ar}$

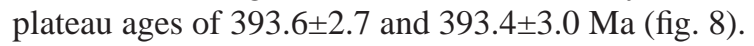

\section{Sebec Lake Pluton}

The Sebec Lake pluton (SL, fig. 6) is a unfoliated biotite granodiorite that, like the Russell Mountain pluton, truncates regional folds involving the Madrid and Carrabassett Formations (Griffin, 1971). On the basis of its map-scale relations, it must postdate at least some Acadian deformation. Contact-metamorphic textures, however, are synkinematic. Metamorphosed Carrabassett Formation in the aureole contains large andalusite porphyroblasts with asymmetric strain shadows made up of biotite and chlorite aligned in the foliation (fig. 9B). Outside the contact aureole, the rocks are at subbiotite grade; thus, the biotite and andalusite are both related to plutonism. Whether deformation in the contact aureole was caused by pluton emplacement or was a regional event is unknown. We obtained two concordant zircon fractions that yielded a mean ${ }^{207} \mathrm{~Pb} /{ }^{206} \mathrm{~Pb}$ age of $407.8 \pm 2.5 \mathrm{Ma}$ (fig. 7) and ${ }^{40} \mathrm{Ar} /{ }^{39} \mathrm{Ar}$ plateau ages of $392.5 \pm 2.5 \mathrm{Ma}$ from biotite and 377.2 \pm 4.4 Ma from muscovite (fig. 8).

\section{Bald Mountain Pluton}

The Bald Mountain pluton (BM, fig. 6) is a unfoliated two-mica granodiorite; in map view, it has the shape of an ellipse with its long axis normal to regional strike. Mapping by Espenshade and Boudette (1967) and Ludman (1978) showed that the pluton truncates regional folds in the Madrid and Carrabassett Formations and on this basis would appear to postdate at least some Acadian deformation. Unoriented chiastolite and biotite porphyroblasts in steeply dipping contact-metamorphosed strata also suggest posttectonic emplacement (fig. 9C). A single concordant zircon fraction indicates an emplacement age near $408 \mathrm{Ma}$ (table 4). ${ }^{40} \mathrm{Ar} /{ }^{39} \mathrm{Ar}$ ages were obtained from three biotite separates, two of which yielded plateaus of $390.9 \pm 3.0$ and $394.3 \pm 3.7 \mathrm{Ma}$, giving a mean age of 392.6 \pm 2 Ma for cooling through the closure temperature of argon in biotite (fig. 8).

\section{Onawa Pluton}

The Onawa pluton (ON, fig. 6) is a zoned body with a granodiorite core and a gabbroic rim. It truncates and deflects the regional structural grain defined by steeply dipping bedding in the Carrabassett Formation. Thin-section observations reported by Van Heteren and Kusky (1994) suggest that andalusite in the contact aureole overgrew a preexisting cleavage; thus, the pluton is posttectonic, at least in part. We obtained three concordant zircon fractions that yielded a mean ${ }^{207} \mathrm{~Pb} /{ }^{206} \mathrm{~Pb}$ age of $405.1 \pm 2.9 \mathrm{Ma}$ for the granodiorite (fig. 9), and an ${ }^{40} \mathrm{Ar} /{ }^{\beta 9} \mathrm{Ar}$ plateau age of $400.1 \pm 3.7 \mathrm{Ma}$ on biotite from the gabbro (fig. 8).

\section{Shirley-Blanchard Pluton}

The Shirley-Blanchard pluton (SB, fig. 6) is a composite pluton that includes a more mafic phase of pyroxene-hornblende granodiorite (Blanchard body) and a more felsic phase of granodiorite (Shirley body; Espenshade and Boudette, 1967). The pluton truncates regional-scale tight to isoclinal folds involving the Madrid and Carrabassett Formations. From the Shirley body, we obtained two concordant zircon analyses (fig. 7) with a mean ${ }^{207} \mathrm{~Pb} /{ }^{206} \mathrm{~Pb}$ age of $404.9 \pm 4.4 \mathrm{Ma}$ and an ${ }^{40} \mathrm{Ar} /{ }^{39} \mathrm{Ar}$ plateau age of 390.3 $\pm 3.0 \mathrm{Ma}$ on biotite (fig. 10). From the Blanchard body, we obtained two concordant zircon fractions (fig. 7) that yielded a mean ${ }^{207} \mathrm{~Pb} /{ }^{206} \mathrm{~Pb}$ age of 407.2 $\pm 1.5 \mathrm{Ma}$. The weighted mean ${ }^{207} \mathrm{~Pb} /{ }^{206} \mathrm{~Pb}$ age of all zircon analyses from the Shirley-Blanchard body is $406.9 \pm 1.4 \mathrm{Ma}$ (fig. 7), our preferred age for this composite pluton.

\section{Mattamiscontis Pluton}

The Mattamiscontis pluton (MA, fig. 6) is an unfoliated biotite granite; fieldwork for this study was limited to geochronologic sampling of the pluton and brief examination of outcrops in the contact aureole. Reconnaissance mapping compiled by Osberg and others (1985) showed the pluton cutting a syncline cored by Carrabassett Formation; similarly, at one outcrop in the contact aureole, tightly folded metapelite displays a weak axial-planar cleavage that is overgrown by cordierite. A thin section from another outcrop, however, shows oriented cordierite surrounded by biotite strain shadows. (The regional metamorphic grade is chlorite.) These observations together suggest that the pluton postdates Acadian folding, but that some deformation-either regional or pluton relatedaccompanied pluton emplacement. A granite sample yielded three concordant zircon analyses with a mean ${ }^{207} \mathrm{~Pb} /{ }^{206} \mathrm{~Pb}$ age of 406.9 $\pm 3.6 \mathrm{Ma}$ (fig. 7) and an ${ }^{40} \mathrm{Ar} /{ }^{39} \mathrm{Ar}$ biotite plateau age of $402.1 \pm 2.8 \mathrm{Ma}$ (fig. 8). 
Table 3. Emplacement ages of plutons mapped in figure 6.

[State or province: ME, Maine; NB, New Brunswick; NH, New Hampshire; QU, Québec; ]

\begin{tabular}{|c|c|c|c|c|c|c|c|c|}
\hline Pluton & $\begin{array}{l}\text { State or } \\
\text { province }\end{array}$ & $\begin{array}{l}\text { Abbrev. } \\
\text { (fig. } 7 \text { ) }\end{array}$ & $\begin{array}{l}\text { Age } \\
\text { (Ma) }\end{array}$ & $\begin{array}{l}\text { Error } \\
\text { (Ma) }\end{array}$ & Method & Mineral & Comments & Reference \\
\hline $\begin{array}{l}\text { Allandale pluton, } \\
\text { Pokiok batholith }\end{array}$ & NB & $\mathrm{AL}$ & 402.0 & 1 & $\mathrm{U}-\mathrm{Pb}$ & Monazite & $\begin{array}{l}\text { Mean }{ }^{235} \mathrm{U} / 207 \mathrm{~Pb} \text { age; } 2 \text { concordant } \\
\text { fractions. }\end{array}$ & $\begin{array}{l}\text { Bevier and Whalen } \\
(1990 \mathrm{a})\end{array}$ \\
\hline Aylmer pluton & QU & AY & 375.0 & 3 & $\mathrm{U}-\mathrm{Pb}$ & Monazite & $\begin{array}{l}{ }^{238} \mathrm{U} / 206 \mathrm{~Pb} \text { age of } 1 \text { nearly concordant } \\
\text { fraction. }\end{array}$ & Simonetti and Doig (1990). \\
\hline $\begin{array}{l}\text { Bald Mountain pluton } \\
\text { Beaver Cove pluton }\end{array}$ & $\begin{array}{l}\text { ME } \\
\text { ME }\end{array}$ & $\begin{array}{r}\text { BM } \\
\text { BC }\end{array}$ & $\begin{array}{l}408.0 \\
372.4\end{array}$ & $\overline{2} . \overline{7}$ & $\underset{ }{\mathrm{U}-\mathrm{Pb}}$ & $\begin{array}{l}\text { Zircon } \\
\text { Biotite }\end{array}$ & ${ }^{207} \mathrm{~Pb} /{ }^{206} \mathrm{~Pb}$ age; 1 concordant fraction. & This report. \\
\hline Berry Brook gabbro- & ME & BB & 410.0 & -- & ${ }^{40} \mathrm{Ar} /{ }^{39} \mathrm{Ar}$ & Hornblende & - - - & $\begin{array}{l}\text { Ludman and Idleman } \\
\text { (1998). }\end{array}$ \\
\hline Big Island Pond pluton & ME & BI & 367.7 & 1.3 & ${ }^{40} \mathrm{Ar} /{ }^{39} \mathrm{Ar}$ & Hornblende & $\begin{array}{l}\text { Average of } 2 \text { plateau ages: } 367.7 \pm 2.1 \\
\text { and } 367.6 \pm 2.1 \mathrm{Ma} \text {. }\end{array}$ & Heizler and others (1988). \\
\hline Blinn Hill granite gneiss & ME & $\mathrm{BH}$ & 424.0 & 2 & $\mathrm{U}-\mathrm{Pb}$ & Zircon & $\begin{array}{l}\text { Mean } 207 \mathrm{~Pb} / 206 \mathrm{~Pb} \text { age; } 3 \text { concordant } \\
\text { fractions. }\end{array}$ & $\begin{array}{l}\text { Tucker and others (in } \\
\text { press). }\end{array}$ \\
\hline Cadillac pluton & ME & $\mathrm{CD}$ & 419.0 & 2 & $\mathrm{U}-\mathrm{Pb}$ & Zircon & No details given. & Seaman and others (1995). \\
\hline Center Pond pluton & $\mathrm{ME}$ & $\mathrm{CP}$ & 377.0 & 3 & $\mathrm{U} \cdot \mathrm{Pb}$ & Zircon & $\begin{array}{l}\text { Mean }{ }^{207} \mathrm{~Pb} / 206 \mathrm{~Pb} \text { age; } 2 \text { concordant } \\
\text { fractions. }\end{array}$ & This report. \\
\hline Chain of Ponds pluton & $\mathrm{ME}, \mathrm{QU}$ & $\mathrm{CH}$ & 373.3 & 2 & ${ }^{40} \mathrm{Ar} /{ }^{39} \mathrm{Ar}$ & Hornblende & $\begin{array}{l}\text { Average of } 6 \text { plateau ages: } 371.6 \text {, } \\
371.1,375.4,373.2,372.5 \text {, and } \\
375.9 \mathrm{Ma} \text { (all } \pm 2.1 \mathrm{Ma}) \text {. }\end{array}$ & Heitzler and others (1988). \\
\hline Chandler pluton & ME & $\mathrm{CA}$ & 366.5 & 3.7 & ${ }^{40} \mathrm{Ar} /{ }^{39} \mathrm{Ar}$ & Biotite & Total-gas age; almost a plateau age. & This report. \\
\hline Cochrane Lake pluton & $\mathrm{ME}$ & CL & 404.3 & 3.7 & ${ }^{40} \mathrm{Ar} /{ }^{39} \mathrm{Ar}$ & Hornblende & $\begin{array}{l}\text { Average of } 3 \text { plateau ages: } 401 \pm 4, \\
405 \pm 5 \text {, and } 407 \pm 7 \mathrm{Ma} \text {. }\end{array}$ & Hubacher and Lux (1987). \\
\hline $\begin{array}{l}\text { Deblois pluton, } \\
\text { northeastern arm }\end{array}$ & ME & $\mathrm{DE}$ & 384.0 & 5 & $\mathrm{U}-\mathrm{Pb}$ & Zircon & No details given. & $\begin{array}{l}\text { A. Ludman (written } \\
\text { commun., 1998); date by }\end{array}$ \\
\hline $\begin{array}{l}\text { Deboullie pluton, } \\
\text { granodiorite }\end{array}$ & ME & DB & 364.2 & 3.2 & ${ }^{40} \mathrm{Ar} /{ }^{39} \mathrm{Ar}$ & Biotite & Plateau age. & $\begin{array}{l}\text { T. Lanzirott1. } \\
\text { This report. }\end{array}$ \\
\hline $\begin{array}{l}\text { Deboullie pluton, } \\
\text { monzonite }\end{array}$ & ME & DB & 363.9 & -- & ${ }^{40} \mathrm{Ar} /{ }^{\beta 9} \mathrm{Ar}$ & Biotite & $\begin{array}{l}\text { Average of two plateau ages: } 362.3 \pm 3 \\
\text { and } 365.4 \pm 3.2 \mathrm{Ma} \text {. }\end{array}$ & Do. \\
\hline Ebeemee pluton & ME & $\mathrm{EB}$ & 407.8 & 2.4 & ${ }^{40} \mathrm{Ar} /{ }^{39} \mathrm{Ar}$ & Biotite & Plateau age. & Do. \\
\hline Ebeemee pluton & ME & EB & 405.7 & 2.6 & $\mathrm{U}-\mathrm{Pb}$ & Zircon & $\begin{array}{l}\text { Mean }{ }^{207} \mathrm{~Pb} / 206 \mathrm{~Pb} \text { age; } 2 \text { concordant } \\
\text { zircon fractions. }\end{array}$ & Do. \\
\hline Hammond Ridge sill & ME & $\mathrm{HM}$ & 377.2 & 2.5 & $\mathrm{U}-\mathrm{Pb}$ & Zircon & $\begin{array}{l}\text { Mean } 207 \mathrm{~Pb} / 206 \mathrm{~Pb} \text { age; } 6 \text { concordant } \\
\text { fractions. }\end{array}$ & Do. \\
\hline $\begin{array}{l}\text { Harkshaw pluton, } \\
\text { Pokiok batholith }\end{array}$ & NB & HK & 411.0 & 1 & $\mathrm{U}-\mathrm{Pb}$ & Sphene & ${ }^{207} \mathrm{~Pb} / 206 \mathrm{~Pb}$ age; 1 concordant fraction. & $\begin{array}{l}\text { Bevier and Whalen } \\
(1990 \mathrm{a}) \text {. }\end{array}$ \\
\hline Harrington pluton & ME & $\mathrm{HA}$ & 406.9 & 1 & $\mathrm{U}-\mathrm{Pb}$ & Zircon & $\begin{array}{l}\text { Mean }{ }^{207} \mathrm{~Pb} / 206 \mathrm{~Pb} \text { age; } 3 \text { concordant } \\
\text { zircon fractions. }\end{array}$ & This report. \\
\hline $\begin{array}{l}\text { Hartfield pluton, } \\
\text { Pokiok batholith }\end{array}$ & NB & HT & 415.0 & 1 & $\mathrm{U}-\mathrm{Pb}$ & Sphene & ${ }^{207} \mathrm{~Pb} / 206 \mathrm{~Pb}$ age; 1 concordant fraction. & $\begin{array}{l}\text { Bevier and Whalen } \\
(1990 \mathrm{a}) \text {. }\end{array}$ \\
\hline Hartland pluton & ME & HD & 378.9 & 1.3 & $\mathrm{U}-\mathrm{Pb}$ & $\begin{array}{l}\text { Zircon and } \\
\text { sphene }\end{array}$ & $\begin{array}{l}\text { Mean }{ }^{207} \mathrm{~Pb} / 206 \mathrm{~Pb} \text { age; } 4 \text { concordant } \\
\text { fractions. }\end{array}$ & This report. \\
\hline Haskell Hill pluton & ME & HH & 408.0 & $+5 /-4$ & $\mathrm{U}-\mathrm{Pb}$ & Zircon & $\begin{array}{l}\text { Mean }{ }^{207} \mathrm{~Pb} /{ }^{206} \mathrm{~Pb} \text { age; } 1 \text { concordant } \\
\text { fraction. }\end{array}$ & $\begin{array}{l}\text { Tucker and others (in } \\
\text { press). }\end{array}$ \\
\hline $\begin{array}{l}\text { Hog Island pluton } \\
\text { Horserace quartz diorite }\end{array}$ & $\begin{array}{l}\text { ME } \\
\text { ME }\end{array}$ & $\begin{array}{l}\mathrm{HI} \\
\mathrm{HR}\end{array}$ & $\begin{array}{l}370.0 \\
392.0\end{array}$ & -- & $\begin{array}{l}\text { U-Pb } \\
\text { U.Pb }\end{array}$ & $\begin{array}{l}\text { Zircon } \\
\text { Zircon }\end{array}$ & $\begin{array}{l}{ }^{207} \mathrm{~Pb} / 206 \mathrm{~Pb} \text { age; } 1 \text { concordant fraction. } \\
207 \mathrm{~Pb} / 206 \mathrm{~Pb} \text { age; } 1 \text { concordant fraction. }\end{array}$ & $\begin{array}{l}\text { This report. } \\
\text { Tucker (unpub data) }\end{array}$ \\
\hline Hunt Ridge pluton & ME & HU & 401.5 & $\overline{4}$ & ${ }^{40} \mathrm{Ar} /{ }^{39} \mathrm{Ar}$ & $\begin{array}{l}\text { Clinopyrox- } \\
\text { ene and } \\
\text { hornblende }\end{array}$ & $\begin{array}{l}\text { Average of } 2 \text { plateau ages: } 401 \pm 4 \text { and } \\
402 \pm 5 \mathrm{Ma} \text {. }\end{array}$ & Hubacher and Lux (1987). \\
\hline Katahdin pluton & ME & $\mathrm{KA}$ & 407.0 & .4 & $\mathrm{U}-\mathrm{Pb}$ & Zircon & $\begin{array}{l}\text { Mean }{ }^{207} \mathrm{~Pb} / 206 \mathrm{~Pb} \text { age; } 5 \text { concordant } \\
\text { zircon fractions. }\end{array}$ & Rankin and Tucker (1995). \\
\hline $\begin{array}{l}\text { Lac aux Araignées } \\
\text { (Spider Lake) pluton }\end{array}$ & QU & LA & 383.0 & 3 & $\mathrm{U} \cdot \mathrm{Pb}$ & Zircon & ${ }^{207} \mathrm{~Pb} / 206 \mathrm{~Pb}$ age $; 1$ concordant fraction. & Simonetti and Doig (1990). \\
\hline $\begin{array}{l}\text { Lake Saint George } \\
\text { granite gneiss }\end{array}$ & ME & $\mathbf{L S}$ & 422.0 & 2 & $\mathrm{U}-\mathrm{Pb}$ & Zircon & $\begin{array}{l}\text { Mean }{ }^{207} \mathrm{~Pb} / 206 \mathrm{~Pb} \text { age; } 3 \text { concordant } \\
\text { fractions. }\end{array}$ & $\begin{array}{l}\text { Tucker and others (in } \\
\text { press). }\end{array}$ \\
\hline Lexington pluton & ME & $\mathrm{LX}$ & 404.2 & 1.8 & $\mathrm{U}-\mathrm{Pb}$ & Zircon & $\begin{array}{l}\text { Mean }{ }^{207} \mathrm{~Pb} / 206 \mathrm{~Pb} \text { age; } 1 \text { concordant } \\
\text { fraction. }\end{array}$ & Solar and others (1998). \\
\hline Lincoln shonkinite & ME & LI & 417.7 & 1 & $\mathrm{U}-\mathrm{Pb}$ & Zircon & $\begin{array}{l}\text { Mean } 207 \mathrm{~Pb} / 206 \mathrm{~Pb} \text { age; } 4 \text { concordant } \\
\text { fractions. }\end{array}$ & $\begin{array}{l}\text { Tucker and others (in } \\
\text { press). }\end{array}$ \\
\hline Lucerne pluton & ME & LU & 380.0 & 4 & $\mathrm{U}-\mathrm{Pb}$ & Zircon & No details given. & $\begin{array}{l}\text { Zartman and Gallego } \\
(1979) .\end{array}$ \\
\hline Magaguadavic pluton & NB & MG & 396.0 & 1 & $\mathrm{U}-\mathrm{Pb}$ & Zircon & No details given. & $\begin{array}{l}\text { M.L. Bevier (written } \\
\text { commun., 1996). }\end{array}$ \\
\hline Mattamiscontis pluton & ME & MA & 406.9 & 3.6 & $\mathrm{U}-\mathrm{Pb}$ & Zircon & $\begin{array}{l}\text { Mean }{ }^{207} \mathrm{~Pb} / 206 \mathrm{~Pb} \text { age; } 2 \text { concordant } \\
\text { zircon fractions. }\end{array}$ & This report. \\
\hline $\begin{array}{l}\text { Mixer Pond granite } \\
\text { gneiss }\end{array}$ & ME & MP & 400.0 & 3 & $\mathrm{U}-\mathrm{Pb}$ & Zircon & $\begin{array}{l}\text { Mean }{ }^{207} \mathrm{~Pb} / 205 \mathrm{~Pb} \text { age; } 2 \text { concordant } \\
\text { fractions. }\end{array}$ & $\begin{array}{l}\text { Tucker and others (in } \\
\text { press). }\end{array}$ \\
\hline $\begin{array}{l}\text { Mooselook-Meguntic } \\
\text { two-mica leucogranite }\end{array}$ & ME & MO & 370.3 & 1.1 & $\mathrm{U}-\mathrm{Pb}$ & Monazite & $\begin{array}{l}\text { Mean }{ }^{207} \mathrm{~Pb} / 206 \mathrm{~Pb} \text { age } ; 2 \text { concordant } \\
\text { fractions. }\end{array}$ & Solar and others (1998). \\
\hline $\begin{array}{l}\text { Mooselook-Meguntic } \\
\text { pluton, biotite granite }\end{array}$ & ME & MO & 388.9 & 1.6 & $\mathrm{U}-\mathrm{Pb}$ & Zircon & $\begin{array}{l}\text { Mean }{ }^{207} \mathrm{~Pb} / 206 \mathrm{~Pb} \text { age; } 3 \text { concordant } \\
\text { fractions. }\end{array}$ & Do. \\
\hline Mount Waldo pluton & ME & MW & 371.0 & 1.9 & $\mathrm{U}-\mathrm{Pb}$ & Zircon & $\begin{array}{l}\text { Mean }{ }^{207} \mathrm{~Pb} / 206 \mathrm{~Pb} \text { age; } 4 \text { concordant } \\
\text { fractions. }\end{array}$ & Stewart and others (1995b). \\
\hline $\begin{array}{l}\text { Moxie pluton, central } \\
\text { part }\end{array}$ & ME & MX & 404.4 & 3.4 & ${ }^{40} \mathrm{Ar} /{ }^{39} \mathrm{Ar}$ & Biotite & Plateau age. & This report. \\
\hline $\begin{array}{l}\text { Moxie pluton, eastern } \\
\text { part }\end{array}$ & ME & MX & 406.3 & 3.8 & ${ }^{40} \mathrm{Ar} /{ }^{39} \mathrm{Ar}$ & Biotite & Plateau age. & Do. \\
\hline Mount Douglas pluton & NB & MD & 366.5 & 1 & $\mathrm{U}-\mathrm{Pb}$ & Monazite & Mean of two U-Pb ages. & $\begin{array}{l}\text { M.L. Bevier (written } \\
\text { commun., 1996). }\end{array}$ \\
\hline $\begin{array}{l}\text { North Roxbury two- } \\
\text { mica granite }\end{array}$ & ME & NR & 404.3 & 1.9 & $\mathrm{U}-\mathrm{Pb}$ & $\begin{array}{l}\text { Zircon and } \\
\text { monazite }\end{array}$ & $\begin{array}{l}\text { Mean } 207 \mathrm{~Pb} / 206 \mathrm{~Pb} \text { age; } 3 \text { concordant } \\
\text { fractions. }\end{array}$ & Solar and others (1998). \\
\hline $\begin{array}{l}\text { North Searsmont granite } \\
\text { gneiss }\end{array}$ & $\mathrm{ME}$ & NS & 389.0 & 2 & $\mathrm{U}-\mathrm{Pb}$ & Zircon & $\begin{array}{l}\text { Mean }{ }^{207} \mathrm{~Pb} / 206 \mathrm{~Pb} \text { age; } 4 \text { concordant } \\
\text { fractions. }\end{array}$ & $\begin{array}{l}\text { Tucker and others (in } \\
\text { press). }\end{array}$ \\
\hline $\begin{array}{l}\text { North Union granitic } \\
\text { gneiss }\end{array}$ & $\mathrm{ME}$ & NU & 422.0 & 2 & $\mathrm{U}-\mathrm{Pb}$ & Zircon & $\begin{array}{l}\text { Mean }{ }^{207} \mathrm{~Pb} / 206 \mathrm{~Pb} ; 2 \text { concordant zircon } \\
\text { fractions. }\end{array}$ & Do. \\
\hline
\end{tabular}




\begin{tabular}{|c|c|c|c|c|c|c|c|c|}
\hline Pluton & $\begin{array}{l}\text { State or } \\
\text { province }\end{array}$ & $\begin{array}{l}\text { Abbrev. } \\
\text { (fig. 7) }\end{array}$ & $\begin{array}{l}\text { Age } \\
\text { (Ma) }\end{array}$ & $\begin{array}{l}\text { Error } \\
\text { (Ma) }\end{array}$ & Method & Mineral & Comments & Reference \\
\hline Northport pluton & ME & NP & 419.0 & 2 & $\mathrm{U}-\mathrm{Pb}$ & Zircon & No details given & $\begin{array}{l}\text { D.B. Stewart (written } \\
\text { commun., 1996); date by } \\
\text { R.D. Tucker. }\end{array}$ \\
\hline Onawa pluton, gabbro & $\mathrm{ME}$ & ON & 401.1 & 3.7 & ${ }^{40} \mathrm{Ar} /{ }^{39} \mathrm{Ar}$ & Biotite & Plateau age. & This report. \\
\hline $\begin{array}{l}\text { Onawa pluton, grano- } \\
\text { diorite }\end{array}$ & ME & ON & 405.1 & 2.9 & $\mathrm{U}-\mathrm{Pb}$ & Zircon & $\begin{array}{l}\text { Mean } 207 \mathrm{~Pb} / 206 \mathrm{~Pb} \text { age; } 3 \text { concordant } \\
\text { zircon fractions. }\end{array}$ & Do. \\
\hline Pegmatite, Gardiner & ME & GA & 367.0 & 1 & $\mathrm{U}-\mathrm{Pb}$ & Zircon & $\begin{array}{l}\text { Mean }{ }^{207} \mathrm{~Pb} / 206 \mathrm{~Pb} \text { age; } 3 \text { concordant } \\
\text { fractions. }\end{array}$ & $\begin{array}{l}\text { Tucker and others (in } \\
\text { press). }\end{array}$ \\
\hline $\begin{array}{l}\text { Pegmatite, Greeley } \\
\text { Corner }\end{array}$ & ME & GR & 371.0 & 1 & $\mathrm{U}-\mathrm{Pb}$ & Zircon & $\begin{array}{l}\text { Mean }{ }^{207} \mathrm{~Pb} / 206 \mathrm{~Pb} \text { age; } 2 \text { concordant } \\
\text { fractions. }\end{array}$ & Do. \\
\hline Phillips pluton & ME & PH & 403.6 & 2.2 & $\mathrm{U}-\mathrm{Pb}$ & $\begin{array}{l}\text { Zircon and } \\
\text { monazite }\end{array}$ & $\begin{array}{l}\text { Mean } 207 \mathrm{~Pb} / 206 \mathrm{~Pb} \text { age; } 3 \text { concordant } \\
\text { fractions. }\end{array}$ & Solar and others (1998). \\
\hline $\begin{array}{l}\text { Phillips pluton } \\
\text { schlieritic granite }\end{array}$ & ME & PH & 405.3 & 1.8 & $\mathrm{U}-\mathrm{Pb}$ & Zircon & $\begin{array}{l}\text { Mean }{ }^{207} \mathrm{~Pb} / 206 \mathrm{~Pb} \text { age; } 1 \text { concordant } \\
\text { fraction. }\end{array}$ & Do. \\
\hline $\begin{array}{l}\text { Phillips pluton, leuco- } \\
\text { granite }\end{array}$ & ME & PH & 403.5 & 1.6 & $\mathrm{U}-\mathrm{Pb}$ & $\begin{array}{l}\text { Zircon and } \\
\text { monazite }\end{array}$ & $\begin{array}{l}\text { Mean }{ }^{207} \mathrm{~Pb} /{ }^{206} \mathrm{~Pb} \text { age; } 3 \text { concordant } \\
\text { fractions. }\end{array}$ & Do. \\
\hline Pleasant Lake pluton & ME & PL & 401.3 & 4.7 & ${ }^{40} \mathrm{Ar} /{ }^{39} \mathrm{Ar}$ & Biotite & $\begin{array}{l}\text { Average of } 3 \text { plateau ages: } 404 \pm 5 \text {, } \\
397 \pm 9 \text {, and } 403 \pm 7 \mathrm{Ma} \text {. }\end{array}$ & Hubacher and Lux (1987). \\
\hline Pleasant Lake pluton & ME & PL & 400.5 & 4.5 & ${ }^{40} \mathrm{Ar} /{ }^{39} \mathrm{Ar}$ & Hornblende & $\begin{array}{l}\text { Average of } 2 \text { plateau ages: } 400 \pm 5 \text { and } \\
401 \pm 4 \mathrm{Ma} \text {. }\end{array}$ & Do. \\
\hline $\begin{array}{l}\text { Pocomoonshine gabbro- } \\
\text { diorite }\end{array}$ & ME & PO & 422.7 & 3.0 & ${ }^{40} \mathrm{Ar} /{ }^{39} \mathrm{Ar}$ & Hornblende & $\begin{array}{l}\text { Average of two plateau ages: } 423.5 \pm 2.7 \\
\text { and } 421.5 \pm 3.0 \mathrm{Ma} \text {. Dates intrusion of } \\
\text { more northerly mafic phase. }\end{array}$ & West and others (1992). \\
\hline Priestly pluton & ME & PR & 361.7 & -- & ${ }^{40} \mathrm{Ar} /{ }^{39} \mathrm{Ar}$ & Biotite & $\begin{array}{l}\text { Average of two plateau ages: } \\
360.3 \pm 2.78 \text { and } 363.2 \pm 2.3 \mathrm{Ma} \text {. }\end{array}$ & This report. \\
\hline Priestly pluton & $\mathrm{ME}$ & PR & 361.0 & 3 & $\mathrm{U}-\mathrm{Pb}$ & Zircon & ${ }^{207} \mathrm{~Pb} / 206 \mathrm{~Pb}$ age; 1 concordant fraction. & Do. \\
\hline $\begin{array}{l}\text { Redington biotite } \\
\text { granite }\end{array}$ & ME & RD & 407.6 & 4.7 & $\mathrm{U}-\mathrm{Pb}$ & Zircon & $\begin{array}{l}\text { Mean }{ }^{207} \mathrm{~Pb} / 206 \mathrm{~Pb} \text { age; } 3 \text { concordant } \\
\text { fractions. }\end{array}$ & Solar and others (1998). \\
\hline Rome pluton & ME & RO & 378.0 & 1 & $\mathrm{U}-\mathrm{Pb}$ & Zircon & $\begin{array}{l}\text { Mean } 207 \mathrm{~Pb} / 206 \mathrm{~Pb} \text { age; } 3 \text { concordant } \\
\text { fractions. }\end{array}$ & $\begin{array}{l}\text { Tucker and others (in } \\
\text { press). }\end{array}$ \\
\hline $\begin{array}{l}\text { Russell Mountain } \\
\text { pluton }\end{array}$ & ME & RU & 406.0 & 1.3 & $\mathrm{U}-\mathrm{Pb}$ & Zircon & No details given. & This report. \\
\hline Scotstown pluton & QU & $\mathrm{SC}$ & 384.0 & 2 & $\mathrm{U} \cdot \mathrm{Pb}$ & Zircon & $\begin{array}{l}\text { Mean }{ }^{207} \mathrm{~Pb} /{ }^{206} \mathrm{~Pb} \text { age of } 2 \text { slightly } \\
\text { discordant fractions. }\end{array}$ & Simonetti and Doig (1990). \\
\hline Sebec Lake pluton & ME & SL & 407.8 & 2.5 & $\mathrm{U}-\mathrm{Pb}$ & Zircon & $\begin{array}{l}\text { Mean } 207 \mathrm{~Pb} / 206 \mathrm{~Pb} \text { age; } 2 \text { concordant } \\
\text { zircon fractions. }\end{array}$ & This report. \\
\hline $\begin{array}{l}\text { Seboeis granodiorite at } \\
\text { East Branch Lake }\end{array}$ & ME & SE & 364.7 & 4.3 & ${ }^{40} \mathrm{Ar} /{ }^{39} \mathrm{Ar}$ & Biotite & Plateau age. & Do. \\
\hline $\begin{array}{l}\text { Seboeis pluton, } \\
\text { granodiorite }\end{array}$ & ME & SS & 405.8 & 2.5 & $\mathrm{U} \cdot \mathrm{Pb}$ & Zircon & $\begin{array}{l}\text { Mean }{ }^{207} \mathrm{~Pb} / 206 \mathrm{~Pb} ; 1 \text { concordant zircon } \\
\text { fraction. }\end{array}$ & Do. \\
\hline Sedgwick pluton & ME & SG & 419.5 & 1.4 & $\mathrm{U}-\mathrm{Pb}$ & Zircon & $\begin{array}{l}\text { Mean }{ }^{207} \mathrm{~Pb} / 206 \mathrm{~Pb} \text { age; } 3 \text { concordant } \\
\text { fractions. }\end{array}$ & Stewart and others (1995b). \\
\hline $\begin{array}{l}\text { Shirley-Blanchard } \\
\text { composite pluton }\end{array}$ & ME & SB & 406.9 & 1.4 & $\mathrm{U}-\mathrm{Pb}$ & Zircon & $\begin{array}{l}\text { Mean } 207 \mathrm{~Pb} / 206 \mathrm{~Pb} ; 4 \text { concordant zircon } \\
\text { fractions. }\end{array}$ & This report. \\
\hline $\begin{array}{l}\text { Skiff Lake pluton, } \\
\text { Pokiok batholith }\end{array}$ & NB & SK & 409.0 & 2 & $\mathrm{U}-\mathrm{Pb}$ & Zircon & $\begin{array}{l}207 \mathrm{~Pb} / 206 \mathrm{~Pb} \text { age of most concordant } \\
\text { fraction. }\end{array}$ & $\begin{array}{l}\text { Bevier and Whalen } \\
(1990 a) \text {. }\end{array}$ \\
\hline Somesville pluton & ME & $\begin{array}{l}\text { SV } \\
\text { SO }\end{array}$ & $\begin{array}{l}424.0 \\
382.0\end{array}$ & 2 & $\mathrm{U}-\mathrm{Pb}$ & Zircon & No details given. & Seaman and others (1995). \\
\hline Songo pluton & ME & SO & 382.0 & 3 & $\mathrm{U}-\mathrm{Pb}$ & $\begin{array}{l}\text { Zircon and } \\
\text { sphene }\end{array}$ & $\begin{array}{l}\text { Mean }{ }^{207} \mathrm{~Pb} / 206 \mathrm{~Pb} \text { age; } 2 \text { slightly } \\
\text { discordant fractions. }\end{array}$ & $\begin{array}{l}\text { Lux and Aleinikoff (1985), } \\
\text { Lux and others (1989). }\end{array}$ \\
\hline South Penobscot pluton & ME & $\mathrm{SP}$ & 419.2 & 2.2 & $\mathrm{U}-\mathrm{Pb}$ & Zircon & $\begin{array}{l}\text { Supersedes date of Stewart and others } \\
\text { (1995). }\end{array}$ & $\begin{array}{l}\text { D.B. Stewart (written } \\
\text { commun., 1996); date by } \\
\text { R.D. Tucker. }\end{array}$ \\
\hline $\begin{array}{l}\text { South Roxbury two- } \\
\text { mica granite }\end{array}$ & ME & SR & 408.2 & 2.5 & $\mathrm{U}-\mathrm{Pb}$ & $\begin{array}{l}\text { Zircon and } \\
\text { monazite }\end{array}$ & $\begin{array}{l}\text { Mean }{ }^{207} \mathrm{~Pb} / 206 \mathrm{~Pb} \text { age; } 4 \text { concordant } \\
\text { fractions. }\end{array}$ & Solar and others (1998). \\
\hline Spruce Head granite & ME & SH & 421.0 & 1 & $\mathrm{U}-\mathrm{Pb}$ & $\begin{array}{l}\text { Zircon and } \\
\text { sphene }\end{array}$ & $\begin{array}{l}\text { Mean }{ }^{207} \mathrm{~Pb} / 206 \mathrm{~Pb} \text { age; } 4 \text { concordant } \\
\text { fractions. }\end{array}$ & $\begin{array}{l}\text { Tucker and others (in } \\
\text { press). }\end{array}$ \\
\hline Ste-Cécile pluton & QU & ST & 374.0 & 1 & $\mathrm{U}-\mathrm{Pb}$ & Zircon & $\begin{array}{l}\text { Mean }{ }^{207} \mathrm{~Pb} / 206 \mathrm{~Pb} \text { age; } 3 \text { concordant or } \\
\text { nearly concordant fractions. }\end{array}$ & Simonetti and Doig (1990). \\
\hline $\begin{array}{l}\text { Swift River two-mica } \\
\text { leucogranite }\end{array}$ & ME & SW & 407.9 & 1.9 & $\mathrm{U}-\mathrm{Pb}$ & Zircon & $\begin{array}{l}\text { Mean }{ }^{207} \mathrm{~Pb} / 206 \mathrm{~Pb} \text { age; } 3 \text { concordant } \\
\text { fractions. }\end{array}$ & Solar and others (1998). \\
\hline $\begin{array}{l}\text { Thrasher Peaks } \\
\text { intrusions }\end{array}$ & $\mathrm{ME}$ & TH & 414.0 & -- & $\mathrm{U}-\mathrm{Pb}$ & Zircon & ${ }^{207} \mathrm{~Pb} / 206 \mathrm{~Pb}$ age $; 1$ concordant fraction. & Moench and others (1995). \\
\hline Threemile Pond pluton & ME & TP & 381.0 & 1 & $\mathrm{U}-\mathrm{Pb}$ & $\begin{array}{l}\text { Zircon and } \\
\text { sphene }\end{array}$ & $\begin{array}{l}\text { Mean }{ }_{\text {fractions. }}^{207} \mathrm{~Pb} / 206 \mathrm{~Pb} \text { age; } 4 \text { concordant } \\
\text {. }\end{array}$ & $\begin{array}{l}\text { Tucker and others (in } \\
\text { press). }\end{array}$ \\
\hline Togus pluton & $\mathrm{ME}$ & TO & 378.0 & 1 & $\mathrm{U}-\mathrm{Pb}$ & Zircon & $\begin{array}{l}\text { Mean }{ }^{207} \mathrm{~Pb} / 206 \mathrm{~Pb} \text { age; } 2 \text { concordant } \\
\text { fractions. }\end{array}$ & Do. \\
\hline Umbagog pluton & ME & UM & 384.0 & 6 & $\mathrm{U}-\mathrm{Pb}$ & Zircon & No details given. & $\begin{array}{l}\text { Aleinikoff and Moench } \\
(1987) .\end{array}$ \\
\hline Utopia pluton & NB & UT & 422.7 & 1 & $\mathrm{U}-\mathrm{Pb}$ & Zircon & No details given. & $\begin{array}{l}\text { M.L. Bevier (written } \\
\text { commun., 1996). }\end{array}$ \\
\hline $\begin{array}{l}\text { Waldoboro binary } \\
\text { granite }\end{array}$ & ME & WD & 367.8 & 1.6 & $\mathrm{U}-\mathrm{Pb}$ & Zircon & $\begin{array}{l}\text { Mean }{ }^{207} \mathrm{~Pb} / 206 \mathrm{~Pb} \text { age; } 3 \text { concordant } \\
\text { fractions. }\end{array}$ & $\begin{array}{l}\text { Tucker and others (in } \\
\text { press). }\end{array}$ \\
\hline Wamsutta pluton & NH & WM & 408.0 & 1.9 & $\mathrm{U}-\mathrm{Pb}$ & Zircon & No details given. & $\begin{array}{l}\text { J.D. Eusden, Jr. (written } \\
\text { commun., 1998). }\end{array}$ \\
\hline Waterville granitic dike & ME & WA & 399.4 & .7 & U-Pb & Zircon & $\begin{array}{l}\text { Mean }{ }^{207} \mathrm{~Pb} / 206 \mathrm{~Pb} \text { age; } 2 \text { concordant } \\
\text { fractions. Intrudes first-generation } \\
\text { Acadian folds but is involved in } \\
\text { second-generation folds. }\end{array}$ & $\begin{array}{l}\text { Tucker and others (in } \\
\text { press). }\end{array}$ \\
\hline Welsford pluton & NB & WL & 422.0 & 1 & $\mathrm{U}-\mathrm{Pb}$ & Zircon & No details given. & $\begin{array}{l}\text { M.L. Bevier (written } \\
\text { commun., 1996). }\end{array}$ \\
\hline Winslow pluton & QU & WI & 377.0 & 6 & U.Pb & Sphene & $\begin{array}{l}\text { Mean }{ }^{207} \mathrm{~Pb} / 206 \mathrm{~Pb} \text { age; } 2 \text { nearly } \\
\text { concordant fractions. }\end{array}$ & Simonetti and Doig (1990). \\
\hline Youngtown pluton & ME & YT & 420.0 & 2 & $\mathrm{U}-\mathrm{Pb}$ & Zircon & $\begin{array}{l}\text { Mean } 207 \mathrm{~Pb} / 206 \mathrm{~Pb} \text { age; } 3 \text { concordant } \\
\text { fractions. }\end{array}$ & $\begin{array}{l}\text { Tucker and others (in } \\
\text { press). }\end{array}$ \\
\hline
\end{tabular}


Table 4. U-Pb isotope-dilution analyses of zircon from plutonic rocks, Maine-Continued.

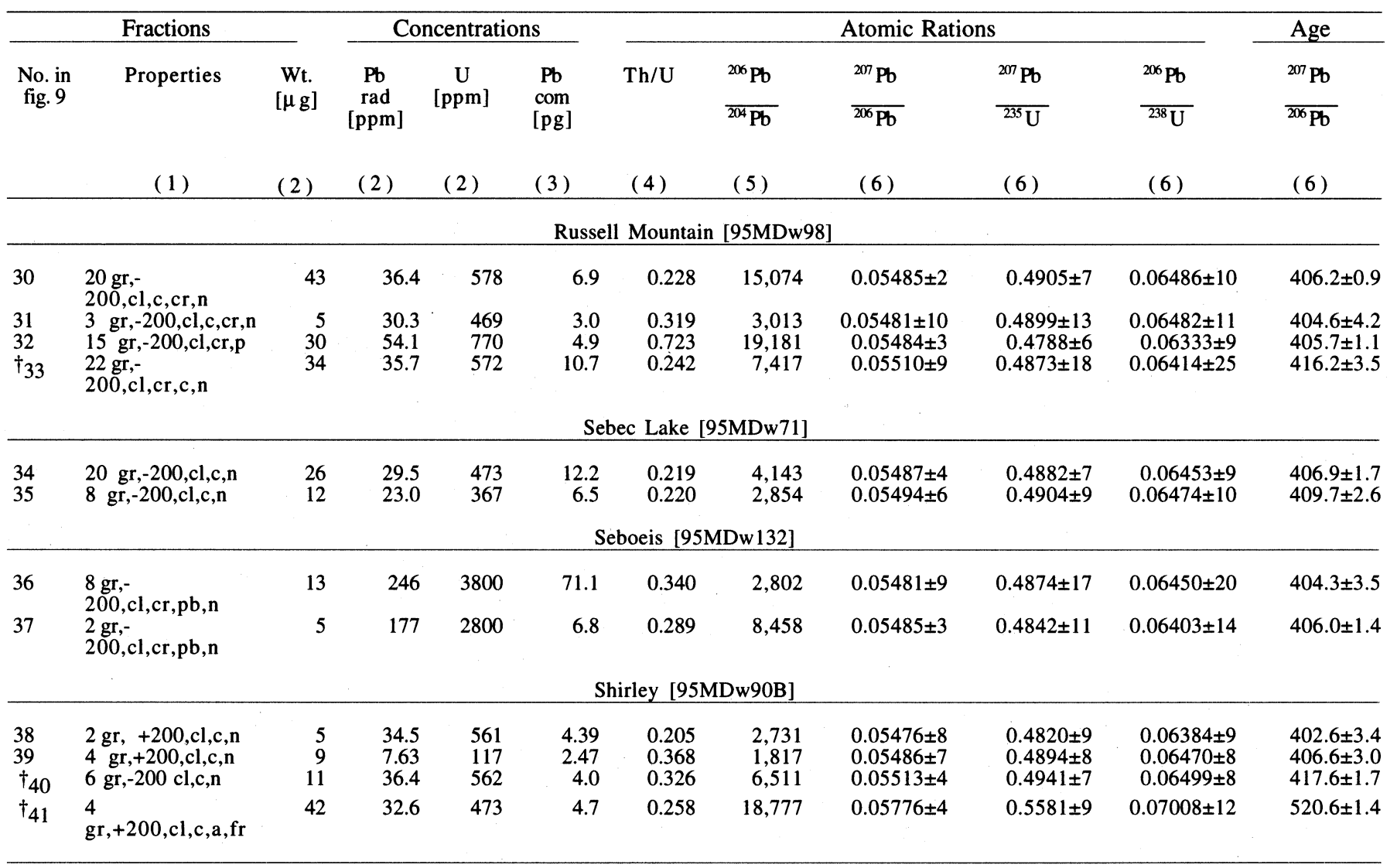

Notes:

(1) Cardinal number indicates the number of zircon grains analyzed (e.g., 35 grains); all grains were selected from nonparamagnetic separates at 0 degree tilt at full magnetic field in Frantz Magnetic Separator; $+200=$ size in mesh $(>75 \mu \mathrm{m}) ; \mathrm{c}=$ colorless; cl= clear; el $=$ elongate; $\mathrm{e}=$ equant; $\mathrm{eu}=$ euhedral; $\mathrm{f}=$ faceted; $\mathrm{l}=$ long prismatic; $\mathrm{n}=5: 1$ prismatic needles; $\mathrm{p}=$ prismatic; $\mathrm{pb}=$ pale brown; $\mathrm{s}=$ stubby; $\mathrm{s}-\mathrm{p}=$ short-prismatic; $\mathrm{t}=$ tips from prisms. $\mathrm{NA}=$ nonabraded fraction; all other grains were air-abraded following Krogh (1982). $\dagger$ $=$ analyses rejected from regression analysis because of inheritance.

(2) Concentrations are known to $\pm 30 \%$ for sample weights of about $20 \mu \mathrm{g}$ and $\pm 50 \%$ for samples $<5 \mu \mathrm{g}$

(3) Corrected for 0.0215 mole fraction common- $\mathrm{Pb}$ in the ${ }^{205} \mathrm{~Pb}-{ }^{235} \mathrm{U}$ spike.

(4) Calculated $\mathrm{Th} / \mathrm{U}$ ratio assuming that all ${ }^{208} \mathrm{~Pb}$ in excess of blank, common- $\mathrm{Pb}$, and spike is radiogenic $\left(\lambda^{232} \mathrm{Th}=4.9475 \times 10^{-11} \mathrm{y}^{-1}\right)$.

(5) Measured, uncorrected ratio.

(6) Ratio corrected for fractionation, spike, blank, and initial common- $\mathrm{Pb}$ (at the determined age from Stacey and Kramers (1975)). $\mathrm{Pb}$ fractionation correction $=0.094 \% / \mathrm{amu}( \pm 0.025 \% 1 \sigma) ; \mathrm{U}$ fractionation correction $=0.111 \% / \mathrm{amu}( \pm 0.02 \% 1 \sigma)$. U blank $=0.2 \mathrm{pg} ; \mathrm{Pb}$ blank $<=10 \mathrm{pg}$. Absolute uncertainties $(1 \sigma)$ in the $\mathrm{Pb} / \mathrm{U}$ and ${ }^{207} \mathrm{~Pb} /{ }^{206} \mathrm{~Pb}$ ratios calculated following Ludwig (1980). U and $\mathrm{Pb}$ half-lives and isotopic abundance ratios from Jaffey and others (1971).

\section{Ebeemee Pluton}

The Ebeemee pluton (EB, fig. 6) is a small, unfoliated stock of biotite granite; fieldwork for this study was limited to sampling in the pluton and a fruitless search for informative outcrops in the contact aureole. Reconnaissance mapping compiled by Osberg and others (1985) showed the pluton cutting a doubly plunging anticline cored by the Madrid Formation and flanked by the Carrabassett Formation; on this basis, the pluton would appear to postdate Acadian folding. Two concordant zircon analyses yielded a ${ }^{207} \mathrm{~Pb} /{ }^{206} \mathrm{~Pb}$ age of 405.7 $\pm 2.6 \mathrm{Ma}$, and a biotite separate yielded an ${ }^{40} \mathrm{Ar} /{ }^{39} \mathrm{Ar}$ plateau age of $407.8 \pm 2.4 \mathrm{Ma}$ (fig. 10).

\section{Seboeis Pluton}

The Seboeis pluton (SS, fig. 6) is shown on the bedrock geologic map of Maine (Osberg and others, 1985) as a large ( 5 by $30 \mathrm{~km}$ ) body that cuts the Madrid and Smalls Falls Formations and intervening faults. Because the pluton has not been mapped in detail and was sampled only in reconnaissance fashion, the following observations are necessarily preliminary. We dated four phases. Weakly foliated granodiorite at Schoodic Lake yielded two concordant zircon fractions that give a mean ${ }^{207} \mathrm{~Pb} /{ }^{206} \mathrm{~Pb}$ age of $405.8 \pm 2.5 \mathrm{Ma}$ (fig. 7). Near Dudley Rips, an unfoliated granite that yielded an ${ }^{40} \mathrm{Ar} /{ }^{39} \mathrm{Ar}$ muscovite plateau age of $401.3 \pm 2.8 \mathrm{Ma}$ (fig. 8 ) intrudes a tec- 

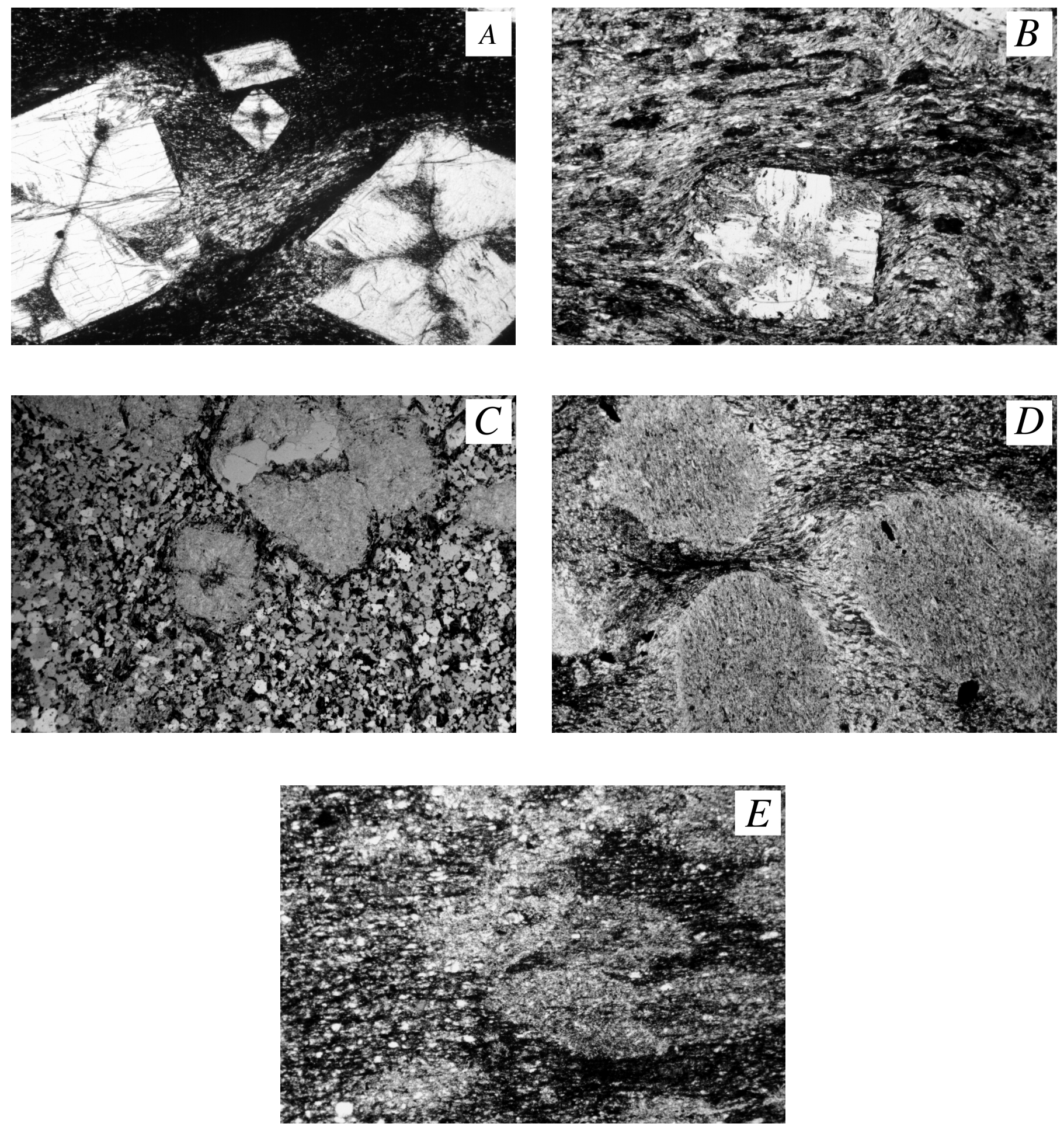

Figure 9.-Thin-section photomicrographs of contact-metamorphosed rocks adjacent to Acadian plutons. All sections are cut normal to foliation and are viewed in plane-polarized light. Field of view in each photomicrograph is about $4 \mathrm{~mm}$ across. $A$, Syntectonic metamorphic textures in aureole of the Russell Mountain pluton. Large, randomly oriented chiastolite phenocrysts are flanked by strain shadows containing aligned flakes of biotite, implying some deformation during contact metamorphism. $B$, Syntectonic metamorphic textures in aureole of the Sebec Lake pluton. Chiastolite phenocrysts are flanked by strain shadows containing aligned chlorite and biotite, implying some deformation during contact metamorphism. $C$, Static metamorphic textures in aureole of the Bald Mountain pluton, showing randomly oriented, retrograded chiastolite phenocrysts and randomly oriented biotite. Rock shows no vestige of pervasive regional foliation found outside contact aureole and so may have been metamorphosed before regional deformation. Alternatively, contact metamorphism might have been intense enough to have obliterated all preexisting fabrics. $D$, Syntectonic metamorphic textures in aureole of the Hog Island pluton. Large cordierite phenocrysts overgrow a preexisting foliation defined by aligned biotite and white mica; this first foliation now survives only within cordierite grains. A second, postcordierite foliation is defined by biotite in pressure shadows. Although metamorphic textures in this rock are broadly syntectonic, on the basis of regional relations, the first Acadian deformation probably preceded emplacement of the Hog Island pluton by 15 to 20 m.y. E, Posttectonic metamorphic textures in aureole of the Priestly pluton. Cordierite phenocrysts overgrow a weak foliation that lies at a high angle to bedding; biotite in groundmass is randomly oriented. 
tonically foliated diorite that yielded a slightly hump shaped ${ }^{40} \mathrm{Ar} /{ }^{39} \mathrm{Ar}$ age spectrum with a total-gas age of $396.3 \pm 3.8$ Ma (fig. 8). Evidently, the diorite was intruded before 401 $\mathrm{Ma}$ and before at least some of the regional deformation. A much younger biotite age from rocks that had been previously mapped as part of the Seboeis pluton at East Branch Lake is discussed below in the subsection entitled "Givetian to Early Tournaisian Plutons."

\section{Moxie Pluton}

The northwestern (inboard) part of the Emsian plutonic belt is dominated by two large intrusions: the Moxie gabbro and the Katahdin quartz monzonite. The intrusive relations of the Moxie pluton (MX, fig. 6) differ considerably from one end to the other. In the Jo Mary Mountain area at the pluton's east end (loc. 30, fig. 1), the contact aureole contains remarkably pristine, gently dipping strata that display perfectly preserved crosslaminae (Hanson and Bradley, 1989). We observed no evidence of contact-metamorphic minerals overgrowing a regional slaty cleavage, which is visible everywhere outside the aureole. Midlength along the pluton (at loc. 44, fig. 1), however, contact-metamorphic andalusite overgrows an axial-planar cleavage associated with nearly recumbent mesoscale folds. At the pluton's west end, rocks of the contact aureole show a strong foliation defined by biotite and white mica and by the preferred orientation of andalusite with asymmetric strain shadows of biotite. Thus, at three places where we have anecdotal observations, the pluton shows local evidence of predating regional Acadian folding, of postdating Acadian folding, and of synkinematic emplacement. We dated the Moxie pluton in its eastern and central sections and obtained ${ }^{40} \mathrm{Ar} /{ }^{39} \mathrm{Ar}$ biotite plateau ages of 404.4 \pm 3.4 and 406.3 $\pm 3.8 \mathrm{Ma}$, respectively (fig. 8).

\section{Katahdin Pluton}

The Katahdin pluton (KA, fig. 6) is a large (60 by $35 \mathrm{~km}$ ) body of unfoliated quartz monzonite. It has long had an enigmatic age relationship to the Acadian deformation, but this problem can now be resolved-or, at least, narrowed-with a better time scale and precise U-Pb ages (Bradley and others, 1996; Rankin and Tucker, 1995). The Katahdin pluton intrudes rocks as young as the Seboomook Group (Pragian), the Matagamon Sandstone (Pragian and earliest Emsian), and the pluton's own volcanic carapace, the Traveler Rhyolite (406 Ma; Rankin and Tucker, 1995). Near Harrington Lake (HA, fig. 6), the Seboomook Group near the pluton consists of gently dipping hornfelsed siltstone and sandstone that preserve pristine crossbedding. These rocks show no sign of ever having acquired the regional, northeast-striking, axial-planar cleavage that is pervasive just outside the contact aureole and that typically obscures or obliterates delicate sedimentary structures. In this area, the rocks in the contact aureole were baked and never cleaved, suggesting that the pluton is locally pretectonic. Along the pluton's northeastern border, in contrast, Neumann (1967) mapped a breccia zone consisting of plastically deformed metasedimentary-rock fragments, set in a gneissic granitic matrix. These rocks appear to have formed along a syntectonic intrusive contact. The Katahdin pluton resisted many early attempts to date it accurately, but Denning and Lux (1989) reported an ${ }^{40} \mathrm{Ar} /{ }^{39} \mathrm{Ar}$ biotite plateau age of 400.1 \pm 1 Ma. More recently, Rankin and Tucker (1995) reported a ${ }^{207} \mathrm{~Pb} /{ }^{206} \mathrm{~Pb}$ age of $406.9 \pm 0.4 \mathrm{Ma}$ that is the weighted average of five concordant zircon fractions.

\section{Harrington Pluton}

The Harrington pluton (HA, fig. 6) is a laccolith-like satellite to the Katahdin pluton (Griscom, 1976). Its map pattern (Griscom, 1976) implies that it may have intruded a folding sedimentary section because its basal contact is planar and concordant with homoclinally dipping beds in the subjacent Seboomook Group, whereas its upper contact is concordant with regionally folded bedding in the overlying part of the Seboomook Group. The Harrington pluton yielded three concordant zircon analyses, with a mean ${ }^{207} \mathrm{~Pb} /{ }^{206} \mathrm{~Pb}$ age of $406.9 \pm 1 \mathrm{Ma}$ (fig. 7), which suggests an Emsian age for the folding.

\section{Late Emsian to Eifelian Plutons}

A few plutons in the age range 400-387.5 Ma are scattered across the study area (fig. 6) but form no obvious pattern. The Magagauadavic pluton in southern New Brunswick has a U-Pb age of 396 \pm 1 Ma (MG, fig. 6; M.L. Bevier, written commun., 1996). A biotite granite phase of the Mooselookmeguntic pluton (MO, fig. 6) yielded a U-Pb age of 388.9 \pm 1.6 Ma (Solar and others, 1998). The North Searsmont granitic

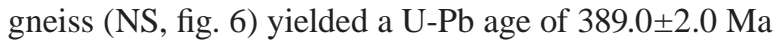
(Tucker and others, in press). The Horserace quartz diorite (HR, fig. 6), a small body within the Katahdin batholith, yielded a concordant $\mathrm{U}-\mathrm{Pb}$ zircon age of $392 \pm 3.1 \mathrm{Ma}$ (R.D. Tucker, unpub. data); an ${ }^{40} \mathrm{Ar} /{ }^{39} \mathrm{Ar}$ hornblende age of 374.9 $\pm 3.2 \mathrm{Ma}$ was previously reported for this pluton (Denning and Lux, 1989).

\section{Givetian to Early Tournasian Plutons ${ }^{2}$}

\section{Previous Results}

The best-defined group of intrusions of this age consists of Frasnian plutons in central Maine. Tucker and others (in press) recently obtained $\mathrm{U}-\mathrm{Pb}$ ages for four of these plutons: the Hartland (379 $\pm 1 \mathrm{Ma}$; HD, fig. 6), Old Point (379 $\pm 3 \mathrm{Ma}$; OP, fig. 6), Rome (378 \pm 1 Ma; RO, fig. 6), and Togus (378 \pm 1 Ma; TO, fig. 6). New ages are reported below for the Center Pond pluton and a sill at Hammond Ridge, which clearly belong in this group. Two other plutons that may belong in the same group crop out near the Maine coast: the Lucerne

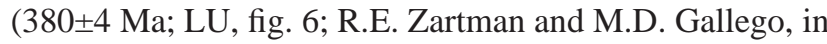
Marvin and Dobson, 1979, p. 18) and the Deblois (384 $\pm 5 \mathrm{Ma}$;

${ }^{2}$ Plutons in this age range are widespread in the area of figure 6 . In hindsight, they are not particularly relevant to the main point of this report, but they are important to late Acadian events, and their place in the scheme of things could only have been learned by obtaining reliable ages. 


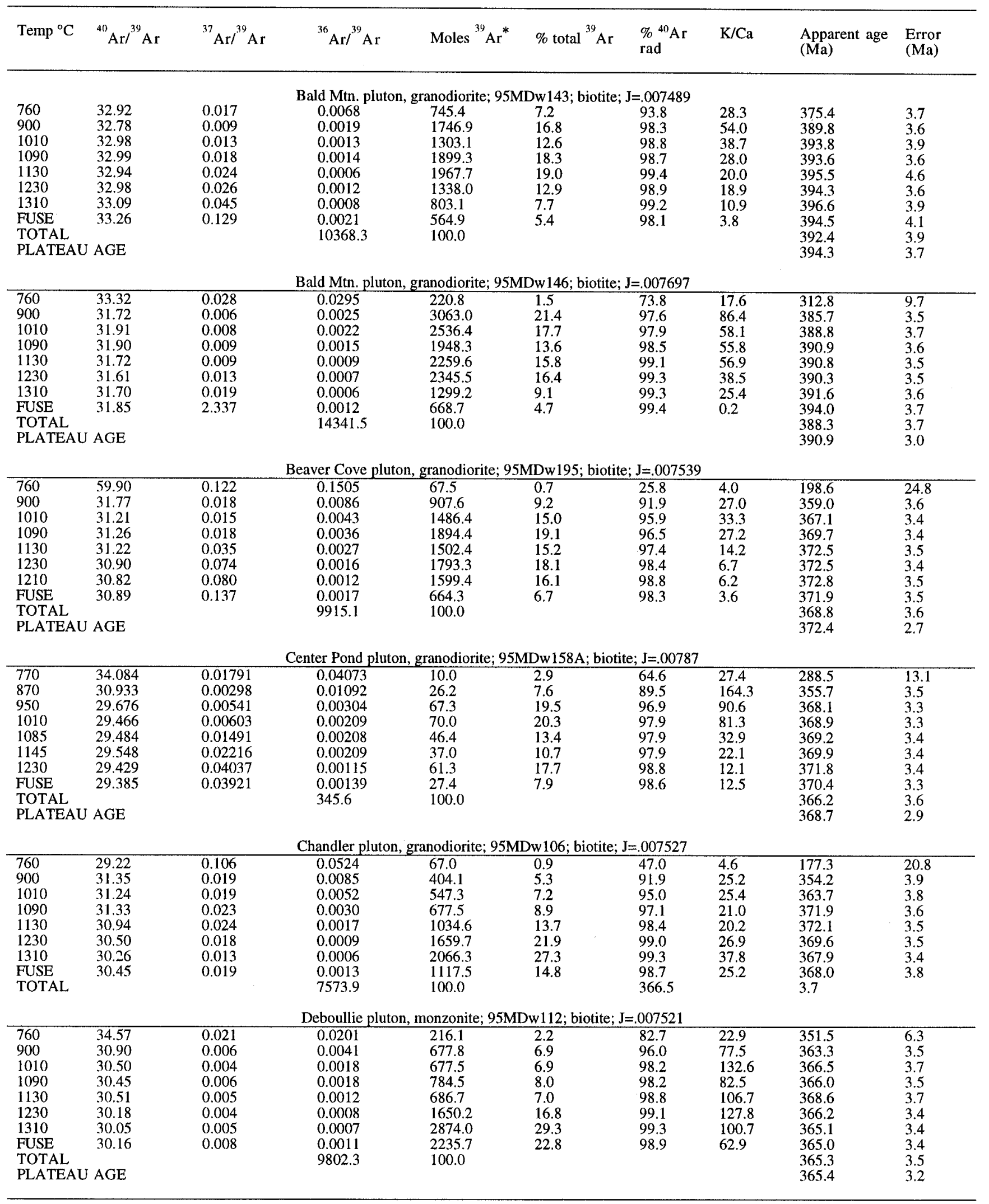


Table $5 .{ }^{40} \mathrm{Ar} /{ }^{39} \mathrm{Ar}$ data for plutonic rocks, Maine-Continued.

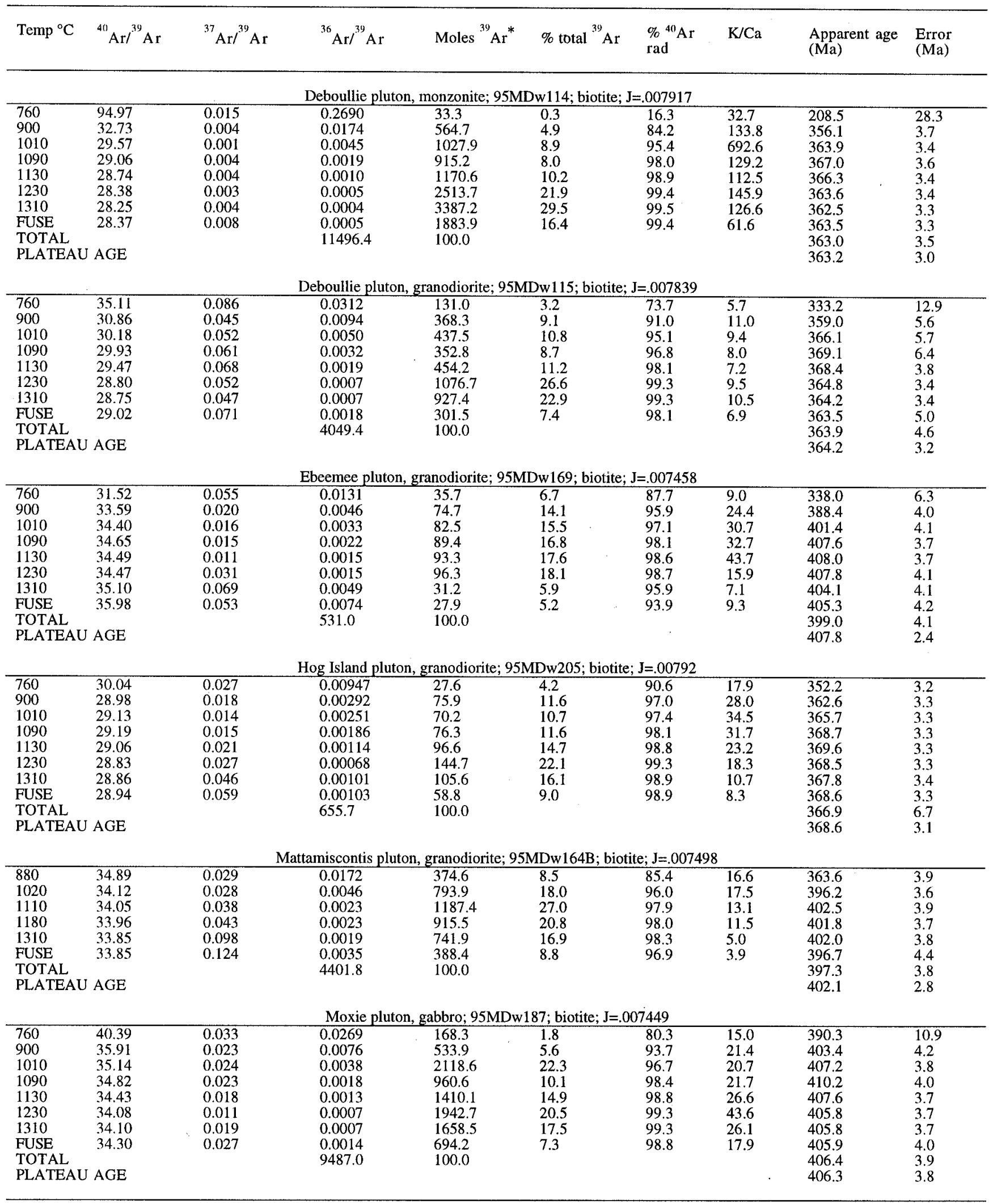




\begin{tabular}{|c|c|c|c|c|c|c|c|c|}
\hline Temp ${ }^{\circ} \mathrm{C}$ & ${ }^{40} \mathrm{Ar} /{ }^{39} \mathrm{Ar}$ & ${ }^{37} \mathrm{Arr} /{ }^{39} \mathrm{Ar}$ & ${ }^{36} \mathrm{Ar} /{ }^{39} \mathrm{Ar}$ & Moles ${ }^{39}$ Ar $^{*}$ & $\%$ total ${ }^{39} \mathrm{Ar}$ & $\%^{40} \mathrm{Ar}$ & $\mathrm{K} / \mathrm{Ca}$ & $\begin{array}{ll}\text { Apparent age } & \begin{array}{l}\text { Error } \\
\text { (Ma) }\end{array} \\
\text { (Ma) }\end{array}$ \\
\hline
\end{tabular}

\begin{tabular}{|c|c|c|c|c|c|c|c|c|c|}
\hline \multicolumn{10}{|c|}{ Moxie pluton, gabbro; 95MDw198; biotite; $J=.007962$} \\
\hline 760 & 88.47 & 0.229 & 0.23360 & 2.4 & 0.6 & 22.0 & 2.1 & 259.7 & 41.4 \\
\hline 900 & 37.51 & 0.085 & 0.02270 & 20.4 & 5.3 & 82.1 & 5.8 & 395.6 & 3.9 \\
\hline 1010 & 34.38 & 0.086 & 0.00941 & 34.6 & 9.0 & 91.9 & 5.7 & 404.8 & 3.6 \\
\hline 1090 & 33.25 & 0.083 & 0.00474 & 47.2 & 12.3 & 95.8 & 5.9 & 407.7 & 3.7 \\
\hline 1130 & 32.47 & 0.059 & 0.00284 & 67.1 & 17.4 & 97.4 & 8.4 & 405.2 & 3.6 \\
\hline 1230 & 32.00 & 0.038 & 0.00140 & 104.1 & 27.1 & 98.7 & 13.0 & 404.5 & 3.6 \\
\hline 1310 & 31.88 & 0.038 & 0.00103 & 76.4 & 19.8 & 99.0 & 12.9 & 404.5 & 3.6 \\
\hline FUSE & 32.07 & 0.048 & 0.00194 & 32.6 & $8.5^{\circ}$ & 98.2 & 10.2 & 403.5 & 3.7 \\
\hline \multirow{2}{*}{\multicolumn{2}{|c|}{ TOTAL }} & & 384.7 & 100.0 & & & & 403.6 & 3.9 \\
\hline & $\mathrm{AGE}$ & & & & & & & 404.4 & 3.4 \\
\hline
\end{tabular}

\begin{tabular}{|c|c|c|c|c|c|c|c|c|c|}
\hline \multicolumn{10}{|c|}{ Onawa pluton, gabbro phase; 95MDw68B; biotite; $\mathrm{J}=.007633$} \\
\hline 760 & 40.59 & 0.048 & 0.0586 & 19.9 & 2.3 & 57.3 & 10.1 & 294.7 & 6.4 \\
\hline 900 & 33.78 & 0.020 & 0.0073 & 126.0 & 14.5 & 93.5 & 24.6 & 389.9 & 4.0 \\
\hline 1010 & 33.43 & 0.016 & 0.0041 & 176.3 & 20.2 & 96.4 & 31.3 & 396.6 & 3.6 \\
\hline 1090 & 33.57 & 0.020 & 0.0026 & 104.0 & 11.9 & 97.7 & 24.9 & 402.9 & 3.7 \\
\hline 1130 & 33.15 & 0.026 & 0.0014 & 144.1 & 16.5 & 98.7 & 19.0 & 402.3 & 3.7 \\
\hline 1230 & 32.69 & 0.015 & 0.0005 & 97.2 & 11.1 & 99.5 & 33.6 & 400.2 & 3.6 \\
\hline 1310 & 32.80 & 0.013 & 0.0006 & 115.2 & 13.2 & 99.4 & 37.7 & 401.0 & 3.9 \\
\hline FUSE & 32.87 & 0.038 & 0.0014 & 89.4 & 10.2 & 98.7 & 12.8 & 399.2 & 3.9 \\
\hline \multirow{2}{*}{\multicolumn{2}{|c|}{$\begin{array}{l}\text { TOTAL } \\
\text { PLATEA }\end{array}$}} & & 872.1 & 100.0 & & & & 396.2 & 3.8 \\
\hline & & & & & & & & 400.1 & 3.7 \\
\hline
\end{tabular}

PLATEAU AGE

Priestly pluton, granodiorite; 95MDw107; biotite; J=.00786

\begin{tabular}{|c|c|c|c|c|c|c|c|c|c|}
\hline 760 & 31.01 & 0.010 & 0.0159 & 356.9 & 3.4 & 84.8 & 49.2 & 338.9 & 4.5 \\
\hline 900 & 29.47 & 0.013 & 0.0061 & 756.1 & 7.2 & 93.8 & 37.7 & 354.7 & 3.5 \\
\hline 1010 & 29.33 & 0.022 & 0.0043 & 938.9 & 9.0 & 95.6 & 22.7 & 359.3 & 3.5 \\
\hline 1090 & 29.18 & 0.051 & 0.0033 & 1102.0 & 10.5 & 96.6 & 9.6 & 361.2 & 3.6 \\
\hline 1130 & 28.88 & 0.060 & 0.0020 & 1497.7 & 14.3 & 97.9 & 8.1 & 362.0 & 3.4 \\
\hline 1230 & 28.47 & 0.038 & 0.0012 & 2528.4 & 24.2 & 98.7 & 12.9 & 360.1 & 3.3 \\
\hline 1310 & 28.42 & 0.029 & 0.0010 & 2166.4 & 20.7 & 98.9 & 16.9 & 360.0 & 3.3 \\
\hline FUSE & 28.59 & 0.032 & 0.0014 & 1118.9 & 10.7 & 98.5 & 15.4 & 360.8 & 3.4 \\
\hline TOTA & & & 10465.4 & 100.0 & & & & 359.3 & 3.4 \\
\hline PLAT & GE & & & & & & & 360.3 & 2.7 \\
\hline
\end{tabular}

Priestly pluton, granodiorite; 95MDw 108; biotite; $\mathrm{J}=.007509$

\begin{tabular}{|c|c|c|c|c|c|c|c|c|c|}
\hline \multicolumn{10}{|c|}{ Priestly pluton, granodiorite; 95MDw 108; biotite; J=.007509 } \\
\hline 760 & 50.26 & 0.111 & 0.0911 & 4.2 & 1.1 & 46.4 & 4.4 & 291.2 & 26.3 \\
\hline 900 & 31.69 & 0.017 & 0.0087 & 26.7 & 7.0 & 91.8 & 28.9 & 356.4 & 4.1 \\
\hline 1010 & 30.84 & 0.018 & 0.0044 & 38.0 & 9.9 & 95.8 & 28.0 & 361.3 & 3.8 \\
\hline 1090 & 30.75 & 0.029 & 0.0034 & 38.6 & 10.0 & 96.7 & 17.1 & 363.5 & 3.9 \\
\hline 1130 & 30.70 & 0.083 & 0.0024 & 43.4 & 11.3 & 97.7 & 5.9 & 366.4 & 3.6 \\
\hline 1230 & 30.18 & 0.073 & 0.0013 & 74.1 & 19.3 & 98.7 & 6.7 & 364.2 & 3.4 \\
\hline 1310 & 29.92 & 0.052 & 0.0008 & 124.6 & 32.5 & 99.2 & 9.3 & 363.0 & 3.3 \\
\hline FUSE & 30.45 & 0.059 & 0.0025 & 34.4 & 9.0 & 97.6 & 8.3 & 363.3 & 5.8 \\
\hline TOTAI & & & 384.0 & 100.0 & & & & 362.3 & 4.0 \\
\hline PLATE & $\mathrm{AGE}$ & & & & & & & 363.2 & 2.3 \\
\hline \multicolumn{10}{|c|}{ Russell Mtn. pluton; 95MDw80; biotite; $\mathrm{J}=.00755$} \\
\hline 760 & 31.44 & 0.014 & 0.0079 & 639.2 & 5.0 & 92.6 & 34.7 & 358.3 & 3.6 \\
\hline 900 & 32.11 & 0.005 & 0.0021 & 1722.9 & 13.5 & 98.0 & 98.7 & 384.4 & 3.5 \\
\hline 1010 & 32.40 & 0.006 & 0.0019 & 1649.9 & 13.0 & 98.2 & 85.6 & 388.3 & 3.5 \\
\hline 1090 & 32.71 & 0.004 & 0.0016 & 1721.9 & 13.5 & 98.5 & 116.0 & 392.8 & 3.6 \\
\hline 1130 & 32.62 & 0.005 & 0.0010 & 2327.8 & 18.3 & 99.1 & 97.3 & 393.8 & 3.5 \\
\hline 1230 & 32.44 & 0.006 & 0.0006 & 2508.1 & 19.7 & 99.3 & 81.1 & 392.9 & 3.6 \\
\hline 1310 & 32.53 & 0.008 & 0.0007 & 1530.2 & 12.0 & 99.3 & 65.1 & 393.9 & 3.6 \\
\hline FUSE & 32.66 & 0.011 & 0.0012 & 619.5 & 4.9 & 98.8 & 44.0 & 393.4 & 3.8 \\
\hline TOTAI & & & 12719.5 & 100.0 & & & & 389.7 & 3.6 \\
\hline PLATE & $\mathrm{AGE}$ & & & & & & & 393.4 & 3.0 \\
\hline \multicolumn{10}{|c|}{ Russell Mtn. pluton; 95MDw98; muscovite; $J=.007668$} \\
\hline 770 & 65.509 & 0.04333 & 0.12239 & 3.7 & 1.1 & 44.8 & 11.3 & 366.0 & 22.8 \\
\hline 870 & 38.853 & 0.01887 & 0.02433 & 9.3 & 2.7 & 81.5 & 26.0 & 392.0 & 6.3 \\
\hline 950 & 33.449 & 0.00837 & 0.00506 & 19.2 & 5.5 & 95.5 & 58.6 & 395.2 & 3.8 \\
\hline 1010 & 32.466 & 0.01020 & 0.00212 & 50.8 & 14.5 & 98.0 & 48.0 & 393.9 & 3.6 \\
\hline 1085 & 32.277 & 0.00489 & 0.00162 & 70.3 & 20.1 & 98.5 & 100.2 & 393.5 & 3.6 \\
\hline 1150 & 32.107 & 0.00234 & 0.00096 & 53.5 & 15.3 & 99.1 & 209.2 & 393.7 & 3.5 \\
\hline 1230 & 32.111 & 0.00589 & 0.00096 & 83.8 & 24.0 & 99.1 & 83.2 & 393.8 & 3.5 \\
\hline FUSE & 32.472 & 0.03211 & 0.00241 & 58.6 & 16.8 & 97.8 & 15.3 & 393.1 & 3.8 \\
\hline TOTAI & & & 349.3 & 100.0 & & & & 393.4 & 3.9 \\
\hline \multicolumn{3}{|c|}{ PLATEAU AGE } & & & & & & 393.6 & 2.7 \\
\hline
\end{tabular}


Table $5 .{ }^{40} \mathrm{Ar} /{ }^{39} \mathrm{Ar}$ data for plutonic rocks, Maine-Continued.

\begin{tabular}{|c|c|c|c|c|c|c|c|c|c|}
\hline Temp ${ }^{\circ} \mathrm{C}$ & ${ }^{40} \mathrm{Ar} /{ }^{3.9} \mathrm{Ar}$ & ${ }^{37} \mathrm{Ar} /{ }^{39} \mathrm{Ar}$ & ${ }^{36} \mathrm{Ar} /{ }^{39} \mathrm{Ar}$ & Moles ${ }^{39} \mathrm{Ar}^{*}$ & $\%$ total ${ }^{39} \mathrm{Ar}$ & $\begin{array}{l}\%{ }^{4 / 1} \mathrm{Ar} \\
\mathrm{rad}\end{array}$ & $\mathrm{K} / \mathrm{Ca}$ & $\begin{array}{l}\text { Apparent age } \\
\text { (Ma) }\end{array}$ & $\begin{array}{l}\text { Error } \\
(\mathrm{Ma})\end{array}$ \\
\hline \multicolumn{10}{|c|}{ Sebec Lake pluton, granodiorite; 95MDw71; muscovite; $\mathrm{J}=, 007477$} \\
\hline 850 & 35.78 & 0.020 & 0.02633 & 14.4 & 5.1 & 78.2 & 24.1 & 342.8 & 4.5 \\
\hline 950 & 32.19 & 0.031 & 0.00872 & 20.6 & 7.2 & 92.0 & 16.0 & 360.7 & 11.5 \\
\hline 1010 & 32.41 & 0.017 & 0.00436 & 34.4 & 12.0 & 96.0 & 28.3 & 377.2 & 3.6 \\
\hline 1090 & 32.31 & 0.021 & 0.00356 & 42.6 & 14.9 & 96.7 & 24.4 & 378.8 & 3.4 \\
\hline 1130 & 32.04 & 0.016 & 0.00317 & 39.4 & 13.8 & 97.0 & 30.9 & 377.1 & 3.4 \\
\hline 1210 & 31.77 & 0.017 & 0.00266 & 43.6 & 15.3 & 97.5 & 29.5 & 375.8 & 3.4 \\
\hline 1270 & 31.89 & 0.013 & 0.00167 & 45.2 & 15.9 & 98.4 & 38.9 & 380.3 & 3.5 \\
\hline FUSE & 31.42 & 0.019 & 0.00181 & 45.1 & 15.8 & 98.2 & 25.7 & 374.7 & 3.4 \\
\hline TOTAL & & & 285.4 & 100.0 & & & & 374.4 & 4.1 \\
\hline PLATEAU & AGE & & & & & & & 377.2 & 4.4 \\
\hline \multicolumn{10}{|c|}{ Sebec Lake pluton, granodiorite; $95 \mathrm{MDw} 72 ;$ biotite; $\mathrm{J}=.007885$} \\
\hline 750 & 15.616 & 0.00261 & 0.00658 & 88.8 & 14.4 & 87.4 & 187.4 & 184.5 & 1.9 \\
\hline 900 & 31.175 & 0.00810 & 0.00360 & 77.1 & 12.5 & 96.5 & 60.5 & 384.2 & 17.5 \\
\hline 1090 & 31.219 & 0.00277 & 0.00122 & 147.4 & 23.8 & 98.8 & 176.6 & 392.7 & 3.5 \\
\hline 1130 & 31.093 & 0.00313 & 0.00090 & 154.2 & 24.9 & 99.1 & 156.3 & 392.4 & 3.5 \\
\hline 1230 & 31.269 & 0.00634 & 0.00046 & 79.5 & 12.9 & 99.5 & 77.3 & 395.9 & 3.6 \\
\hline 1300 & 31.153 & 0.01663 & 0.00077 & 69.3 & 11.2 & 99.2 & 29.5 & 393.5 & 3.5 \\
\hline FUSE & 31.031 & 0.08028 & 0.01556 & 2.1 & 0.3 & 85.1 & 6.1 & 341.4 & 17.5 \\
\hline \multirow{3}{*}{\multicolumn{2}{|c|}{$\begin{array}{l}\text { TOTAL } \\
\text { PLATEAU AGE }\end{array}$}} & & 618.4 & 100.0 & & & & 362.0 & 5.1 \\
\hline & & & & & & & & 392.5 & 2.5 \\
\hline & & \multicolumn{8}{|c|}{ Seboeis pluton, diorite; $95 \mathrm{MDw} 135 \mathrm{~A}$; biotite; $\mathrm{J}=.007937$} \\
\hline 770 & 39.935 & 0.07719 & 0.07671 & 6.4 & 1.4 & 43.2 & 6.3 & 231.6 & 11.5 \\
\hline 870 & 33.444 & 0.02203 & 0.01627 & 26.3 & 5.8 & 85.6 & 22.2 & 369.3 & 4.2 \\
\hline 950 & 32.429 & 0.01091 & 0.00642 & 47.2 & 10.5 & 94.1 & 44.9 & 391.3 & 3.5 \\
\hline 1010 & 32.378 & 0.01232 & 0.00461 & 56.9 & 12.7 & 95.7 & 39.8 & 396.9 & 3.6 \\
\hline 1085 & 32.314 & 0.01106 & 0.00221 & 73.2 & 16.3 & 97.9 & 44.3 & 404.3 & 3.6 \\
\hline 1145 & 32.100 & 0.00865 & 0.00150 & 79.1 & 17.6 & 98.6 & 56.6 & 404.2 & 3.6 \\
\hline 1230 & 31.710 & 0.00868 & 0.00105 & 101.8 & 22.6 & 99.0 & 56.5 & 401.3 & 3.6 \\
\hline FUSE & 31.956 & 0.00836 & 0.00204 & 58.5 & 13.0 & 98.1 & 58.6 & 400.7 & 4.3 \\
\hline TOTAL & & 0.060 .0 & 449.5 & 100.0 & & & & 396.3 & 3.8 \\
\hline \multicolumn{10}{|c|}{ Seboeis pluton, granite; 95MDw135B; muscovite; J=.007465 } \\
\hline 760 & 46.10 & 0.030 & 0.04967 & 7.7 & 1.5 & 68.1 & 16.4 & 380.0 & 9.3 \\
\hline 900 & 35.11 & 0.008 & 0.00680 & 39.4 & 7.7 & 94.2 & 61.0 & 398.2 & 4.2 \\
\hline 1010 & 34.19 & 0.003 & 0.00263 & 95.5 & 18.8 & 97.7 & 183.5 & 401.6 & 3.6 \\
\hline 1090 & 34.02 & 0.007 & 0.00220 & 88.3 & 17.3 & 98.0 & 69.4 & 401.1 & 3.6 \\
\hline 1130 & 33.87 & 0.003 & 0.00143 & 95.0 & 18.7 & 98.7 & 162.4 & 401.9 & 3.6 \\
\hline 1230 & 33.80 & 0.004 & 0.00156 & 94.8 & 18.6 & 98.6 & 135.0 & 400.8 & 3.6 \\
\hline 1310 & 33.84 & 0.006 & 0.00153 & 57.8 & 11.3 & 98.6 & 137.9 & 401.3 & 3.6 \\
\hline FUSE & 33.44 & 0.013 & 0.00315 & 31.0 & 6.1 & 97.2 & 36.9 & 391.8 & 4.5 \\
\hline TOTAL & & & 509.4 & 100.0 & & & & 400.2 & 6.7 \\
\hline PLATEAU & AGE & & & & & & & 401.3 & 2.8 \\
\hline \multicolumn{10}{|c|}{ Seboeis pluton, granodiorite; 95MDw139B; biotite; $\mathrm{J}=.007437$} \\
\hline 760 & 47.15 & 0.068 & 0.0896 & 3.5 & 0.6 & 43.8 & 7.2 & 258.0 & 26.8 \\
\hline 900 & 32.15 & 0.015 & 0.0086 & 45.0 & 8.0 & 92.0 & 33.6 & 358.8 & 4.5 \\
\hline 1010 & 31.29 & 0.015 & 0.0044 & 73.8 & 13.2 & 95.8 & 33.2 & 363.0 & 3.7 \\
\hline 1090 & 31.03 & 0.012 & 0.0026 & 76.5 & 13.7 & 97.5 & 40.7 & 366.2 & 3.4 \\
\hline 1130 & 30.77 & 0.010 & 0.0019 & 94.4 & 16.9 & 98.2 & 50.0 & 365.6 & 3.7 \\
\hline 1230 & 30.49 & 0.008 & 0.0013 & 115.4 & 20.6 & 98.7 & 58.4 & 364.2 & 3.4 \\
\hline 1310 & 30.40 & 0.009 & 0.0013 & 106.3 & 19.0 & 98.7 & 56.2 & 363.2 & 4.0 \\
\hline FUSE & 30.86 & 0.015 & 0.0021 & 44.8 & 8.0 & 98.0 & 33.4 & 365.9 & 3.7 \\
\hline TOTAL & & & 559.8 & 100.0 & & & & 363.4 & 3.9 \\
\hline PLATEAU & AGE & & & & & & & 364.7 & 4.3 \\
\hline \multicolumn{10}{|c|}{ Shirley pluton; 95MDw90B; biotite; J=7.888001 $\times 10^{-3}$} \\
\hline 720 & 40.59 & 0.020 & 0.0529 & 7.5 & 0.9 & 61.4 & 24.8 & 323.9 & 15.9 \\
\hline 900 & 31.36 & 0.005 & 0.0038 & 107.4 & 12.5 & 96.4 & 103.0 & 385.7 & 3.6 \\
\hline 1010 & 31.15 & 0.007 & 0.0019 & 135.5 & 15.8 & 98.2 & 74.9 & 389.9 & 3.6 \\
\hline 1090 & 31.13 & 0.008 & 0.0015 & 129.7 & 15.1 & 98.5 & 63.0 & 391.0 & 3.5 \\
\hline 1130 & 30.86 & 0.007 & 0.0009 & 178.2 & 20.8 & 99.1 & 75.2 & 389.8 & 3.5 \\
\hline 1230 & 30.91 & 0.011 & 0.0008 & 167.2 & 19.5 & 99.2 & 45.3 & 390.6 & 3.6 \\
\hline 1310 & 31.11 & 0.028 & 0.0009 & 83.1 & 9.7 & 99.1 & 17.3 & 392.8 & 3.7 \\
\hline FUSE & 31.38 & 0.036 & 0.0018 & 49.0 & 5.7 & 98.3 & 13.8 & 392.8 & 4.1 \\
\hline TOTAL & & & 857.6 & 100.0 & & & & 389.5 & 3.7 \\
\hline \multicolumn{3}{|c|}{ PLATEAU AGE; } & & & & & & 390.3 & 3.0 \\
\hline mole & $1.5 \times 10$ & & & & & & & & \\
\hline
\end{tabular}


DE, fig. 6; A. Ludman, written commun., 1998). Both of these plutons have been dated by the U-Pb zircon method, but both ages have fairly large errors.

A cluster of seven previously dated plutons in the Eastern Townships of Québec and adjacent Maine fall within the Givetian to Famennian age range. Those plutons dated by the

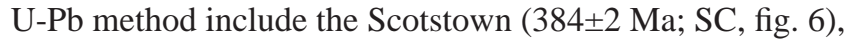
Lac aux Araignées (in Québec, or Spider Lake, in Maine;

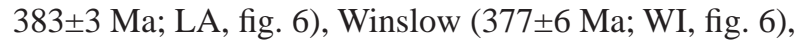
Aylmer (375 \pm 3 Ma; AY, fig. 6), and Ste.-Cécile (374 1 Ma; ST, fig. 6; Simonetti and Doig, 1990). Plutons in this group dated by the ${ }^{40} \mathrm{Ar} /{ }^{39} \mathrm{Ar}$ method include the Chain of Ponds (373.3 $\pm 2 \mathrm{Ma}$; $\mathrm{CH}$, fig. 6) and Big Island Pond plutons

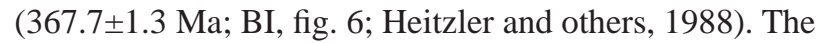
newly dated Hog Island and Beaver Cove, discussed below, appear to belong in this group. Three other new ages from the Priestly, Deboullie, and Chandler plutons, also discussed below, are slightly younger but occur within the same general strike belt. Five previously dated plutons of Famennian age form a belt near the coast. In Maine, these plutons include the Waldoboro pluton (368 \pm 2 Ma; WD, fig. 6), the Mount Waldo pluton (371 $\pm 2 \mathrm{Ma}$; MW, fig. 6), and two small pegmatite bodies dated at $367 \pm 1$ and $371 \pm 1 \mathrm{Ma}$ (GA, GR, fig. 6; Tucker and others, in press). (All ages are U-Pb zircon ages.) Similarly, M.L. Bevier (written commun., 1996) reported a U-Pb age of 366.5 \pm 1 Ma from the Mount Douglas pluton in southern New Brunswick (MD, fig. 6).

\section{Center Pond Pluton}

The Center Pond pluton (CP, fig. 6) is a hornblendebearing granodiorite that intrudes steeply dipping, calcareous siltstone of Silurian age (Scambos, 1980). Two concordant zircon analyses yielded a mean ${ }^{207} \mathrm{~Pb} /{ }^{206} \mathrm{~Pb}$ age of $377 \pm 3 \mathrm{Ma}$ (fig. 7). The pluton is cut in half and offset dextrally about $2 \mathrm{~km}$ by the Center Pond fault (Scambos, 1980). A biotite separate from a penetratively dextrally sheared granitoid near the fault yielded an ${ }^{40} \mathrm{Ar} /{ }^{39} \mathrm{Ar}$ age of $368.7 \pm 2.9 \mathrm{Ma}$ (fig. 8), which may date the strike-slip event.

\section{Hammond Ridge Sill}

At Hammond Ridge in the aureole of the Katahdin pluton (HM, fig. 6), a 50-cm-thick, tabular, concordant body of felsite interlayered with hornfels of the Carrabassett Formation yielded five concordant zircon fractions with a mean ${ }^{207} \mathrm{~Pb} /{ }^{206} \mathrm{~Pb}$ age of $377.2 \pm 1.5 \mathrm{Ma}$ (fig. 7). This sill was at first mistaken for an ash bed that would have dated the Carrabassett Formation (the reason why we analyzed so many fractions), but its young age requires that it be a sill.

\section{Hog Island Pluton}

The Hog Island pluton (HI, fig. 6) intrudes Ordovician granodiorite, Silurian calcareous rocks, and Devonian flysch of the Seboomook Group at the north end of the Boundary
Mountains anticlinorium. The pluton was mapped by Albee and Boudette (1972), who interpreted the contact metamorphism as either synregional or postregional metamorphism. A metapelite that contains biotite and cordierite, both formed during the contact-metamorphic event (the regional metamorphic grade is chlorite), is shown in figure $9 D$. Cordierite porphyroblasts overgrew a weak foliation, which, we suggest, formed during an earlier Acadian deformation; a second foliation is defined by aligned biotite, some of which occurs in strain shadows around cordierite. Although the Hog Island pluton is probably much younger than the first Acadian deformation, its emplacement was nonetheless synkinematic. A single zircon fraction yielded a concordant ${ }^{207} \mathrm{~Pb} /{ }^{206} \mathrm{~Pb}$ age of $371 \pm 3$ (fig. 7); biotite yielded an ${ }^{40} \mathrm{Ar} /{ }^{39} \mathrm{Ar}$ plateau age of 368.6 \pm 3.1 Ma (fig. 8).

\section{Beaver Cove Pluton}

The Beaver Cove pluton (BC, fig. 6) is a small, unfoliated stock of leucocratic biotite granodiorite surrounded by the Moxie pluton. We obtained an ${ }^{40} \mathrm{Ar} /{ }^{39} \mathrm{Ar}$ plateau age of 372.4 $\pm 2.7 \mathrm{Ma}$ from a biotite separate (fig. 8), suggesting that the Beaver Cove pluton is as much as 34 m.y. younger than the Moxie pluton and unrelated to it (see Gabis and others, 1994).

\section{Deboullie Pluton}

The Deboullie pluton (DB, fig. 6) is a composite body that includes syenite, granodiorite, and monzonite phases (Boone, 1958). Boone's mapping showed that the pluton truncates steeply dipping bedding and cleavage in the Seboomook Group and so postdates the main regional cleavage. Textures from the contact aureole, however, indicate that plutonism was followed by some additional shortening: Contact-metamorphic biotite is cut by a strong pressure-solution cleavage that parallels the regional fabric. Two samples of pink monzonite yielded ${ }^{40} \mathrm{Ar} /{ }^{39} \mathrm{Ar}$ plateau ages of $365.4 \pm 3.2$ and 363.2 $\pm 3.0 \mathrm{Ma}$ on biotite. Similarly, biotite from granodiorite yielded an ${ }^{40} \mathrm{Ar} /{ }^{39} \mathrm{Ar}$ age of $364.2 \pm 3.2$ (fig. 8).

\section{Priestly Pluton}

The Priestly pluton (PR, fig. 6), a stock of unfoliated biotite-hornblende granodiorite, intrudes flysch of the Seboomook Group (Boudette and others, 1976). Textures in the contact aureole suggest that the pluton was emplaced into already-foliated slate. At the north end of the pluton, cordierite porphyroblasts contain inclusion trails of an older slaty cleavage (fig. 9E). Biotite is randomly oriented and indicates static conditions of contact metamorphism; cordierite porphyroblasts grew preferentially in the direction of the older foliation, apparently also under static metamorphic conditions. A single zircon fraction yielded a concordant ${ }^{207} \mathrm{~Pb} /{ }^{206} \mathrm{~Pb}$ age of $360.5 \pm 3.3$ (fig. 7). ${ }^{40} \mathrm{Ar} /{ }^{39} \mathrm{Ar}$ plateau ages of $363.2 \pm 2.3$ and $360.3 \pm 2.7 \mathrm{Ma}$ were obtained on biotite (fig. 8). 


\section{Chandler Pluton}

The Chandler pluton ( $\mathrm{CH}$, fig. 6) intrudes flysch of the Seboomook Group and Ordovician mafic igneous rocks along the eastern limb of the Munsungun anticlinorium. As far as we are aware, the pluton and surrounding rocks have never been mapped in detail, and outcrop is not plentiful. A total-gas ${ }^{40} \mathrm{Ar} /{ }^{39} \mathrm{Ar}$ age of $366.5 \pm 3.7 \mathrm{Ma}$ was obtained on biotite (fig. 8); the age spectrum is slightly hump shaped.

\section{Seboeis Pluton}

As mentioned above, although the Seboeis pluton is shown on the "Geologic Map of Maine" (Osberg and others, 1985) as a single body, it appears to include granodiorite, granite, and diorite of Emsian age, as well as a much younger phase. At East Branch Lake (SE, fig. 6), an unfoliated biotite granodiorite yielded an ${ }^{40} \mathrm{Ar} /{ }^{39} \mathrm{Ar}$ plateau age of $364.7 \pm 4.3 \mathrm{Ma}$ (fig. 8).

\section{The Silurian-Devonian Time Scale: Basis for Correlations Between Paleontologically and Isotopically Dated Events}

We are able to make precise - and, in some cases, unexpected-correlations between isotopically and paleontologically dated events because of a parallel effort to improve the Silurian-Devonian time scale (Tucker and others, 1998). New tiepoints require significant changes to previous time scales; each tiepoint is based on replicate, concordant, isotopedilution $\mathrm{U}-\mathrm{Pb}$ zircon analyses of ashes or volcanic strata, dated to within one or two conodont zones. The key dates are as follows: (1) a concordant U-Pb zircon age of 417.6 $\pm 1.0 \mathrm{Ma}$ on the Bald Hill ash from the early Lochkovian Kalkberg Formation of eastern New York; (2) a concordant U-Pb zircon age of $408.3 \pm 1.9 \mathrm{Ma}$ on the Sprout Brook ash from the early Emsian Esopus Formation of eastern York; (3) a concordant $\mathrm{U}-\mathrm{Pb}$ zircon age of $391.4 \pm 1.8 \mathrm{Ma}$ on the Eifelian Tioga ash of Wytheville, Va., confirming the concordant $\mathrm{U}-\mathrm{Pb}$ monazite age of 390.0 \pm 0.5 Ma reported for the Tioga ash of Pennsylvania by Roden and others (1990); (4) a concordant U-Pb zircon age of 381.1 $\pm 1.3 \mathrm{Ma}$ on the Little War Gap ash from the Frasnian part of the Chattanooga Shale of eastern Tennessee; and (5 and 6) concordant $\mathrm{U}-\mathrm{Pb}$ zircon ages of $363.8 \pm 2.2$ and $363.4 \pm 1.8$ Ma on rhyolite that bracket a closely dated palyniferous horizon in the Famennian Piskahegan Group of New Brunswick. The vertical axis in figure 4 is numerically calibrated according to the new time scale of Tucker and others (1998), which is contrasted with that of Harland and others (1990) in table 6.

These revisions are critical to the present study. Using the new time scale, most of the closely dated plutons listed in table 3 are now correlated with a younger stage than they would be on the time scale of Harland and others (1990). Among the plutons that are critical to positioning the deformation front,
Table 6.-Comparison of time scales.

[All values in millions of years before present $(\mathrm{Ma})$ ]

\begin{tabular}{lll}
\hline $\begin{array}{c}\text { Stage or series } \\
\text { boundary }\end{array}$ & $\begin{array}{c}\text { Harland and } \\
\text { others } \\
(1990)\end{array}$ & $\begin{array}{c}\text { Tucker and } \\
\text { others } \\
(1998)\end{array}$ \\
\hline $\begin{array}{c}\text { Base of Tournaisian } \\
\text { (= base of Carboniferous). }\end{array}$ & 362.5 & 362 \\
Base of Famennian & 367.0 & 376.5 \\
Base of Frasnian- & 377.4 & 382.5 \\
Base of Givetian & 380.8 & 387.5 \\
Base of Eifelian- & 386.0 & 394 \\
Base of Emsian & 390.4 & 409.5 \\
Base of Pragian & 396.3 & 413.5 \\
Base of Lochkovian & 408.5 & 418 \\
(= base of Devonian). & 410.7 & 419 \\
Base of Pridoli & & 424 \\
Base of Ludlow - & 424.0 & \\
\hline
\end{tabular}

the new time scale shows that (1) the 424- to 417-Ma plutons in southern Maine are Ludlow to Lochkovian rather than Ludlow only, (2) the 404-408 plutons of interior Maine are Emsian rather than Lochkovian, and (3) the Scotstown pluton of the Eastern Townships is Givetian rather than Eifelian. Looking at it another way, the new time scale implies that the Sebec Lake, Onawa, Russell Mountain, and Blanchard plutons are the same age as the Tomhegan Formation, and not the Beck Pond Limestone, as implied by the Harland and others (1990) time scale. Paleogeographic maps based on the old time scale would have been fraught with such errors.

\section{Location Through Time of the Acadian Deformation Front and Foreland Basin}

In this section, we synthesize the foregoing results with the aid of two maps (figs. 10,11) that track the migration of the leading edge of the foreland basin and of the deformation front. Seven time slices are depicted, each spanning a few million years. The gross paleogeography and key stratigraphic localities are shown in figure 12 for the four best-constrained time slices.

In constructing these figures, we interpolated the leading edge of the foreland basin at a given time between the positions of foreland-basin and far-foreland deposits of that age. Likewise, the deformation front was interpolated between fossiliferous foreland-basin deposits laid down before regional deformation and dated plutons that cut Acadian structures. In some places, as discussed below, figures 10 and 11 were used interdependently to help fill in gaps in knowledge; mostly, however, these two maps are based on independent data that nonetheless lead to the same general conclusions. For the purposes of figure 10, the details of locally complex Acadian deformation histories are not crucial because the point is merely to locate a boundary between rocks that had already undergone at least some contractional deformation from those that still had not. Likewise, unresolved aspects of the pretectonic, syntectonic, or posttectonic nature of various plutons 
are not crucial for present purposes, so long as evidence exists that some regional deformation occurred in a particular plutonic belt before pluton emplacement.

\section{Palinspastic Considerations}

The key localities are plotted on a present-day base map; no attempt has been made to remove the effects of Acadian folding and thrust faulting, post-Acadian shortening, or dextral displacement across the Norumbega or other strike-slip faults. We originally had intended to use a palinspastic base, but we found it impossible to construct such a map on the basis of published maps and cross sections. There are simply too few good marker units to unravel the structure, and the exposure in Maine is too poor.

We can, however, make three comments in support of our use of the present-day base. First, none of the rocks in the study area (fig. 1) has been displaced enormous, immeasurable distances across terrane-bounding faults since the beginning of the Ludlow. By that time, the Pocomoonshine pluton had linked the Fredericton Basin with the St. Croix belt (West

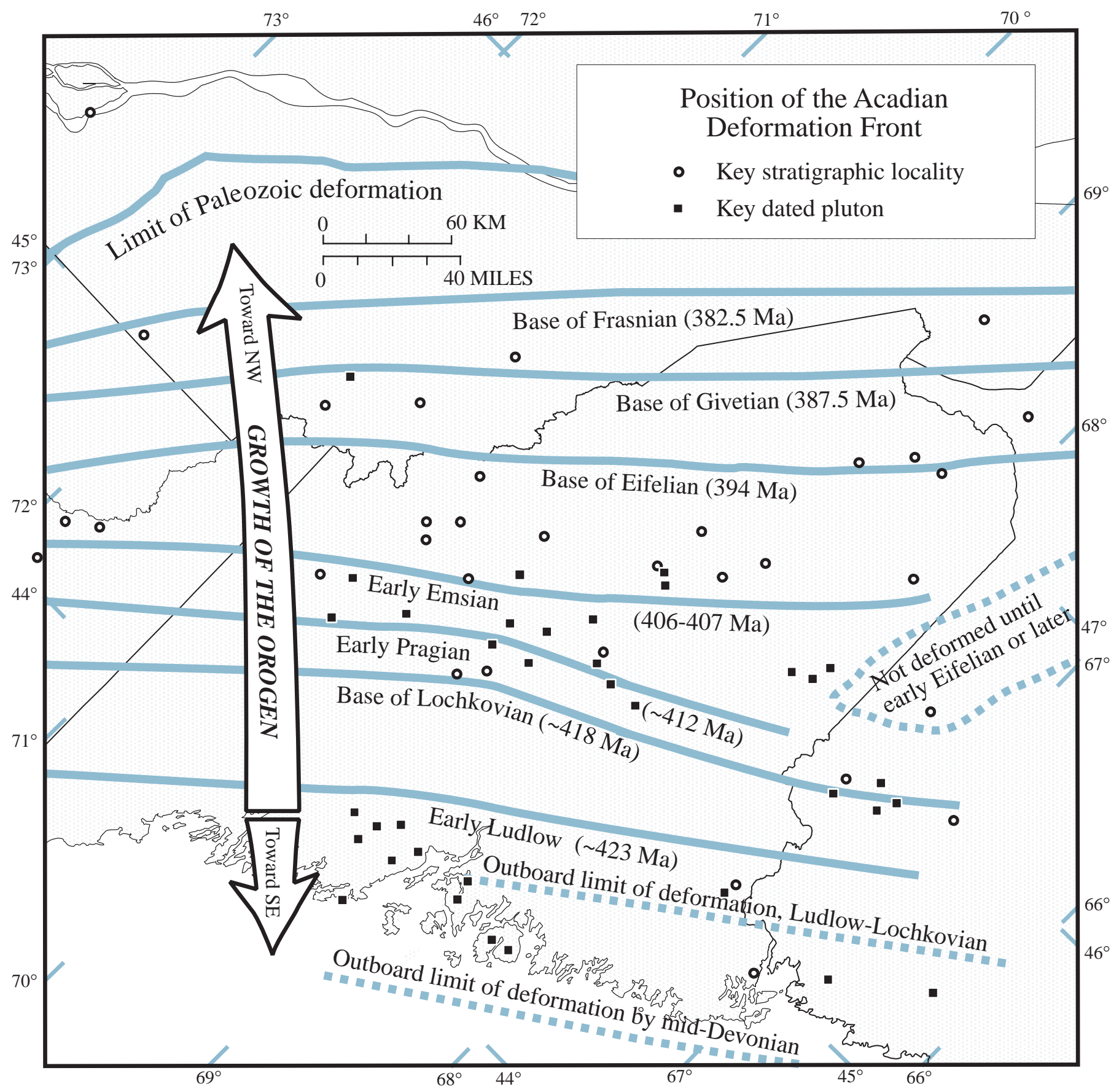

Figure 10.-Maine and adjacent parts of New Brunswick, Québec, New Hampshire, and Vermont, showing successive positions of Acadian deformation front as it migrated across the northern Appalachians. 
and others, 1992), which, in turn, was linked by clasts in the Silurian Oak Bay conglomerate to the Coastal volcanic belt (loc. 43, fig. 1; Ludman, 1981). The Fredericton Basin and Miramichi anticlinorium were linked by conglomerate clasts in the Taxis River beds by the Early Silurian (loc. 36, fig. 1). The Aroostook-Matapedia Basin was two sided because it has provenance linkages to anticlinoria to both the west and east (Berry and Osberg, 1989; Hopeck, 1991). Specifically, the Llandovery Frenchville Formation near the basin's western margin was derived from the Pennington anticlinorium just to the west (loc. 25, fig. 1; Roy and Mencher, 1976), whereas the conglomeratic Daggett Ridge Formation (probably Late Ordovician to middle Silurian; Ludman, 1990) on the eastern margin was derived from the Miramichi anticlinorium (loc. 36, fig. 1). The Central Maine Basin is linked stratigraphically to older rocks on the northwest; specifically, conglomerate in the Llandovery Rangeley Formation (loc. 26, fig. 1) includes clasts traced to the Attean pluton along the Boundary Mountains anticlinorium (Moench and Pankiwskyj, 1988). Finally, the Connecticut Valley-Gaspé Basin can be

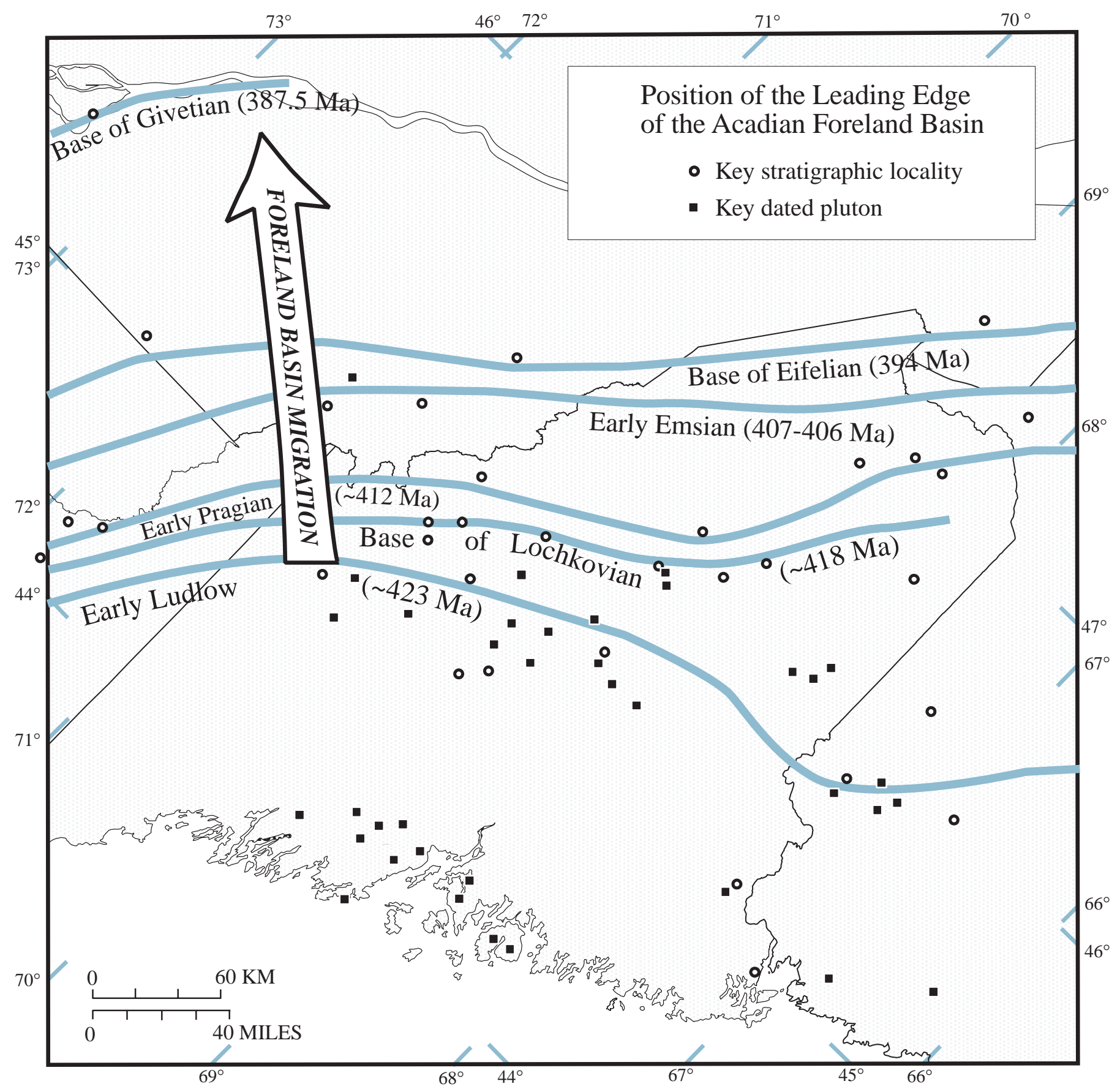

Figure 11.-Maine and adjacent parts of New Brunswick, Québec, New Hampshire, and Vermont, showing successive positions of leading edge of the Acadian foreland basin, constructed by following approach used by Bradley (1989) for the Taconic foreland basin. 
seen as a foreshortened, two-sided basin because (1) Late Ordovician to Early Silurian conglomerate of the Cabano and Depot Mountain Formations along its northwestern margin in Québec and northernmost Maine was derived from Taconic highlands that lay to the northwest (loc. 6, fig. 1; Roy, 1989) and (2) along the opposite basin margin in northern Maine, the Fish River Lake Formation includes sandstone and conglomerate that can be traced to sources in the Pennington anticlinorium, just to the east (loc. 21, fig. 1; Boone, 1970). Although the contact between the Central Maine and Aroostook-Matapedia Basins is probably a major thrust fault (A. Ludman, written commun., 1998), the two basins shared a common northwesterly source region during the Silurian and so are not exotic relative to each other. Similarly, the Central Maine and Fredericton Basins are in fault contact and may not have been continuous with each other, as some workers
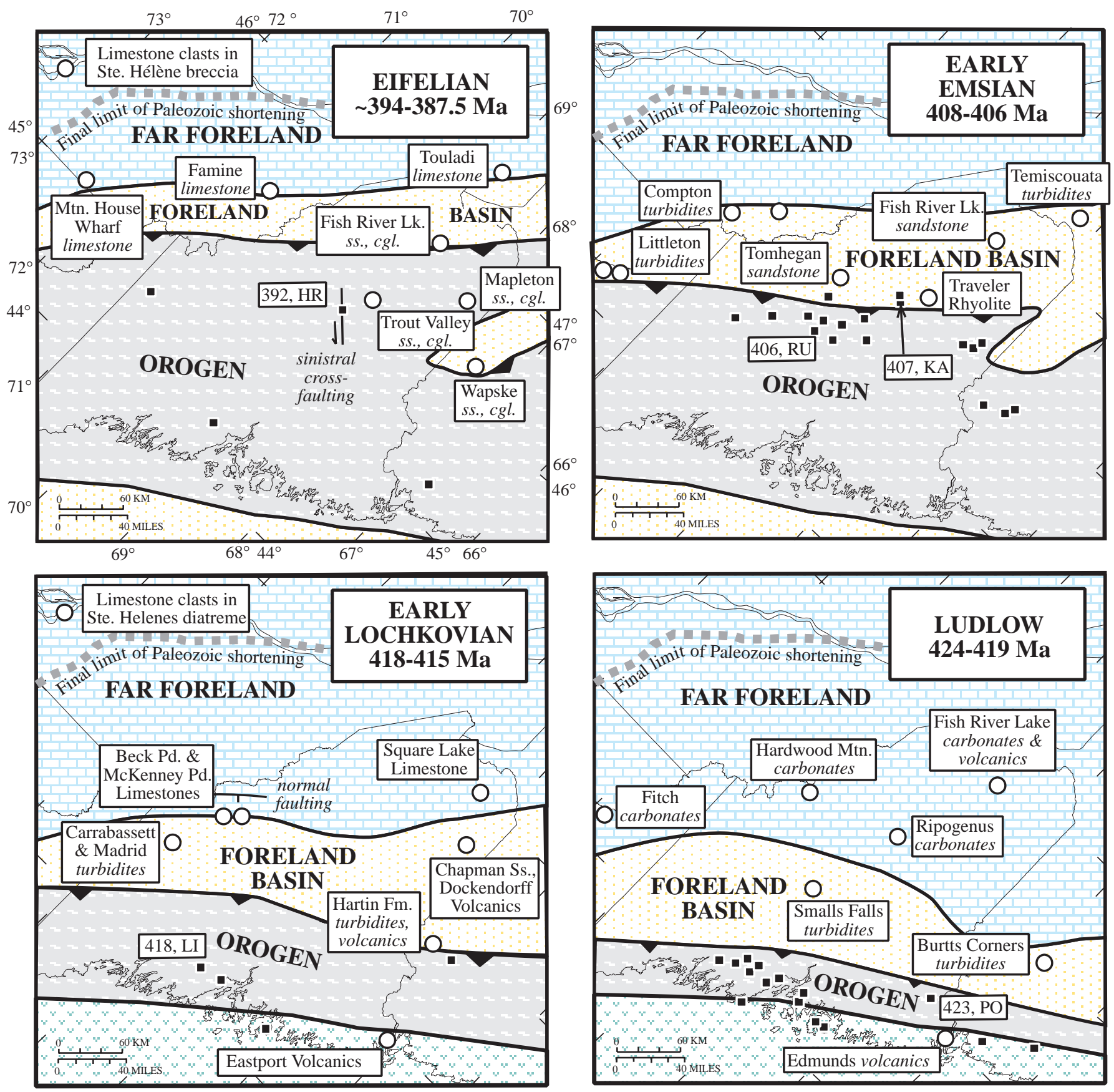

Figure 12.-Schematic, nonpalinspastic paleogeographic maps of study area (see fig. 1), showing migration of orogen and foreland basin during four time slices. Circles, locations of fossils in stratified rocks of appropriate age range, except for the Traveler Rhyolite, which is dated isotopically; black squares, locations of plutons in appropriate age range. Boxes show age and two-letter abbreviation for a few selected plutons, keyed to figure 6 and table 3. Toothed line denotes Acadian deformation front, which probably was a blind thrust rather than an emergent thrust during much of Late Silurian to Middle Devonian time. Paleogeography during the Eifelian and Emsian is unknown for area south of orogen (present coordinates). 
Table 7.-Data used to estimate Acadian and younger shortening in figure 13.

\begin{tabular}{|c|c|c|c|c|c|c|c|c|}
\hline $\begin{array}{l}\text { Map letter } \\
\text { (fig. 15) }\end{array}$ & $\begin{array}{l}\text { Azimuth } \\
\text { of cross } \\
\text { section }\end{array}$ & Location & Stretch & $\begin{array}{l}\text { 1/stretch } \\
\text { (plotted in } \\
\text { fig. 13) }\end{array}$ & $\begin{array}{l}\text { Final } \\
\text { width } \\
(\mathbf{k m})\end{array}$ & $\begin{array}{l}\text { Initial } \\
\text { width } \\
(\mathbf{k m})\end{array}$ & Notes & Reference \\
\hline $\mathbf{A}$ & $130^{\circ}$ & $\begin{array}{l}\text { Spencer Stream } \\
15^{\prime} \text { quadrangle }\end{array}$ & 0.75 & 1.33 & 6.5 & 8.6 & $\begin{array}{l}\text { Folded contact at base of the Seboomook } \\
\text { Group. }\end{array}$ & $\begin{array}{l}\text { Boucot and Heath } \\
(1969, \text { pl. 14), part } \\
\text { of section } U-U^{\prime}\end{array}$ \\
\hline B & $133^{\circ}$ & $\begin{array}{l}\text { Long Pond and } \\
\text { Brassua Lake } \\
15^{\prime} \text { quadrangles }\end{array}$ & .84 & 1.19 & 18.4 & 21.9 & $\begin{array}{l}\text { Folded contact between the Seboomook } \\
\text { Group and the overlying Tarratine } \\
\text { Formation. }\end{array}$ & $\begin{array}{l}\text { Boucot and Heath } \\
\text { (1969, pl. 14), part } \\
\text { of section L-L'. }\end{array}$ \\
\hline $\mathrm{C}$ & $143^{\circ}$ & $\begin{array}{l}\text { Brassua Lake } 15^{\prime} \\
\text { quadrangle }\end{array}$ & .68 & 1.47 & 6.8 & 10 & $\begin{array}{l}\text { Folded contact between the Tarratine } \\
\text { Formation and the overlying Tomhe gan } \\
\text { Formation (including the Kineo } \\
\text { Rhyolite). }\end{array}$ & $\begin{array}{l}\text { Boucot and Heath } \\
\text { (1969, pl. 14), part } \\
\text { of section F-F'. }\end{array}$ \\
\hline D & $090^{\circ}$ & $\begin{array}{l}\text { Caucomgomoc } \\
\text { Lake 15' } \\
\text { quadrangle }\end{array}$ & .55 & 1.82 & 9.6 & 17.5 & $\begin{array}{l}\text { Folded contact between the Northeast } \\
\text { Carry Formation and overlying strata of } \\
\text { the undivided part of the Seboomook } \\
\text { Group. }\end{array}$ & $\begin{array}{l}\text { Pollock (1985), part } \\
\text { of section D-D'. }\end{array}$ \\
\hline $\mathbf{E}$ & $100^{\circ}$ & $\begin{array}{l}\text { Lobster anticline } \\
\text { and Roach River } \\
\text { syncline }\end{array}$ & .77 & 1.30 & 18.9 & 24.6 & Folded contact at base of Silurian rocks. & $\begin{array}{l}\text { Boucot and Heath } \\
\text { (1969, pl. 14), part } \\
\text { of section D1-D1'. }\end{array}$ \\
\hline $\mathbf{F}$ & $129^{\circ}$ & $\begin{array}{l}\text { Munsungun } \\
\text { anticlinorium }\end{array}$ & 51 & 1.96 & 11.2 & 21.8 & $\begin{array}{l}\text { Folded base of the Spider Lake Volcan- } \\
\text { ics. }\end{array}$ & $\begin{array}{l}\text { Hall (1970), part of } \\
\text { section A-A? }\end{array}$ \\
\hline G & $140^{\circ}$ & $\begin{array}{l}\text { Phillips } 15^{\prime} \\
\text { quadrangle }\end{array}$ & .47 & 2.13 & 5.3 & 11.3 & $\begin{array}{l}\text { Folded contact between the Smalls Falls } \\
\text { and the Madrid and Carrabassett For- } \\
\text { mations. }\end{array}$ & $\begin{array}{l}\text { Moench (1971), } \\
\text { section B-B'. }\end{array}$ \\
\hline $\mathbf{H}$ & $140^{\circ}$ & $\begin{array}{l}\text { Kingsbury } 15^{\prime} \\
\text { quadrangle }\end{array}$ & .38 & 2.63 & 5.4 & 14.1 & $\begin{array}{l}\text { Folded contact between the Madrid and } \\
\text { Carrabassett Formations. Ignoring par- } \\
\text { asitic folds, stretch is } 0.49 \mathrm{~km} \text {, and initial } \\
\text { width is } 10.9 \mathrm{~km} \text {. }\end{array}$ & $\begin{array}{l}\text { Ludman (1978), part } \\
\text { of section } A-A^{\prime} \text {. }\end{array}$ \\
\hline I & $138^{\circ}$ & $\begin{array}{l}\text { Shin Pond } 15^{\prime} \\
\text { quadrangle }\end{array}$ & .77 & 1.30 & 13.8 & 17.8 & $\begin{array}{l}\text { Folded contact between unnamed Silu- } \\
\text { rian strata and the Seboomook Group. }\end{array}$ & $\begin{array}{l}\text { Neumann (1967), part } \\
\text { of section A-A'. }\end{array}$ \\
\hline $\mathbf{J}$ & $145^{\circ}$ & $\begin{array}{l}\text { Island Fails } 15^{\prime} \\
\text { quadrangle }\end{array}$ & .34 & 2.94 & 5.6 & 16.5 & $\begin{array}{l}\text { Folded contact between the Mattawam- } \\
\text { keag Formation and the overlying } \\
\text { Allsbury Formation (to the west) and } \\
\text { "rocks of Island Falls" (to the east). }\end{array}$ & $\begin{array}{l}\text { Eckren and Frischknecht } \\
(1967), \text { part of section } \\
\text { A-A'. }\end{array}$ \\
\hline$K$ & $150^{\circ}$ & $\begin{array}{l}\text { Maple and Hovey } \\
\text { Mountain area }\end{array}$ & .58 & 1.72 & .9 & 1.6 & $\begin{array}{l}\text { Folded contact of base of manganifer ous } \\
\text { beds. Constrained by exploratory } \\
\text { drilling. }\end{array}$ & $\begin{array}{l}\text { Pavlides (1962), section } \\
\text { A-A'. }\end{array}$ \\
\hline $\mathrm{L}$ & $090^{\circ}$ & $\begin{array}{l}\text { Presque Isle } 15^{\prime} \\
\text { quadrangle }\end{array}$ & .71 & 1.41 & 8.7 & 12.25 & $\begin{array}{l}\text { Weighted average of measurements } \\
\text { across the Chapman syncline (base of } \\
\text { unnamed Silurian limestone) and the } \\
\text { Dudley syncline (base of the Perham } \\
\text { Formation). Post-Mapleton stretch is } \\
0.86 \mathrm{~km} \text {. }\end{array}$ & $\begin{array}{l}\text { Boucot and others (1964), } \\
\text { parts of section A-B. }\end{array}$ \\
\hline
\end{tabular}

have suggested (McKerrow and Ziegler, 1971; Bradley, 1983). Nonetheless, they are linked by the chain of provenance relationships discussed above.

Second, evidence for the amount of fold-related Acadian shortening can be gleaned from some 1:62,500-scale quadrangle maps. We estimated shortening on 15 cross sections that were selected because they show at least one readily mapped contact, such as a carbonate-flysch contact. Using the sinuous-bed-length method, the ratio of the palinspastic crosssectional length to length in the deformed state was found to range from 1.30 to 2.78 (fig. 13), with a mean of 1.83 . These results, if taken as being representative of the entire orogen, suggest that the pre-Acadian across-strike width of figure 1 north of the Sennebec Pond fault was about twice the present width. The actual initial width was most likely even greater because we were able to estimate shortening only for those exceptional places with simple, traceable structure. In the southeasterly part of the Central Maine Basin in Maine (Osberg, 1988) and in the northwestern part of the same basin in New Hampshire (Eusden and others, 1996), early recumbent folds imply much greater amounts of shortening. Our estimate of shortening also does not account for the effects of penetrative strain.

Third, the only potentially problematic post-Acadian strike-slip fault in the study area (fig. 1) is the Devonian to
Carboniferous Norumbega Fault System. The displacement on this fault system may be large, but not so large that the rocks on either side are unrelated. Near the Maine-New Brunswick border, turbidites assigned to the Lower to middle Silurian Flume Ridge Formation occur at the same chlorite grade both northwest and southeast of the fault (Ludman, 1981). Map relations reviewed by Ludman and West (1999) suggest 125 to $140 \mathrm{~km}$ of cumulative dextral displacement across the Norumbega Fault System.

In summary, the successive deformation fronts mapped in figure 10 are probably more than twice as close together as they would be on an accurate palinspastic base. Also, localities outboard of the Norumbega Fault System were probably about 125 to $140 \mathrm{~km}$ farther northeast during the Acadian orogeny. Because the Norumbega Fault System approximately parallels Late Silurian paleogeographic belts, however, it does not significantly affect any of the conclusions presented below.

\section{Early Ludlow}

The Acadian orogeny was underway by the beginning of the Ludlow. The deformation front in figure 10 was placed between the posttectonic Pocomoonshine pluton at locality 37 (fig. 1) and the graptolite-bearing Smalls Falls Formation and 
Burtts Corners beds at localities 29 and 35 . The boundary between the foreland basin and far foreland was placed northwest of localities 29 and 35 but southeast of such shallowmarine carbonate units as the Hardwood Mountain, Spider Lake, Ripogenus, Grand Lake Seboeis, and Fish River Lake Formations (at locs. 11, 18, 16, 20, and 21, respectively).

Although our main emphasis in this report has been on tracking the northwestward migration of the Acadian deformation front, we also note evidence that a second, outboard boundary between deformed and undeformed rocks migrated a much shorter distance to the southeast. In the Coastal volcanic belt, volcanism and sedimentation continued without interruption through most of the Silurian and into the Lochkovian. The southeast boundary between deformed and undeformed rocks must have lain somewhere between the Pocomoonshine pluton and locality 38 (fig. 1) by early Ludlow time. It does not appear to have moved southeast until after Lochkovian time - that is, after deposition of the youngest Acadian-deformed strata at Eastport (loc. 38).

\section{Early Lochkovian}

During early Lochkovian time, the Acadian deformation front was located along the northwest flank of the Central Maine Basin and near the eastern margin of the AroostookMatapedia Basin. Its position is bracketed by foreland-basin and far-foreland strata to the northwest and syntectonic and posttectonic plutons to the southeast. Plutonic rocks within the range 418.5 to $415 \mathrm{Ma}$ delimit the zone of active deformation; these rocks include the syntectonic Lincoln sill (417 Ma) and the Hartfield pluton (415 Ma).

A Lochkovian foreland basin flanked the plutonic belt on the northwest. Strata assigned to this stage of the migrating foreland basin include the Hartin Formation (loc. 34, fig. 1), the Chapman Sandstone and Swanback Formation (loc. 31), a part of the Millimigassett Lake Formation (loc. 20), and the Madrid and Carrabassett Formations in the Central Maine Basin (for example, at locs. 26 and 30). The early Lochkovian position of the leading edge of the foreland basin (fig. 11) is

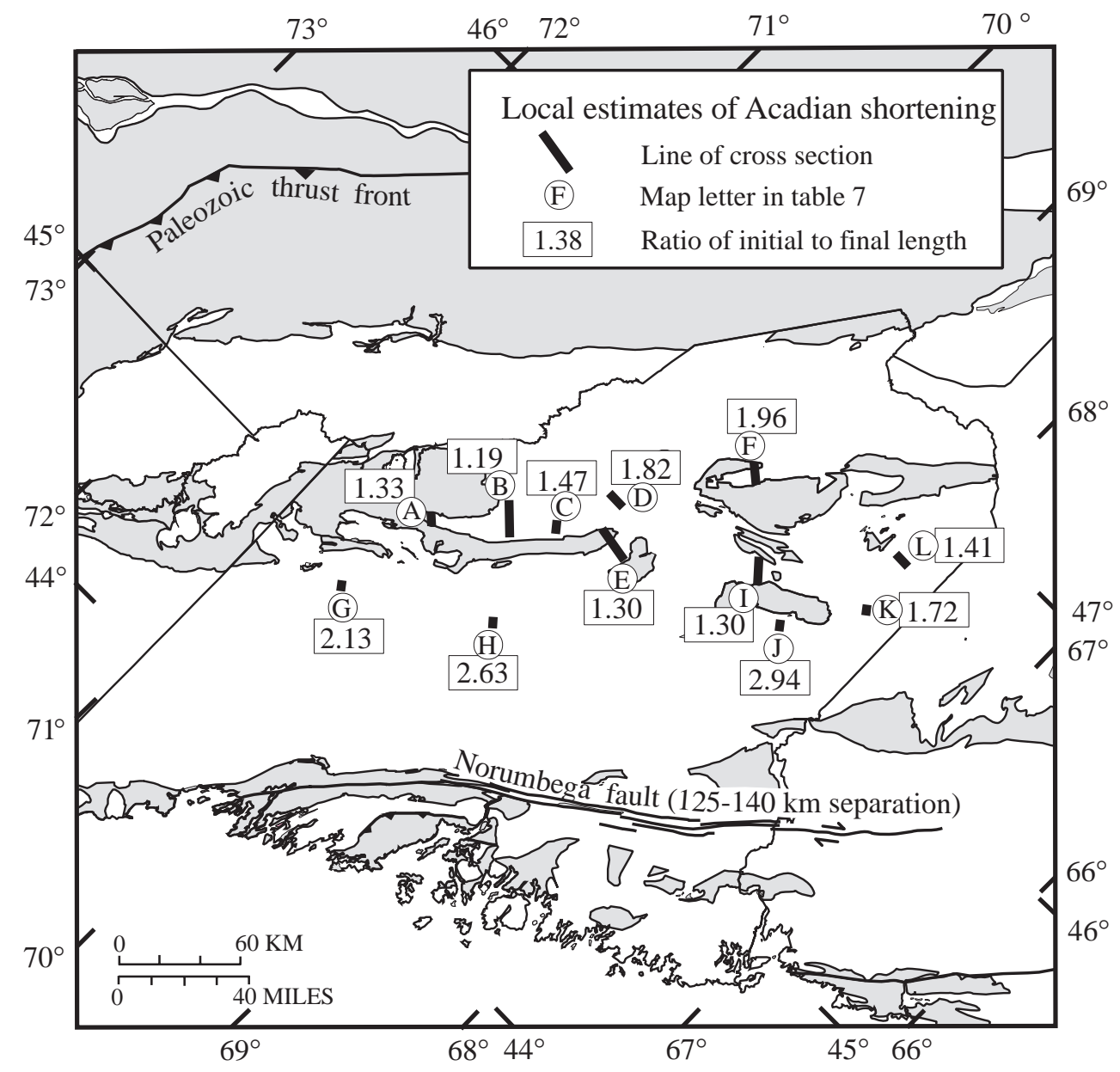

Figure 13.-Maine and adjacent parts of New Brunswick, Québec, New Hampshire, and Vermont, showing local estimates of Acadian and younger shortening, determined by sinuous-bed-length method, across 12 published cross sections. Boxed numbers are ratios of initial to final cross-sectional lengths; for example, a value of 2.63 corresponds to a cross section that is now $5.4 \mathrm{~km}$ long but is inferred to have been $14.1 \mathrm{~km}$ long, by unfolding one or more marker horizons. Data are listed in table 7. 
accurately placed at localities 12 and 13, where the Beck Pond and McKenney Ponds Limestones grade into or interfinger with the Seboomook Group and Tarratine Formation, respectively. Carbonate rocks of Lochkovian age include parts of the lower Fish River Lake Formation (loc. 21), Spider Lake Formation (loc. 18), and Square Lake Limestone (loc. 23). These rocks presumably were deposited beyond the limits of the Lochkovian foreland basin.

\section{Pragian}

Two lines of evidence show that the Pragian foreland basin may have migrated 15 to $20 \mathrm{~km}$ (present distance) cratonward from its early Lochkovian position (fig. 11). Along the Munsungun anticlinorium (loc. 16, fig. 1), the Pragian Seboomook flysch encroached over Lochkovian far-foreland carbonates. Similarly, in the Pennington anticlinorium (loc. 23), Pragian and younger flyschlike siltstone of unit Dus overlies the Square Lake Limestone. Within the foreland basin itself, the deltaic Matagamon Sandstone prograded over prodelta turbidites of the Seboomook Group. Although we have no direct evidence for migration of the deformation front from its Lochkovian position, in constructing figure 10 , we have assumed that the deformational front migrated the same distance as the foreland-basin margin did from its previous position (fig. 11). This assumption seems reasonable because the deformation front and foreland-basin front did migrate in tandem during every interval, such as Ludlow to Lochkovian, for which have adequate data.

\section{Early Emsian}

The clearest picture of Acadian paleogeography in Maine is in the early Emsian, at 407 to $406 \mathrm{Ma}$ (Bradley and others, 1996). We can identify a foreland basin and orogenic wedge. Superimposed on both basin and wedge and straddling the deformation front was a volcanic-plutonic belt. Many Emsian plutons truncate Acadian map-scale structures, including the newly dated Sebec Lake, Russell Mountain, and ShirleyBlanchard (SL, RU, and SB, respectively, fig. 6). The deformation front was plotted to the northwest of such plutons. The early Emsian foreland basin includes the younger strata of the vast "Devonian slate belt" of Boucot (1970). Forelandbasin flysch units that have yielded Emsian fossils include the Temiscouata Formation (loc. 24, fig. 1), the Compton Formation (locs. 3, 4), and the Littleton Formation (locs. 9, 10). It seems likely, though unproved, that parts of the Seboomook Group in the Connecticut Valley-Gaspé Basin in northwestern Maine and the Gile Mountain Formation of Vermont are also Emsian. Early Emsian molasse deposits in the study area (fig. 1) include the Tomhegan Formation (loc. 15) and, as suggested above, the uppermost part of the Matagamon Sandstone (loc. 17). These molasse facies must have been laid down near the proximal, southeastern margin of the foreland basin. The cratonward boundary of the early Emsian foreland basin can be positioned by using negative evidence. As dis- cussed below, the three occurrences of Eifelian limestone along the northwestern margin of the Connecticut ValleyGaspé Basin rest everywhere on pre-Emsian rocks, and so Emsian foreland-basin deposits- which are kilometers thick where present-probably were never deposited that far northwest.

The deformation front must have corresponded to the Lobster Mountains anticlinorium (LMA, fig. 2), between the site of Tomhegan molasse deposition and the site of the plutons that were emplaced into the deforming or recently deformed orogenic wedge. The Moxie and Katahdin plutons (KA and MX, respectively, fig. 6) were emplaced in the general vicinity of the deformation front (Bradley and others, 1996).

\section{Late Emsian to Early Eifelian}

By the time of the Emsian-Eifelian boundary ( 394 Ma), the Acadian deformation front had migrated some distance cratonward, to the approximate position of the Pennington anticlinorium (PA, fig. 2). Southeast of the deformation front (fig. 10), late Emsian and younger strata postdate Acadian deformation. To the northwest, foreland-basin and far-foreland deposits of this age range predate Acadian deformation. At locality 22 (fig. 1), the upper siliciclastic part of Boone's (1970) Fish River Lake Formation ("Nadeau Thoroughfare beds") were deposited in a foreland-basin setting. At localities 2, 5, and 7, Eifelian limestone was deposited in a far-foreland setting. The Mapleton Formation and Trout Valley Formation are similar in age to the upper part of the Fish River Lake Formation. Both the Mapleton and Trout Valley Formations, however, postdate the main phase of Acadian deformation, and so we place them southeast of the deformation front.

The stratigraphy of the Carlisle area (loc. 33, fig. 1) complicates an otherwise-straightforward paleogeographic picture. Though coeval with the Mapleton Formation, the Wapske Formation was involved in the same regional "Acadian" folding as the rest of the Silurian to Devonian section. One possible explanation is that the Carlisle area escaped Acadian deformation until the late Eifelian or later (figs. 10, 12). A second possible explanation is that the first phase of Acadian deformation, which in the Presque Isle area created the unconformity below the Mapleton Formation, bypassed the Carlisle area entirely. In this scenario, the folding at Carlisle would be related to the same, later event that caused gentle, late-stage or postAcadian folding of the Mapleton Formation at Presque Isle. A third possible explanation is that the Wapske Formation was deposited farther to the northwest and was thrust into its present position during a late-stage Acadian event.

\section{Late Eifelian to Early Givetian}

By the time of the Eifelian-Givetian boundary ( 387.5 Ma), the Acadian deformation front had again migrated cratonward from its previous position, especially on the basis of a belt of plutons in the Eastern Townships of Québec. These plutons intrude already-deformed Silurian and Devonian strata of the 
Connecticut Valley-Gaspé Basin; the oldest and, thus, most significant pluton is the Scotstown pluton (384 \pm 2 Ma; SC, fig. 6; Simonetti and Doig, 1990). The deformation front must have been situated somewhere to the northwest by about $384 \mathrm{Ma}$.

Just a few million years before emplacement of the Scotstown pluton, Eifelian carbonates were still being deposited at locality 5 (fig. 1). These strata are penetratively deformed, indicating that the final cratonward limit of Acadian deformation front was at least that far northwest. (The Paleozoic deformation front shown on figure 1 could be as old as Ordovician.) Exactly when the deformation took place is unknown, but it was likely at least a few million years after deposition of the last carbonates (the youngest being the Famine Limestone, loc. 5), long enough to allow for an interval of foreland-basin sedimentation before deformation.

The late Eifelian to early Givetian stratigraphic record in the study area (fig. 1) is confined to a few clasts in the Ste. Hélène Island breccia, near Montreal (loc. 1). The clasts include calcareous siltstone, sandstone, and some limestone of late Eifelian to early Givetian age (Boucot and others, 1986). By comparison of these rock types with the Catskill clastic wedge in New York, Montreal was probably near the cratonward edge of the Acadian foreland basin at about the Eifelian-Givetian boundary (fig. 11).

\section{Earliest Frasnian}

The deformation front at the beginning of Frasnian time has been plotted in figure 10 on the basis of slender evidence. Deformed Eifelian carbonates at localities 2, 5, and 7 (fig. 1) show that the Acadian orogeny was felt at least that far to the northwest. The deformation front was extrapolated from its position at the beginning of the Givetian, assuming a nearly constant convergence rate. The final northwestward limit of Acadian shortening is unknown; it might correspond to the Paleozoic thrust front (fig. 10) or, alternatively, might lie somewhere between the Frasian deformation front and the Paleozoic thrust front. Along the Paleozoic deformation front near Albany, N.Y., strata as young as Eifelian were involved in thrust faulting, probably Acadian but conceivably Alleghanian.

\section{Implications}

\section{Age of the Acadian orogeny}

A zone of active deformation migrated across Maine and adjacent parts of New England and Canada from early Ludlow through Givetian time. Initially, the orogen was a narrow belt, but it widened over time, eventually encompassing the entire State of Maine (fig. 10). The overall pattern of cratonward migration of the orogen is similar to that suggested by Donahoe and Pajari (1973) but is much changed in light of our new data from Maine and our improved time scale.

The Acadian orogeny has traditionally been regarded as a Devonian event. During the 1990's, however, new pluton ages were interpreted to show that the supposedly Devonian orogeny in parts of the northern Appalachians was actually Silurian (Bevier and Whalen, 1990b; West and others, 1992, 1995; Stewart and others, 1995a). Now, in light of revisions to the time scale that fix the Silurian-Devonian boundary at about $418 \mathrm{Ma}$, some of the purportedly Silurian plutons are back once again in the Early Devonian (for example, the North Pole Stream and Mount Elizabeth plutons of Bevier and Whalen, 1990b). The rest fall within the final 5 m.y. of the Silurian.

The northwestward migration of the deformation front (fig. 10) might be interpreted in two ways. One interpretation is that the orogeny occurred in pulses, each pulse separated by tectonic stability and each with some different cause. Some workers (for example, Boucot and others, 1964; Stewart and others, 1995a) have applied the term "Salinic" to Late Silurian orogenic events in Maine-events that, in their view, are distinct from the Devonian Acadian orogeny. Similarly, other names for orogenies might be coined for other positions of the deformation front (fig. 10). We prefer to explain the pattern in figure 10 as the result of a single plate-convergent event which swept the region over a long period of time. Existing data cannot resolve this question.

\section{Rate and Trajectory of Deformation-Front Migration}

In figure 10, the deformation front is shown to have migrated about $240 \mathrm{~km}$ across strike in about $40.5 \mathrm{~m}$.y. Assuming continuous rather than episodic convergence, this implies a rate of about $6 \mathrm{~km} / \mathrm{m}$.y. $(0.6 \mathrm{~cm} / \mathrm{yr})$. The actual rate must have been considerably faster because, as noted above, the base map is nonpalinspastic. Assuming, for the sake of discussion, that Acadian shortening reduced Maine to half its original across-strike width, the rate of thrust-front migration would be about $12 \mathrm{~km} / \mathrm{m} . \mathrm{y}$. (1.2 cm/yr).

It is likely, but not assured, that the rate of thrust-front migration approximately equaled the rate of plate convergence (Bradley and Kusky, 1986; Bradley, 1989). This is generally the case at oceanic subduction zones. Similarly, in the snowplow analogy for orogenic wedges (wherein a snowplow pushes a wedge of snow ahead of the plowblade), the deformation front in the snow migrates at about the same rate as the plow advances. A less likely alternative is that the advance of Acadian thrusts was directly linked not to plate convergence but to extensional orogenic collapse, as in the Carpathians (Royden and Burchfield, 1989). Although little is yet known about Acadian hinterland tectonics between about 420 and $380 \mathrm{Ma}$ (see section below entitled "Hinterland Deformational Regimes..."), there is no evidence for wholesale regional extension of the magnitude required by this mechanism.

On the basis of the along-strike diachronism of forelandbasin deposits in the northern Appalachians (younger toward the southwest), Bradley $(1987,1997)$ suggested that Acadian plate convergence had a dextral component. The migration of black-shale depocenters across the Acadian foreland basin in 
the central Appalachians led Ettensohn (1987) to the same conclusion. The present study area does not extend far enough along strike to provide clear evidence either for or against along-strike diachronism.

\section{Physical Setting of Magmatism}

One by-product of this study has been to show that many of the Acadian plutons of Maine occur in distinct age belts (fig. 6). Moreover, figure 12 shows how the various plutons and volcanic rocks were spatially related to the orogen as it existed at the time of magmatism.

\section{Coastal Volcanic Belt and the Ludlow to Lochkovian Plutonic Belt}

On geochemical grounds, Gates and Moench (1981), Hogan and Sinha (1989), and Seamans and others (1999) have suggested that the Ludlow to Lochkovian volcanic rocks and plutons of coastal Maine formed in an extensional setting. The migration pattern of the deformation front (figs. 10, 12), however, implies that magmatism took place in an overall setting of plate convergence. The same conclusion is suggested on a more local scale by the fact that the Lochkovian Lincoln sill was emplaced synkinematically (Tucker and others, in press). Judging from figure 12, magmatism took place in the upper plate of a two-plate system, presumably above an A-type subduction zone.

\section{Piscataquis Volcanic Belt and the Emsian Plutonic Belt}

The cause or causes of Ludlow to Emsian volcanism along the Piscataquis volcanic belt have long been debated, and although a resolution is beyond the scope of this report, our findings do provide an important new constraint: all of the volcanic rocks are broadly syncollisional and were erupted in a foreland setting. Most of the Ludlow to Lochkovian volcanic rocks (East Branch Group, West Branch Volcanics, and Fish River Lake, Spider Lake, Grand Lake Seboeis, and Allagash Lake Formations) were erupted in a far-foreland position. However, several volcanic units of Lochkovian and Emsian age are interbedded with or grade laterally into foreland-basin deposits and thus formed in a proximal-foreland position (Hedgehog Volcanics, Edmunds Hill Andesite, Hartin Formation, Traveler Rhyolite, and Kineo Rhyolite). Three alternative interpretations have been advanced for part or all of the Piscataquis volcanic belt. (1) On the basis of basalt discriminant plots, Hon and others (1992) and Keppie and Dostal (1994) interpreted the Spider Lake and West Branch volcanic rocks as having formed in an intracontinental-rift setting, related to so-called "transpressive rifting." In light of our findings, any extension would necessarily have taken place in a collisional foreland setting, such as might have been caused by lithospheric flexure (Bradley and Kidd, 1991) or by Baikal-style cracking of the lower plate (Sengör and others, 1978). We note that the geochemical signature of volcanic rocks in foreland settings has not been adequately characterized and that discriminant plots used by the above-cited authors do not even identify fields for volcanic rocks formed in this tectonic setting. (2) Tucker and others (in press) have suggested that the Emsian foreland-basin rhyolites and the Emsian plutons just to the southeast were a product of lower-lithospheric delamination of the downgoing (North American) plate, which was being subducted toward the southeast. (3) Bradley (1983) interpreted these units as arc volcanic rocks formed over a northwest-dipping subduction zone. This model would explain magmatism in all three settings - far foreland, proximal foreland, and orogenic wedge - as the consequence of an orogenic wedge above a southeast-dipping subduction zone colliding with (and overriding) a magmatic arc formed over a northwest-dipping subduction zone. (An updated version of this tectonic model was illustrated by Hanson and Bradley, 1989.)

\section{Syncollisional Versus Precollisional Plate Geometry}

Tectonic interpretations of the Acadian orogeny have long been debated. Part of the controversy regarding Acadian plate geometry may be sidestepped for present purposes by separately considering the plate geometry during two intervalsbefore initial impact of the converging plates and during the actual collision. The paired migration of the orogen and foreland basin shown in figures 10 through 12 suggests that, during collision, an upper plate consisting of the growing orogenic wedge and its Avalonian backstop overrode the Taconicmodified margin of North America. The implied A-type subduction zone that operated during collision presumably evolved naturally out of a B-type subduction zone of the same southeast-dipping polarity, where ocean floor that once existed between Avalonia and North America was consumed during the late Llandovery and Wenlock.

What remains to be settled is the nature of the northwestern margin of the Central Maine Basin before collision. This is a much-debated topic, and we, the coauthors, are ourselves divided on the subject. In the two main tectonic models, both of which have variants, it was either (1) a "passive" or rifted margin on the backside of an arc that had collided with North America during Ordovician time (for example, Robinson and others, 1998; Tucker and others, in press), or (2) a convergent margin that took up some of the motion between Avalonia and North America during Silurian time (Bradley, 1983; Eusden and others, 1996). In the second model, the northwestern subduction complex and arc were overridden by, and downflexed beneath, the southeastern one described in the preceding paragraph (Bradley, 1983). In the one-subduction-zone model (Robinson and others, 1998), the northwestern passive margin was overridden by, and downflexed beneath, a southeastern subduction-collision complex. Although the two models differ significantly up until the time of collision, they are much the same afterward. 


\section{Hinterland Deformational Regimes and Regional Partitioning of Acadian Deformation}

The present study has focused on tracking the first Acadian contractional deformation. In most places, however, Acadian deformation was polyphase. Figure 10 provides a framework for understanding those post-D1 events that occurred from about 423 to $382.5 \mathrm{Ma}$ - that is, the ages of the oldest and youngest deformation fronts. Three examples will be discussed here.

Stewart and others (1995a) described a complex history of motion on a network of northeast-striking dextral faults in Penobscot Bay. The oldest of these, the Penobscot BaySmith Cove-North Blue Hill Fault, displaces isograds around the Segwick pluton $(419.5 \pm 1 \mathrm{Ma})$ but is cut by the South Penobscot Intrusive Suite (419.2 $\pm 2.2 \mathrm{Ma})$. Later, the South Penobscot Intrusive Suite was cut by the dextral Turtle Head Fault, which was intruded, in turn, by the Lucerne pluton $(380 \pm 4$ Ma; Wones, 1991). Where plate convergence has an oblique component, deformation can be partitioned into thrusting in the orogenic wedge and strike-slip faulting farther to the rear (Dewey, 1980). If this explanation is applicable to the latest Silurian episode of dextral strike-slip faulting on the Maine coast, subduction of Central Maine Basin crust beneath the Coastal volcanic belt would have had a dextral-oblique component.

Near Waterville (WA, fig. 6), a 399-Ma intermediate dike cuts the first-phase Acadian folds but is deformed by a second generation of folds that must be older than a static metamorphic event dated at $380 \mathrm{Ma}$ (Osberg, 1988; Tucker and others, in press). The second folding must therefore have taken place when the Acadian deformation front was somewhere cratonward of its early Emsian position (fig. 10), perhaps $100 \mathrm{~km}$ to the northwest of Waterville. Accordingly, this contractional deformation was an out-of-sequence, hinterland event.

The Horserace pluton is a small body with a U-Pb age of $392 \mathrm{Ma}$ (R.D. Tucker, unpub. data) within the 407-Ma Katahdin batholith. It was emplaced, probably synkinematically (Hon, 1980, p. 73), along a northwest-striking highangle fault (the West Branch fault) that sinistrally offsets the western margin of the Katahdin pluton. This is one of many crossfaults that disrupt the bedrock map pattern in northern and central Maine (Osberg and others, 1985). Referring to figures 10 through 12, at about $392 \mathrm{Ma}$, the deformation front lay perhaps $70 \mathrm{~km}$ to the northwest. Like the second folding at Waterville, sinistral motion on the West Branch Fault took place in the Acadian hinterland, while the orogen was still advancing toward North America.

\section{Foreland Deformational Regimes}

Figures 10 through 12 also provide insights into preAcadian events that took place in what was then the orogenic foreland. Two northeast-striking high-angle faults at Spencer Mountain (loc. 12, fig. 1) cut the Hardwood Mountain Formation (Burroughs, 1979), to which we assign a late Ludlow to Pridoli age (table 2). The map pattern indicates that one of these faults is overlain by the Hobbstown Conglomerate, which ranges from possibly as old as Pridoli to definitely as young as Pragian (Boucot and Heath, 1969). The sense of motion is not well established. On the basis of the relative age of faulted strata, Burroughs (1979) showed both faults as having down-to-the-northwest motion. Whatever the exact age of faulting and the sense of motion, it is clear that faulting took place in a foreland setting and that the high-angle faults paralleled the approaching orogenic front. On this basis, they would appear to resemble normal faults of the Taconic foreland of New York, which formed in response to lithospheric flexure (Bradley and Kidd, 1991).

\section{Suggestions for Further Work}

Since an influential paper by Naylor (1970), the Acadian has commonly been described as "an abrupt and brief event." This description appears to be only half-true. Although the first phase of deformation was, indeed, abrupt, lasting but a few million years at any given place, it took some 40 m.y. for the deformation front to make its way across Maine. Our study thus confirms the findings of Donahoe and Pajari (1973), in general if not in particular.

Figures 10 through 12 could be improved with better age control for several key units. The Flume Ridge, Madrid, and Carrabassett Formations each have a particular tectonic significance but are so loosely dated by fossils as to be barely useful for the present purposes. A dedicated search for fossils, especially palynomorphs, and (or) datable pyroclastic horizons in these units would be worth the effort; tuff horizons, 1 to $2 \mathrm{~cm}$ thick, commonly yield zircons, and many of these horizons have probably been overlooked. Tighter paleontologic control is also needed, though less urgently, for the Hartin, Perry, and Swanback Formations, the Chapman Sandstone, and the Seboomook Group in northernmost Maine. Likewise, modern geochronologic studies are needed for the West Branch, Spider Lake, and Hedgehog Volcanics, the Red Beach and Lucerne plutons in Maine, and the many Devonian plutons in the Northeast Kingdom of Vermont.

The Devonian time scale still has significant holes to be filled in the Pragian, late Emsian, and Givetian; until these intervals are better calibrated, correlations between isotopically and paleontologically dated rocks in these age ranges will remain equivocal.

The depositional environments, paleocurrents, and provenance of unnamed unit Dsus and the Sangerville, Smalls Falls, Hartin, Wapske, Fish River Lake, and Swanback Formations warrant detailed study; we have assigned these units to a foreland-basin setting on the basis of lithology and regional relations alone. The Wapske is of special interest because it was evidently deformed about 15 m.y. later than rocks along strike in Maine (fig. 10). Accordingly, the Wapske Formation may have been deposited in a "piggyback" basin, a suggestion that might be evaluated by using detailed sedimentologic studies.

Our assessments of the pretectonic, syntectonic, or posttectonic age of plutons are admittedly cursory, based as they are on anecdotal observations of a few thin sections and 
one or two traverses from country rock into pluton. Detailed investigations would be especially useful for those plutons, such as the Moxie and Katahdin, that appear to have been emplaced very close to the deformation front.

The present study has emphasized evidence bearing on the northwestward migration of the Acadian orogen, but we have barely touched on its migration to the southeast. Did it migrate in a stepwise fashion, or gradually? Although the sedimentary record from the outboard side of the orogen is comparatively sparse, the Eastport Formation of coastal Maine and New Brunswick may preserve a record of synorogenic sedimentation off the backside of the growing Acadian mountains.

A palinspastic base will be essential for discerning the geographic relations between correlative rocks at the time they were formed and for quantifying the rate of plate convergence during collision.

Finally, one of the most intriguing recent developments in geodynamics involves previously unappreciated links between climate and tectonics. Judging from studies of the Southern Alps of New Zealand (for example, Beaumont and others, 1992), synorogenic rainfall and consequent erosion can profoundly affect the evolution of the underlying deforming rocks. Hoffman and Grotzinger (1993) suggested that an asymmetric orographic climate can influence the metamorphic field gradient, the width of the thrust belt, the extent of footwall involvement in deformation, and the thickness and compositional maturity of the foreland-basin fill. Acadian synorogenic climate and its possible influences on structure, metamorphism, and stratigraphy have not been investigated, but there is good reason to do so. Early Devonian paleogeographic reconstructions imply that the Acadian mountains had an asymmetric orographic climate, with the west side wetter (Witzke and Heckel, 1988). Such a climatic asymmetry, if borne out by paleoclimatologic studies of Devonian strata in the study area, might be explored as a possible control on the migration of the main Acadian deformation front.

\section{References Cited}

Abbott, R.N., Jr., 1991, Relationships between intrusive rocks in the Robbinston 15' quadrangle, Maine: New England Intercollegiate Geological Conference Annual Meeting, 83d, Flushing, N.Y., 1991, p. 133-156.

Albee, A.L., and Boudette, E.L., 1972, Geology of the Attean quadrangle, Somerset County, Maine: U.S. Geological Survey Bulletin 1297, $110 \mathrm{p}$.

Aleinikoff, J.N., and Moench, R.H., 1987, U-Pb geochronology and $\mathrm{Pb}$ isotopic systematics of plutonic rocks in northern New Hampshire; ensimatic vs. ensialic sources [abs.]: Geological Society of America Abstracts with Programs, v. 19, no. 1, p. 1.

Beaumont, C., Fullsack, P., and Hamilton, J., 1992, Erosional control of active compressional orogens, in McClay, K.R., ed., Thrust tectonics: London, Chapman and Hall, p. 1-18.

Berdan, J., 1971, Silurian to Early Devonian ostracodes of European aspect from the Eastport quadrangle, Maine [abs.]: Geological Society of America Abstracts with Programs, v. 3, no. 1, p. 18.
Berry, H.N., IV, and Osberg, P.H., 1989, A stratigraphic synthesis of eastern Maine and western New Brunswick: Maine Geological Survey Studies in Maine Geology, v. 2, p. 1-32.

Bevier, M.L., and Whalen, J.B., 1990a, U-Pb geochronology of Silurian granites, Miramichi terrane, New Brunswick, in Radiogenic age and isotopic studies, report 3: Geological Survey of Canada Paper 89-2, p. 93-100.

1990b, Tectonic significance of Silurian magmatism in the Canadian Appalachians: Geology, v. 18, p. 411-414.

Billings, M.P., 1937, Regional metamorphism of the Littleton-Moosilauke area, New Hampshire: Geological Society of America Bulletin, v. 48, p. 463-566.

Boone, G.M., 1958, Potassic feldspar enrichment in magma; origin of syenite in Deboullie district, northern Maine: Geological Society of America Bulletin, v. 73, p. 1451-1476.

1970, The Fish River Lake Formation and its environments of deposition: Maine Geological Survey Bulletin 23, p. 27-41.

Boucot, A.J., 1970, Devonian slate problems in the Northern Appalachians: Maine Geological Survey Bulletin 23, p. 42-48.

Boucot, A.J., and Arndt, R., 1960, Fossils of the Littleton Formation (Lower Devonian) of New Hampshire: U.S. Geological Survey Professional Paper 334-B, p. 41-51.

Boucot, A.J., Brett, C.E., Oliver, W.A., Jr., and Blodgett, R.B., 1986, Devonian faunas of the Sainte-Helene Island breccia, Montreal, Quebec, Canada: Canadian Journal of Earth Sciences, v. 23, p. 2047-2056.

Boucot, A.J., and Drapeau, G., 1968, Siluro-Devonian rocks of Lake Memphremagog and their correlatives in the Eastern Townships: Québec Department of Natural Resources Special Paper 1, $44 \mathrm{p}$.

Boucot, A.J., Field, M.T., Fletcher, R., Forbes, W.H., Naylor, R.S., and Pavlides, L., 1964, Reconnaissance bedrock geology of the Presque Isle quadrangle, Maine: Maine Geological Survey Quadrangle Mapping Series, no. 2, 123 p.

Boucot, A.J., Harper, C., and Rhea, K., 1959, Geology of the Beck Pond area, Township 3, Range 5, Somerset County, Maine: Maine Geological Survey Special Geologic Studies, no. 1, 33 p.

Boucot, A.J., and Heath, E.W., 1969, Geology of the Moose River and Roach River synclinoria, northwestern Maine: Maine Geological Survey Bulletin 21, $117 \mathrm{p}$.

Boucot, A.J., and Rumble, D., III, 1980, Regionally metamorphosed (high sillimanite zone, granulite facies) Early Devonian brachiopods from the Littleton Formation of New Hampshire: Journal of Paleontology, v. 54, p, 188-195.

Boudette, E.L., Hatch, N.L., Jr., and Harwood, D.S., 1976, Reconnaissance geology of the upper St. John and Allagash River basins, Maine: U.S. Geological Survey Bulletin 1406, 37 p.

Bradley, D.C., 1983, Tectonics of the Acadian orogeny in New England and adjacent Canada: Journal of Geology, v. 91, p. 381-400. 1987, Tectonic controls of stratigraphy in the Acadian foreland basin [abs.]: Geological Society of America Abstracts with Programs, v. 19, p. 6.

1989, Taconic plate kinematics as revealed by foredeep stratigraphy: Tectonics, v. 8, p. 1037-1049.

1997, The Northern Appalachians, in van der Pluijm, B., and Marshak, S., eds., Earth structure-an introduction to structure and tectonics: New York, WCB/McGraw-Hill, p. 445-450.

Bradley, D.C., and Hanson, L.S., 1989, Turbidites and melanges of 
the Madrid Formation, central Maine, in Berry, Jr., A.W., ed., Guidebook for field trips in southern and west-central Maine: New England Intercollegiate Geological Conference Annual Meeting, 81st, p. 183-199.

Bradley, D.C., and Kidd, W.S.F., 1991, Flexural extension of the upper continental crust in collisional foredeeps: Geological Society of America Bulletin, v. 103, p. 1416-1438.

Bradley, D.C., and Kusky, T., 1986, Geologic evidence for the rate of plate convergence during the Taconic arc-continent collision: Journal of Geology, v. 94, p. 667-681.

Bradley, D.C., Tucker, R., and Lux, D., 1996, Emsian position of the Acadian deformation front in Maine [abs.]: Geological Society of America Abstracts with Programs, v. 28, no. 7, p. 500.

Bradley, D.C., Tucker, R., Lux, D., Harris, A., and McGregor, D.C., 1998, Late Silurian to Middle Devonian migration of the Acadian deformation front across Maine [abs.]: Geological Society of America Abstracts with Programs, v. 30, no. 1, p. 7. 1999, Migration of the Acadian orogen and foreland basin across the Northern Appalachians: U.S. Geological Survey Open-File Report 98-770, 79 p.

Burroughs, W.A., 1979, Bedrock geology of the Spencer Lake quadrangle, Maine: Maine Geological Survey Open-File 79-1, 12 p., scale 1:62,500.

Dalrymple, G.B., Alexander, E.C., Jr., Lanphere, M.A., and Kraker, G.P., 1981 , Irradiation of samples for ${ }^{40} \mathrm{Ar} /{ }^{39} \mathrm{Ar}$ dating using the Geological Survey TRIGA reactor: U.S. Geological Survey Professional Paper 1176, $55 \mathrm{p}$.

Denning, A.S., and Lux, D.R., $1989,{ }^{40} \mathrm{Ar} /{ }^{39} \mathrm{Ar}$ isotopic dating of the Horserace Quartz Diorite and its bearing on age relationships in the Katahdin Batholith-Traveler Rhyolite system: Maine Geological Survey Studies in Maine Geology, v. 4, p. 115-123.

Dewey, J.F., 1980, Episodicity, sequence, and style at convergent plate boundaries, in Strangway, D.W., ed., The continental crust and its mineral deposits: Geological Association of Canada Special Paper 20, p. 553-573.

Donahoe, H.V., Jr., and Pajari, G., 1973, The age of Acadian deformation in Maine-New Brunswick: Maritime Sediments, v. 9, p. 78-82.

Eckren, E.B., and Frischknecht, F.C., 1967, Geological-geophysical investigations of bedrock in the Island Falls quadrangle, Aroostook and Penobscot Counties, Maine: U.S. Geological Survey Professional Paper 527, $36 \mathrm{p}$.

Elbert, D.C., Harris, A.G., and Denkler, K.E., 1988, Earliest Devonian conodonts from marbles of the Fitch Formation, Bernardston nappe, north-central Massachusetts: American Journal of Science, v. 288, p. 684-700.

Espenshade, G.H., and Boudette, E.L., 1967, Geology and petrology of the Greenville quadrangle, Piscataquis and Somerset Counties, Maine: U.S. Geological Survey Bulletin 1241-F, 60 p.

Ettensohn, F. J., 1987, Rates of relative plate motion during the Acadian Orogeny based on the spatial distribution of black shales: Geology, v. 95, p. 572-582.

Eusden, J.D., Jr., Garesche, J.M., Johnson, A.H., Maconochie, J., Peters, S.P., O'Brien, J.B., and Widmann, B.L., 1996, Stratigraphy and ductile structure of the Presidential Range, New Hampshire: Tectonic implications for the Acadian orogeny: Geological Society of America Bulletin, v. 108, p. 417-436.

Eusden, J.D., Jr., Guzovski, C.A., Robinson, A.C., and Tucker, R.D., in press, Timing of the Acadian Orogeny in northern New Hampshire: Journal of Geology.

Fyffe, L.R., 1995, Fredericton Belt, in Middle Paleozoic rocks, chap. 4 of Williams, Harold, ed., Geology of the Appalachian-Caledonian orogen in Canada and Greenland (Geology of North America series, v. F-1): Boulder, Colo., Geological Society of America, p. 351-354.

Gabis, C., Hon, R., and Hanson, L., 1994, A geochemical investigation of Early Devonian intrusions in the Monson-Greenville area: New England Intercollegiate Geological Conference Annual Meeting, 86th, Salem, Mass., Guidebook to Field Trips, p. 239-257.

Gates, 0., and Moench, R.H., 1981, Bimodal Silurian and Lower Devonian volcanic rock assemblages in the Machias-Eastport area, Maine: U.S. Geological Survey Professional Paper 1184, 32 p.

Griffin, J.R., 1971, Bedrock geology of the Guilford quadrangle, Maine: Maine Geological Survey Open-File 71-5, scale 1:62,500.

Griscom, A., 1976, Bedrock geology of the Harrington Lake area, Maine: Cambridge, Mass., Harvard University, Ph.D. thesis, 373 p.

Hall, B.A., 1970, Stratigraphy of the southern end of the Munsungun anticlinorium, Maine: Maine Geological Survey Bulletin 22, 63 p.

Hall, B.A., Pollock, S.J., and Dolan, K.M., 1976, Lower Devonian Seboomook Formation and Matagamon Sandstone, northern Maine; a flysch basin-margin delta complex, in Page, L.R., ed., Contributions to the stratigraphy of New England: Geological Society of America Memoir 148, p. 57-63.

Hanson, L.S., and Bradley, D.C., 1989, Sedimentary facies and tectonic interpretation of the Lower Devonian Carrabassett Formation, north-central Maine: Maine Geological Survey Studies in Maine Geology, v. 2, p. 101-125.

1993, Late Silurian to Early Devonian paleogeography lof the Kearsarge-Central Maine Basin revealed by paleocurrents and sedimentary facies [abs.]: Geological Society of America Abstracts with Programs, v. 25, no. 6, p. A-360.

Harland, W.B., Armstrong, R.L., Cox, A.V., Craig, L.E., Smith, A.G., and Smith, D.G., 1990, A geologic time scale, 1989: Cambridge, U.K., Cambridge University Press, $265 \mathrm{p}$.

Harris, A.G., Hatch, N.L., Jr., and Dutro, J.T., Jr., 1983, Late Silurian conodonts update the metamorphosed Fitch Formation, Littleton, New Hampshire: American Journal of Science, v. 283, p. 722-738.

Heitzler, M.T., Lux, D.R., and Decker, E.R., 1988, The age and cooling history of the Chain of Ponds and Big Island plutons and the Spider Lake granite, west-central Maine and Québec: American Journal of Science, v. 288, p. 925-952.

Hesse, R., and Dalton, E., 1995, Turbidite channel/overbank deposition in a Lower Devonian orogenic shale basin, Fortin Group of Gaspé Peninsula, Northern Appalachians, Canada: Journal of Sedimentary Research, v. B65, p. 44-60.

Hibbard, J.P., 1993, Geology of the Grand Lake Seboeis region, northcentral Maine; 1992 field report: Augusta, Maine Geological Survey, $11 \mathrm{p}$.

1994, Kinematics of deformation in the northern and Newfoundland Appalachians: Journal of Geology, v. 102, p. 215-228.

Hoffman, P.F., and Grotzinger, J.P., 1993, Orographic precipitation, erosional unroofing, and tectonic style: Geology, v. 21, p. 195-198. 
Hogan, J.P., and Sinha, A.K., 1989, Compositional variation of plutonism in the coastal Maine magmatic province; mode of origin and tectonic setting: Maine Geological Survey Studies in Maine Geology, v. 4, p. 1-33.

Hon, R., 1980, Geology and petrology of igneous bodies within the Katahdin batholith, in Roy, D. C., and Naylor, R. S., eds., A guidebook to the geology of northeastern Maine and neighboring New Brunswick: New England Intercollegiate Geological Conference Annual Meeting, 72d, p. 65-79.

Hon, R., Fitzgerald, J., Sargeant, S., Schwartz, W., Dostal, J., and Keppie, J.D., 1992, Silurian-Early Devonian mafic volcanic rocks of the Piscataquis volcanic belt in northern Maine: Atlantic Geology, v. 28, p. 163-170.

Hopeck, J.T., 1991, Post-Caradocian stratigraphy of the AroostookMatapedia and Miramichi lithotectonic blocks, in Ludman, A., ed., Geology of the coastal lithotectonic block and neighboring terranes, eastern Maine and southern New Brunswick: New England Intercollegiate Geological Conference Annual Meeting, 83d, p. 157-168.

Hubacher, F.A., and Lux, D.R., 1987, Timing of Acadian deformation in northeastern Maine: Geology, v. 15, p. 80-83.

Hueber, F.M., Bothner, W.A., Hatch, N.L., Jr., Finney, S.C., and Aleinikoff, J.N., 1990, Devonian plants from southern Québec and northern New Hampshire and the age of the Connecticut Valley trough: American Journal of Science, v. 290, p. 360-395.

Jaffey, A.H., Flynn, K.F., Glendenin, L.E., Bentley, W.C., and Essling, A.M., 1971, Precision measurement of half-lives and specific activities of ${ }^{235} \mathrm{U}$ and ${ }^{238} \mathrm{U}$ : Physical Review C: Nuclear Physics, v. 4, p. 1889-1906.

Jurinski, J., 1990, Petrogenesis of the Moosehorn igneous complex, Maine: Blacksburg, Virginia Polytechnic Institute, M.S. thesis.

Kasper, A.E., Jr., Gensel, P.G., Forbes, W.H., and Andrews, H.N., Jr., 1988, Plant paleontology in the State of Maine - a review: Maine Geological Survey Studies in Maine Geology, v. 1, p. 109-128.

Keppie, J.D., and Dostal, J., 1994, Late Silurian-Early Devonian transpressive rift origin of the Québec Reentrant, northern Appalachians; constraints from geochemistry of volcanic rocks: Tectonics, v. 13, p. 1183-1189.

Krogh, T.E., 1973, A low-contamination method for hydrothermal decomposition of zircon and extraction of $\mathrm{U}$ and $\mathrm{Pb}$ for isotopic age determinations: Geochimica et Cosmochimica Acta, v. 37, p. 485-494.

-1982, Improved accuracy of U-Pb zircon dating by the creation of more concordant systems using air abrasion techniques: Geochimica et Cosmochimica Acta, v. 46, p. 637-649.

Ludman, A. 1978, Geologic map of the Kingsbury quadrangle: Maine Geological Survey Geologic Map Series, no. GM-6, 36 p., scale $1: 62,500$.

-1981, Significance of transcurrent faulting in eastern Maine and location of the suture between Avalonia and North America: American Journal of Science, v. 281, p. 463-483. 1990, Revised bedrock geology of the Danforth, Scraggly Lake, Forest, Waite, Vanceboro, and Kellyland 15' quadrangles, Maine: Maine Geological Survey Geologic Map Series, 36 p., scale 1:62,500.

Ludman, A., Hopeck, J.T., and Brock, P.C., 1993, Nature of the Acadian orogeny in eastern Maine: Geological Society of America Spe- cial Paper 275, p. 67-84.

Ludman, A., and Idleman, B., 1998, The Berry Brook gabbro; NW-most Siluro-Devonian mafic magmatism in the Coastal Lithotectonic Belt, Maine [abs.]: Geological Society of America Abstracts with Programs, v. 30, no. 1, p. 58.

Ludman, A., and West, D.P., Jr., 1999, The Norumbega fault system of the Northern Appalachians: Geological Society of America Special Paper 331, $202 \mathrm{p}$.

Ludwig, K.R., 1980, Calculation of uncertainties of U-Pb isotope data: Earth and Planetary Science Letters, v. 46, p. 212-220. 1992, ISOPLOT_a plotting and regression program for radiogenic-isotope data, version 2.57: U.S. Geological Survey OpenFile Report 91-445, $40 \mathrm{p}$.

Lux, D.R., and Aleinikoff, J.N., $1985,{ }^{40} \mathrm{Ar} /{ }^{39} \mathrm{Ar}$ geochronology of the Songo pluton, western Maine [abs.]: Geological Society of America Abstracts with Programs, v. 17, no. 1, p. 32.

Lux, D.R., Gibson, D., and Hamilton, P.J., 1989, Geochronology of the Songo pluton, western Maine: Maine Geological Survey Studies in Maine Geology, v. 4, p. 101-114.

Marvin, R.F., and Dobson, S.W., eds., Radiometric ages; compilation B, U.S. Geological Survey: Isochron/West, no. 26, p. 3-32.

Marvinney, R.G., 1984, The Forks Formation of northwestern Maine: Evidence for a Late Ordovician to Late Silurian angular unconformity: Northeastern Geology, v. 6, p. 151-160.

McGregor, D.C., 1962, Report to Dr. Ely Mencher (Massachusetts Institute of Technology) on spore analysis of samples from St. Froid Lake, Maine: Geological Survey of Canada Report F1-12-1962-DCM, 2 p.

1963, Report to Dr. Ely Mencher (Massachusetts Institute of Technology) on palynological analysis of samples from Aroostook County, Maine: Geological Survey of Canada Report F1-8-1963-DCM, 2 p.

1968, Report to Dr. Ely Mencher of the Massachusetts Institute of Technology on palynological analysis of samples from Maine, U.S.A: Geological Survey of Canada Report F1-121968-DCM, 2 p.

1992, Palynomorph evidence for the age of the Trout Valley Formation of northern Maine: Geological Survey of Canada Report F1-9-1992-DCM, 4 p.

1996, Palynology of outcrop samples of Devonian age from the Fish River Lake Formation, Maine, U.S.A.: Geological Survey of Canada Report F1-1-1996-DCM, 5 p.

McKerrow, W.S., and Ziegler, A.M., 1971, The Lower Silurian paleogeography of New Brunswick and adjacent areas: Journal of Geology, v. 79, p. 635-646.

Moench, R.H., 1971, Geologic map of the Rangeley and Phillips quadrangles, Franklin and Oxford Counties, Maine: U.S. Geological Survey Miscellaneous Investigations Series Map I-605, scale $1: 62,500$.

Moench, R.H., Boone, G.M., Bothner, W.A., Boudette, E.L., Hatch, N.L., Jr., Hussey, A.M., II, Marvinney, R.G., and Aleinikoff, J.N., 1995, Geologic map of the Sherbrook-Lewiston area, Maine, New Hampshire, and Vermont, United States, and Québec, Canada: U.S. Geological Survey Miscellaneous Investigations Series Map I-1898-D, 56 p., scale 1:250,000, 2 sheets.

Moench, R.H., and Pankiwskyj, K.A., 1988, Geologic map of western interior Maine: U.S. Geological Survey Miscellaneous Investigations Series Map I-1692, 22 p., scale 1:250,000. 
Naylor, R.S., 1970, The Acadian Orogeny; an abrupt and brief event: Science, v. 172, p. 558-560.

Neumann, R.B., 1967, Bedrock geology of the Shin Pond and Stacyville quadrangles, Penobscot County, Maine: U.S. Geological Survey Professional Paper 524-I, 37 p.

Oliver, W.A., Jr., 1971, The coral fauna of the Famine Limestone in Québec: Smithsonian Contributions to Paleontology, no. 3, p. 193-201.

Osberg, P.H., 1988, Geologic relations within the shale-wacke sequence in south-central Maine: Maine Geological Survey Studies in Maine Geology, v. 1, p. 51-73.

Osberg, P.H., Hussey, A.M., II, and Boone, G.M., 1985, Bedrock geologic map of Maine: Augusta, Maine Geological Survey, scale 1:500,000.

Osberg, P.H., Tull, J.F., Robinson, P., Hon, R., and Butler, J.R., 1989, The Acadian orogen: in Hatcher, R.D., Jr., Thomas, W.A., and Viele, G.W., eds., The Appalachian-Ouachita orogen in the United States (The Geology of North America, v. F-2): Boulder, Colo., Geological Society of America, p. 179-232.

Pankiwskyj, K.A., Ludman, A., Griffin, J.R., and Berry, W.B.N., 1976, Stratigraphic relationships on the southeast limb of the Merrimack synclinorium in central and west-central Maine, in Lyons, P.C., and Brownlow, A.H., eds., Studies in New England geology (C. Wroe Wolfe volume): Geological Society of America Memoir 146, p. 263-280.

Pavlides, L., 1962, Geology and manganese deposits of the Maple and Hovey Mountains area, Aroostook County, Maine: U.S. Geological Survey Professional Paper 362, 116 p.

Pickerill, R.K., and Pajari, G.E., Jr., 1976, The Eastport Formation (Lower Devonian) in the northern Passamaquoddy Bay area, southwest New Brunswick: Canadian Journal of Earth Sciences, v. 13, p. 266-270.

Pickerill, R.K., Pajari, G.E., and Dickson, W.L., 1978, Geology of the Lower Devonian rocks of Pasamaquoddy Bay, southwest New Brunswick, in Ludman, A., ed., Guidebook for field trips in southeastern Maine and Southwestern New Brunswick: New England Intercollegiate Geological Conference Annual Meeting, 70th, Flushing, N.Y., 1978, p. 38-56.

Pollock, S.G., 1985, Bedrock geology of the Caucomgomoc Lake area, Maine: Maine Geological Survey Open-File 85-85, scale $1: 62,500$.

1987, The Lower Devonian slate problem of western and northern Maine revisited: Northeastern Geology, v. 9, p. 37-50.

Pollock, S.G., Boucot, A. J., and Hall, B. A., 1988, Lower Devonian deltaic sedimentary environments and ecology; examples from the Matagamon Sandstone, northern Maine: Maine Geological Survey Studies in Maine Geology, v. 1, p. 81-99.

Rankin, D., 1968, Volcanism related to tectonism in the Piscataquis volcanic belt, an island arc of early Devonian age in north-central Maine, in Zen, E-an, White, W.S., Hadley, J.B., and Thompson, J.B., Jr., eds., Studies of Appalachian geology, northern and maritime: New York, Interscience, p. 355-369.

Rankin, D.W., and Tucker, R.D., 1995, U-Pb age of the Katahdin-Traveler igneous suite, Maine, local age of the Acadian orogeny, and thickness of the Taconian crust [abs.]: Geological Society of America Abstracts with Programs, v. 27, no. 6, p. A-225.

Richardson, J.B., and McGregor, D.C., 1986, Silurian and Devonian spore zones of the Old Red Sandstone continent and adjacent regions: Geological Survey of Canada Bulletin 364, 78 p.

Robinson, P., and Tucker, R., 1996, The "Acadian" in central New England; new problems based on $\mathrm{U}-\mathrm{Pb}$ ages of igneous zircon and metamorphic monazite and sphene [abs.]: Geological Society of America Abstracts with Programs, v. 28, no. 3, p. 94.

Robinson, P., Tucker, R.D., Bradley, D.C., Berry, H.N., IV, and Osberg, P.H., 1998, Paleozoic orogens in New England, U.S.A.: GFF (Geological Society of Sweden Quarterly Journal), v. 120, p. 119-148.

Roden, M.K., Parrish, R.R., and Miller, D.S., 1990, The absolute age of the Eifelian Tioga ash bed: Journal of Geology, v. 98, p. 282-285.

Roy, D. C. , 1980, Tectonics and sedimentation in northeastern Maine and adjacent New Brunswick, in Roy, D.C., and Naylor, R.S., eds., A guidebook to the geology of northeastern Maine and neighboring New Brunswick: New England Intercollegiate Geological Conference Annual Meeting, 72d, p. 1-21.

1989, The Depot Mountain Formation; transition from synto post-Taconian basin along the Baie Vert-Brompton Line in northwestern Maine: Maine Geological Survey Studies in Maine Geology, v. 2, p. 85-99.

Roy, D.C., and Mencher, E., 1976, Ordovician and Silurian stratigraphy of northeastern Aroostook County, Maine, in Page, L.R., ed., Contributions to the stratigraphy of New England: Geological Society of America Memoir 148, p. 25-52.

Roy, D.C., and White, K.J., 1987, The Mapleton Formation; a post-Acadian basin sequence, Presque Isle, Maine: Geological Society of America, Northeastern Section Centennial Field Guide, p. 311-316.

Royden, W., and Burchfiel, B.C., 1989, Are systematic variations in thrust belt style related to plate-boundary processes? (The western Alps versus the Carpathians): Tectonics, v. 8, p. 51-62.

St. Peter, C., 1977, Geology of parts of Restigouche, Victoria, and Madawaska Counties, northwestern New Brunswick, N.T.S. 21 N/8, 21 N/9, 21 0/5, 21 0/11, and 21 0/14: New Brunswick Department of Natural Resources, Geological Surveys Branch Report of Investigation 17, 69 p., scale 1:50,000, 5 sheets. 1982, Geology of the Jupiter-Knowlesville-Carlisle area, New Brunswick, Map-areas I-16, I-17, and I-18: New Brunswick Department of Natural Resources, Geological Surveys Branch Map Report 82-1, 82 p., scale 1:25,000, 3 sheets.

St. Peter, C.J., and Boucot, A.J., 1981, Age and regional significance of brachiopods from the Temiscouata Formation of Madawaska County, New Brunswick: Maritime Sediments and Atlantic Geology, v. 17, p. 88-95.

Scambos, T.A., 1980, Petrology and geochemistry of the Center Pond pluton, Lincoln, Maine: Blacksburg, Virginia Polytechnic Institute, M.S. thesis, $89 \mathrm{p}$.

Seaman, S.J., Wobus, R.A., Wiebe, R.A., Lubisk, N., and Bowring, S.A., 1995, Volcanic expression of bimodal magmatism; the Cranberry Island-Cadillac Mountain complex, coastal Maine: Journal of Geology, v. 103, p. 301-311.

Seaman, S.J., Scherer, E.E., Wobus, R.A., Zimmer, J.H., and Sales, J.G., 1999, Late Silurian volcanism in coastal Maine; the Cranberry Island series: Geological Society of America Bulletin, v. 111, p. 686-708.

Sengör, A.M.C., Burke, K., and Dewey, J.F., 1978, Rifts at high angles to orogenic belts; tests for their origin and the Upper Rhine graben 
as an example: American Journal of Science, v. 278, p. 24-40.

Simonetti, A., and Doig, R., 1990, U-Pb and Rb-Sr geochronology of Acadian plutonism in the Dunnage zone of the southeastern Québec Appalachians: Canadian Journal of Earth Sciences, v. 27, p. 881-892.

Slivitski, A., and St.-Julien, P., 1987, Compilation géologique de la région de l'Estrie-Beauce: Ministère de l'Énergie et des Ressources Québec, MM 8504, 40 p., scale 1:250,000.

Solar, G.S., Pressley, R., Brown, M. and Tucker, R.D., 1998, Granite ascent in convergent orogenic belts; testing a model: Geology, v. 26, p. 711-714.

Spooner, C.W., and Fairbairn, H.W., 1970, Relation of radiometric age of granitic rocks near Calais, Maine, to the time of Acadian orogeny: Geological Society of America Bulletin, v. 81, p. 3663-3670.

Stacey, J.S., and Kramers, J.D., 1975, Approximation of terrestrial lead isotope evolution by a two-stage model: Earth and Planetary Science Letters, v. 26, p. 207-221.

Steiger, R.H., and Jäger, E., 1977, Subcommission on Geochronology; convention on decay constants in geo- and cosmochronology: Earth and Planetary Science Letters, v. 36, p. 359-363.

Stewart, D.B., Tucker, R.D., and West, D.P., Jr., 1995b, Genesis of Silurian composite terrane in northern Penobscot Bay region, Maine, in Hussey, A.M., Jr., and Johnson, R.A., eds., Guidebook to field trips in southern Maine and adjacent New Hampshire: New England Intercollegiate Geological Conference Annual Meeting, 87th, Brunswick, Maine, 1995, p. A3-1 to A3-21.

Stewart, D.B., Unger, J.D., and Hutchinson, D.R., 1995a, Silurian tectonic history of Penobscot Bay region, Maine: Atlantic Geology, v. 31, p. 67-69.

Thompson, J.B., Jr., Robinson, P., Clifford, T.N., and Trast, N., 1968, Nappes and gneiss domes in west-central New England, in Zen, E-an, White, W.S., Hadley, J.B., and Thompson, J.B., Jr., eds., Studies of Appalachian geology, northern and maritime: New York, Interscience, p. 203-218.

Tucker, R.D., Bradley, D.C., ver Straeten, C.A., Harris, A.G., Ebert, J.R., and McCutcheon, S.R., 1998, New U-Pb zircon ages and the duration and division of Devonian time: Earth and Planetary Science Letters, v. 158, no. 3-4, p. 175-186.

Tucker, R.D., and McKerrow, W.S., 1995, Early Paleozoic chronology; a review in light of new $\mathrm{U}-\mathrm{Pb}$ zircon ages from Newfoundland and
Britain: Canadian Journal of Earth Sciences, v. 32, p. 368-379.

Tucker, R.D., Osberg, P.H., and Berry, H.N., IV, in press, The geology of a part of Acadia and the nature of the Acadian orogeny across central and eastern Maine: American Journal of Science.

Tucker, R.D., and Robinson, P., 1990, Age and setting of the Bronson Hill magmatic arc; a re-evaluation based on $\mathrm{U}-\mathrm{Pb}$ zircon ages in southern New England: Geological Society of America Bulletin, v. 102, p. 1404-1419.

Uyeno, T.T., and Lespérance, P.J., 1997, Middle Devonian (Eifelian) conodonts, and their stratigraphic implications, from the midAppalachians of Québec, in Klapper, G., Murphy, M.A., and Talent, J.A., eds., Paleozoic sequence stratigraphy, biostratigraphy, and biogeography; studies in honor of J. Grenville ("Jess") Johnson: Geological Society of America Special Paper 321, p. 145-160.

Van Heteren, J., and Kusky, T., 1994, Posttectonic intrusion of the Onawa pluton, central Maine [abs.]: Geological Society of America Abstracts with Programs, v. 26, no. 3, p. 77.

Venugopal, D.V., 1979, Geology of the Debec Junction-Gibson-Millstream-Temperance Vale-Meductic region, Map-areas G-21, $\mathrm{H}-21, \mathrm{I}-21$, and H-22: New Brunswick Department of Natural Resources, Geological Surveys Branch Map Report 79-5, 36 p., scale $1: 25,000,4$ sheets.

West, D.P., Guidotti, C.V., and Lux, D.R., 1995, Silurian orogenesis in western Penobscot Bay region, Maine: Canadian Journal of Earth Sciences, v. 32, p. 1845-1858.

West, D.P., Ludman, A., and Lux, D.R., 1992, Silurian age for the Pocomoonshine gabbro-diorite, southeastern Maine and its regional tectonic implications: American Journal of Science, v. 292, p. 253-273.

Wilson, J.T., 1966, Did the Atlantic close and then re-open again?: Nature, v. 211, p. 676-686.

Witzke, B.J., and Heckel, P.H., 1988, Paleoclimatic indicators and inferred Devonian paleolatitudes of Euramerica, in McMillan, N.J., Embry, A.F., and Glass, D.J., eds., Devonian of the world: International Symposium on the Devonian System, 2d, Proceedings, v. 1, p. 49-63.

Wones, D.R., 1991, Bedrock geologic map of the Orland quadrangle, Hancock and Penobscot Counties, Maine: U.S. Geological Survey Geologic Quadrangle Map GQ-1692, scale 1:62,500. 



\section{PLATES 1 AND 2}

[Contact photographs of the plates in this report are available, at cost, from the U.S. Geological Survey Library, Federal Center, Denver, Colorado 80225] 
Plate 1.-Late Silurian to early Middle Devonian (late Ludlow to Eifelian) conodonts from Maine and southern Quebec, Canada. Scanning-electron photomicrographs. Illustrated specimens are reposited in the U.S. National Museum (USNM), Washington, D.C. See text figure 1 for geographic location (locality no.), text figure 4 for stratigraphic position, and table 2 for faunal assemblage, age assignment, biofacies, and conodont-alteration index.

Figures 1-13. Beck Pond Limestone, locality 12. USGS colln. 12518-SD. Magnification, 50×.

1-6. Ozarkodina remscheidensis eosteinhornensis (Walliser), Pa, inner (figs. 1, 3) and outer (fig. 2) lateral views, $\mathrm{Pb}, \mathrm{M}$, and Sc (lateral views) elements. USNM nos. 508893-508897.

7, 8. $\quad$ Dvorakia sp., Sb element, posterior and anterior views. USNM no. 508898.

9, 10. Icriodus sp. indet., coniform Sc element, outer and inner lateral views. USNM no. 508899.

11, 12. Decoriconus fragilis (Branson and Mehl), inner and outer lateral views. USNM no. 508900.

13. Belodella sp., Sa element, lateral view. USNM no. 508901.

14-18. McKenney Ponds Limestone, locality 13. USGS colln. 12515-SD.

14, 15. Pseudooneotodus beckmanni (Bischoff and Sannemann), upper and lateral views. Magnification, 75×. USNM no. 508902.

16-18. Icriodus sp. indet., I elements (incomplete), upper views. Magnifications, 40× (figs. 16, 17) and 35× (fig. 18). USNM nos. 508903-508905.

19-26. McKenney Ponds Limestone, locality 13. USGS colln. 12516-SD.

19-24. Icriodus sp. indet., coniform elements. Magnification, 40×.

19, 20. Sa element, opposite lateral views. USNM no. 508906.

21, 22. M? element, inner and outer lateral views. USNM no. 508907.

23, 24. Sd element, opposite lateral views, USNM no. 508908.

25, 26. Ozarkodina remscheidensis (Ziegler), Pa elements, inner lateral views. Magnification, 50×. USNM nos. 508909-508910.

27, 28. Ozarkodina remscheidensis (Ziegler), Pa elements, inner lateral and upper views. Magnification, 50×. USNM nos. 508911-508912, McKenney Ponds Limestone. USGS colln. 12517-SD.

29-31. Mountain House Wharf Limestone, locality 2. Icriodus sp. indet., I elements, upper views. Magnification, 45×.

29. USNM no. 508913, USGS colln. 12527-SD.

30, 31. USNM nos. 508914-508915, USGS colln. 12526-SD. 


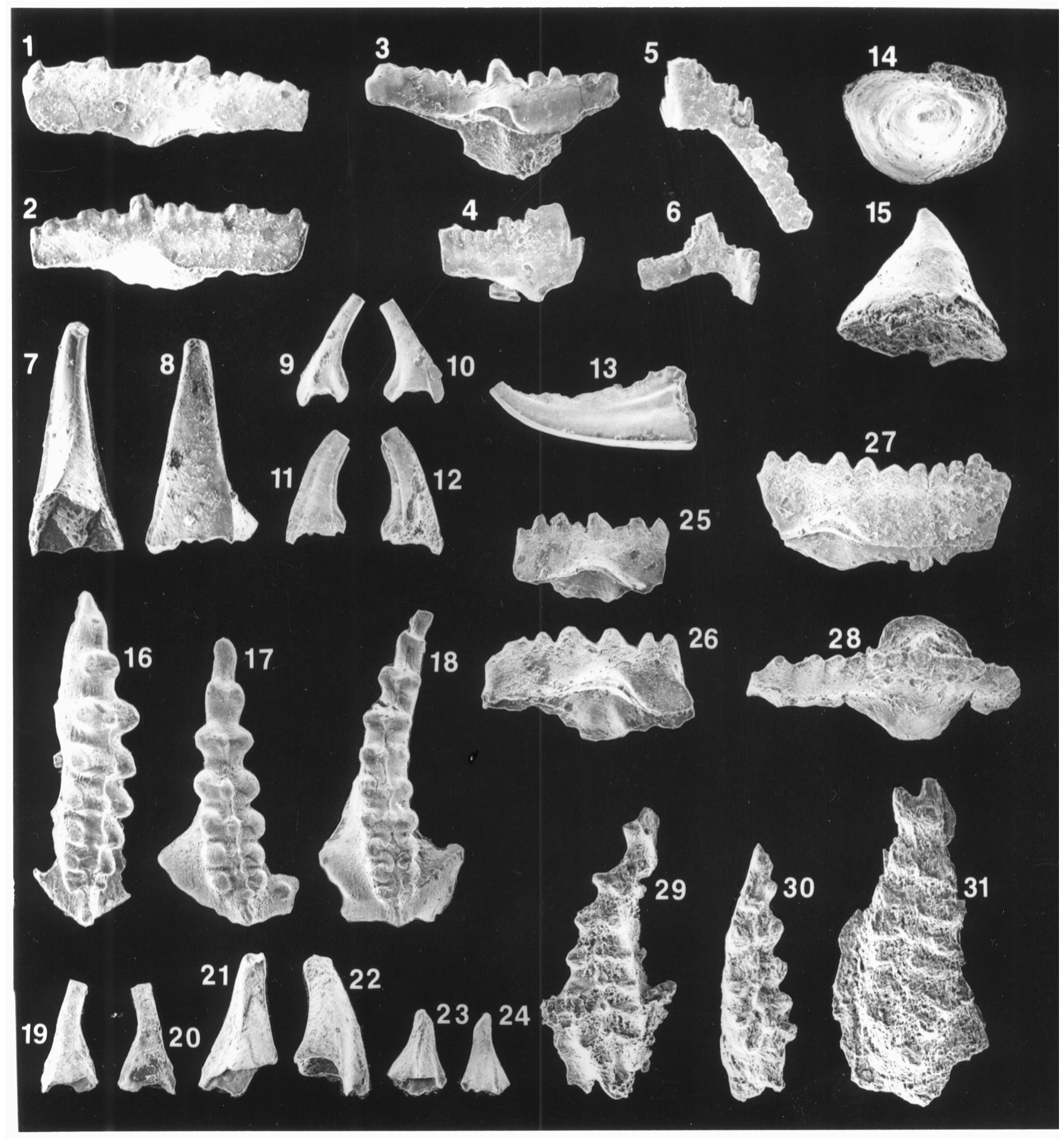


Plate 2.- Late Silurian to earliest Devonian (late Ludlow to early Lochkovian) conodonts from Maine. Scanning-electron photomicrographs; illustrated specimens are reposited in the U.S. National Museum (USNM), Washington, D.C. See text figure 1 for geographic location (locality no.), text figure 4 for stratigraphic position, and table 2 for faunal assemblage, age assignment, biofacies, and conodont-alteration index.

Figures 1-5. Ripogenus Formation, locality 16. USGS colln. 12513-SD.

1, 2. Dvorakia sp. indet., inner and outer lateral views of Sb element. Magnification, 50×. USNM no. 508869.

3, 4. Ozarkodina excavata (Branson and Mehl), inner lateral views of Sb and Sc elements. Magnification, 50×. USNM nos. 508870-508871.

5. Digyrate apparatus, inner lateral view of Sb element. Magnification, 60×. USNM no. 508872.

6-9. Parker Bog Formation, locality 14. Oulodus elegans, inner lateral views of $\mathrm{Pa}, \mathrm{Pb}, \mathrm{M}$, and Sb elements. Magnification, 60×. USNM nos. 508873-508876, USGS colln. 12521-SD.

10, 11. Parker Bog Formation, locality 14. Pedavis sp. indet., posterior and anterior views of coniform S element. Magnification, 75×. USNM no. 508877, USGS colln. 12521-SD.

12-20. Hardwood Mountain Formation, locality 11. USGS colln. 12523-SD.

12. Ozarkodina excavata excavata (Branson and Mehl), Pa element, outer lateral view. Magnification, 50×. USNM no. 508878.

13-16. Corryssognathus dubius (Rhodes), Sb and Sc elements, outer and inner lateral and posterior and anterior views. Magnification, 50×. USNM nos. 508879-508880.

17-20. Ozarkodina remscheidensis remscheidensis (Ziegler), Pa elements, inner and outer lateral views. Magnifications, 45× (figs. 17, 18) and 50× (figs. 19, 20). USNM nos. 508881-508882.

21. Hardwood Mountain Formation, locality 11. Ozarkodina confluens Branson and Mehl, Pa element, inner lateral view. Magnification, 35×. USNM no. 508883, USGS colln. 12524-SD.

22-38. Square Lake Limestone, locality 23. USGS colln. 12511-SD.

22-26. Decoriconus fragilis (Branson and Mehl). Magnification, 75×.

22, 23. Sc elements (23 lost after photography), inner lateral views, USNM no. 508884.

24-26. Sb elements, inner and two outer lateral views of two specimens, USNM nos. 508885 and 508886.

27-35. Belodella cf. B. resima (Philip). Magnifications, $60 \times$ (figs. 27-34) and 110× (fig. 35).

27, 28. M element, outer and inner lateral views, USNM no. 508887.

29, 30, 35 Sb1 element, inner and outer lateral and posterior views. USNM no. 508888.

31, 32. Sa element, opposite lateral views. USNM no. 508889.

33, 34. Sc element, outer and inner lateral views. USNM no. 508890.

36, 37. Dvorakia sp., Sc element, inner and outer lateral views. Magnification, 50×. USNM no. 508891.

38. Pseudooneotodus beckmanni (Bischoff and Sannemann), oblique lower posterior view. Magnification, 75×. USNM no. 508892. 


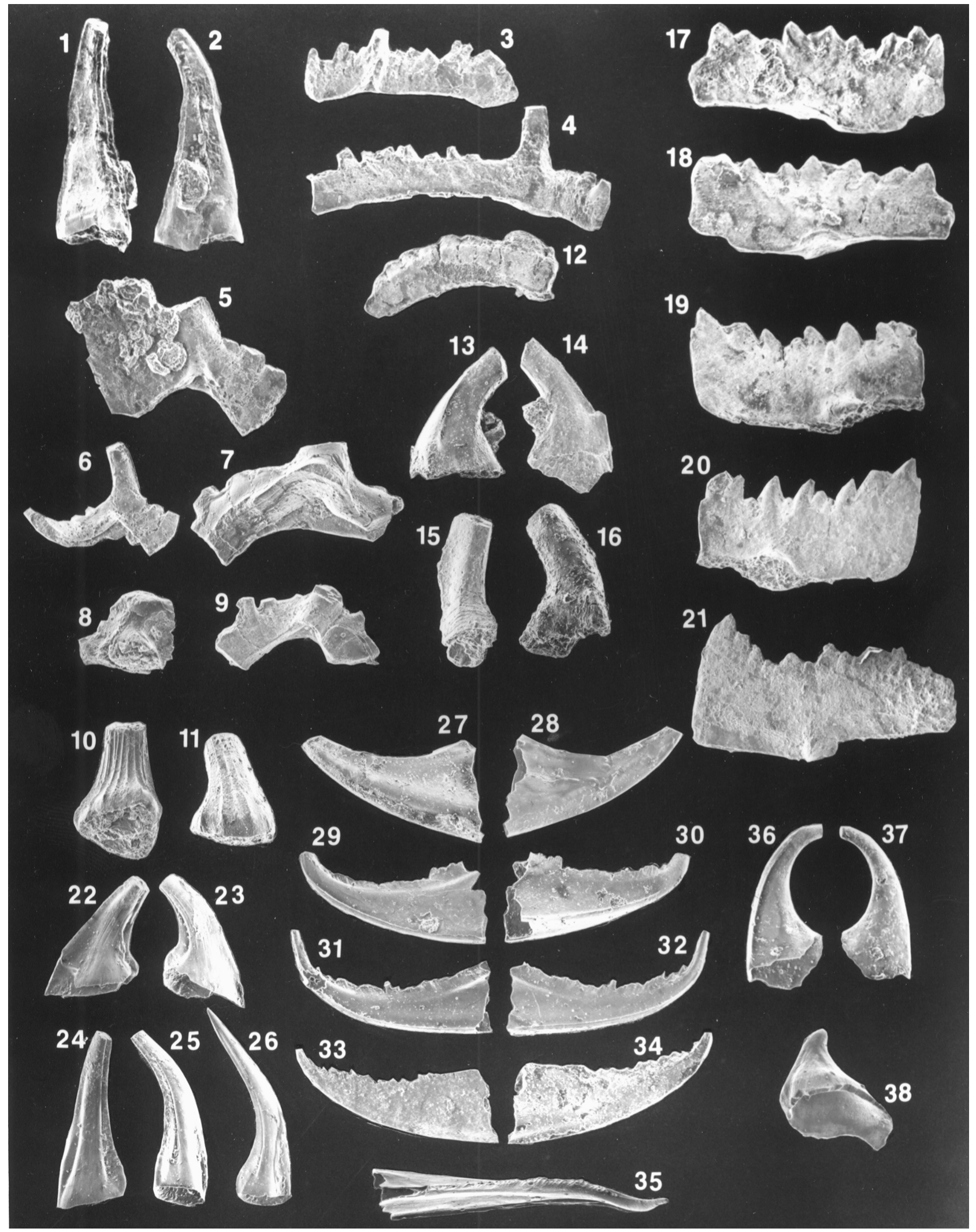




\section{Selected Series of U.S. Geological Survey Publications}

\section{Books and Other Publications}

Professional Papers report scientific data and interpretations of lasting scientific interest that cover all facets of USGS investigations and research.

Bulletins contain significant data and interpretations that are of lasting scientific interest but are generally more limited in scope or geographic coverage than Professional Papers.

Water-Supply Papers are comprehensive reports that present significant interpretive results of hydrologic investigations of wide interest to professional geologists, hydrologists, and engineers. The series covers investigations in all phases of hydrology, including hydrogeology, availability of water, quality of water, and use of water.

Circulars are reports of programmatic or scientific information of an ephemeral nature; many present important scientific information of wide popular interest. Circulars are distributed at no cost to the public.

Fact Sheets communicate a wide variety of timely information on USGS programs, projects, and research. They commonly address issues of public interest. Fact Sheets generally are two or four pages long and are distributed at no cost to the public.

Reports in the Digital Data Series (DDS) distribute large amounts of data through digital media, including compact discread-only memory (CD-ROM). They are high-quality, interpretive publications designed as self-contained packages for viewing and interpreting data and typically contain data sets, software to view the data, and explanatory text.

Water-Resources Investigations Reports are papers of an interpretive nature made available to the public outside the formal USGS publications series. Copies are produced on request (unlike formal USGS publications) and are also available for public inspection at depositories indicated in USGS catalogs.

Open-File Reports can consist of basic data, preliminary reports, and a wide range of scientific documents on USGS investigations. Open-File Reports are designed for fast release and are available for public consultation at depositories.

\section{Maps}

Geologic Quadrangle Maps (GQ's) are multicolor geologic maps on topographic bases in 7.5- or 15-minute quadrangle formats (scales mainly 1:24,000 or 1:62,500) showing bedrock, surficial, or engineering geology. Maps generally include brief texts; some maps include structure and columnar sections only.

Geophysical Investigations Maps (GP's) are on topographic or planimetric bases at various scales. They show results of geophysical investigations using gravity, magnetic, seismic, or radioactivity surveys, which provide data on subsurface structures that are of economic or geologic significance.
Miscellaneous Investigations Series Maps or Geologic Investigations Series (I's) are on planimetric or topographic bases at various scales; they present a wide variety of format and subject matter. The series also incudes 7.5-minute quadrangle photogeologic maps on planimetric bases and planetary maps.

\section{Information Periodicals}

Metal Industry Indicators (MII's) is a free monthly newsletter that analyzes and forecasts the economic health of five metal industries with composite leading and coincident indexes: primary metals, steel, copper, primary and secondary aluminum, and aluminum mill products.

Mineral Industry Surveys (MIS's) are free periodic statistical and economic reports designed to provide timely statistical data on production, distribution, stocks, and consumption of significant mineral commodities. The surveys are issued monthly, quarterly, annually, or at other regular intervals, depending on the need for current data. The MIS's are published by commodity as well as by State. A series of international MIS's is also available.

Published on an annual basis, Mineral Commodity Summaries is the earliest Government publication to furnish estimates covering nonfuel mineral industry data. Data sheets contain information on the domestic industry structure, Government programs, tariffs, and 5-year salient statistics for more than 90 individual minerals and materials.

The Minerals Yearbook discusses the performance of the worldwide minerals and materials industry during a calendar year, and it provides background information to assist in interpreting that performance. The Minerals Yearbook consists of three volumes. Volume I, Metals and Minerals, contains chapters about virtually all metallic and industrial mineral commodities important to the U.S. economy. Volume II, Area Reports: Domestic, contains a chapter on the minerals industry of each of the 50 States and Puerto Rico and the Administered Islands. Volume III, Area Reports: International, is published as four separate reports. These reports collectively contain the latest available mineral data on more than 190 foreign countries and discuss the importance of minerals to the economies of these nations and the United States.

\section{Permanent Catalogs}

"Publications of the U.S. Geological Survey, 1879-1961" and "Publications of the U.S. Geological Survey, 19621970" are available in paperback book form and as a set of microfiche.

"Publications of the U.S. Geological Survey, 1971-1981" is available in paperback book form (two volumes, publications listing and index) and as a set of microfiche.

Annual supplements for 1982, 1983, 1984, 1985, 1986, and subsequent years are available in paperback book form. 
